\title{
IONOSPHERIC GAS DYNAMICS OF SATELLITES AND DIAGNOSTIC PROBES
}

\author{
V. C. LIU \\ Dept. of Aerospace Engineering, \\ The University of Michigan, Ann Arbor, Mich., U.S.A.
}

(Received 3 February, 1969)

\begin{abstract}
The gas dynamics of interactions of a tenuous ionosphere with moving satellites and probes that have bearings on the diagnostics of the ionosphere are discussed. Emphasis is on the cases where the body is moving at mesothermal speeds, namely intermediate between the thermal speeds of ions and electrons of the ambient ionosphere. Methods of collision-free plasma kinetics with self-consistent field are used. The development of the topics for discussion starts with stationary Langmuir probe which entails the basic mechanism of body-plasma interaction that becomes further intricated as the body moves at a higher and higher speed. Applications of the theory of plasma interaction to meteors which move in the ionosphere are also presented.
\end{abstract}

\section{Introduction}

The study of charged particles and electric field near a conducting surface dated back at least half a century, known as the physics of gas discharge which is vital to the problems of lighting and vacuum tubes. A new aspect and stimulus have been added to the problem with the advent of the space flight, e.g., and electrically conducting satellite orbiting in the tenuous ionosphere of the earth. The fact that a body, such as a satellite, moves at a speed much higher than the ionic thermal speed of the ambient plasma, brings to bear a new gas dynamic phenomenon to the body-plasma interaction problem. The study of the flow field thus developed, which involves with the coupling between the charged particles and electric field, is herein called 'ionospheric gas dynamics', an interdisciplinary science of rarefied gas dynamics and electromagnetic fields. It has been known for some time that the disturbances of particle and field distributions near a moving body have important bearings upon its RADAR* detectibility and radio-frequency properties of antennas on-board. The use of artificial satellites and space rockets to carry instruments for the measure of the upper ionosphere has intensified the interest in the ionospheric gas dynamics. The disturbances present in the vicinity of a moving body must be properly taken into account in the planning and interpretation of various experiments made with on-board instruments. Besides the physics of body-plasma interactions are unique in themselves and are of considerable interest.

The gas-dynamic interference on the ionospheric measurements via diagnostic instruments on-board a satellite or other space vehicle can be illustrated with a cited example. When an electrostatic probe, often called Langmuir probe, which is installed on a satellite, is used to measure the electron density and temperature of the ambient

* Radio detecting and ranging. 
ionosphere, the plasma disturbances, which are created by the motion of the satellite and into which the probe is immersed, are actually being diagnosed. It is true that the properties of the disturbances which are probed are related to those of the ambient plasma of interest, proper interpretation of the probe data thus obtained must be made in order to eliminate the gas dynamic interference effect.

One of the alternatives to take this gas dynamic effect into account is to calibrate the probe with its attachment in an appropriately simulated plasma conditions in a laboratory setting. This is a well-established procedure in experimental gas dynamics. The laboratory simulation of space flight gas dynamics, however, has been very limited in success because of the inherent difficulty in proper scaling of the laboratory plasma parameters that can lead to meaningful calibration tests. One of such difficulties is to obtain a uniform, uncontaminated plasma of sufficient ionization level in the laboratory. In fact it has often been proposed to use the natural plasma of the upper ionosphere to carry out tests in the other areas of plasma studies. This hardship status in laboratory plasma has forced the prognostication of plasma phenomena to rely much on theoretical analysis to which the present review spends most of its pages.

It is intended, in the present review, to align the view points of ionospheric gas dynamics and diagnostics; to wit, the former is considered as a study of a response in particle and field distributions of a given source of disturbance which is imposed upon the ionosphere. Consequently a particular ionospheric diagnostics theory is merely an exercise of determining one or more of such responses from which the properties of the ambient plasma can be inferred. Keeping this in mind, we shall limit the discussion to those special configurations which are pertinent to the satellite probing of the ionosphere. The common features among various ionospheric gas dynamic problems should, however, be stressed, e.g., the study of the characteristics of a spherical electrostatic probe leads to considerable enlightening about the physics of plasma sheath of a spherical satellite.

There have been several comprehensive reviews in the related areas, e.g., concerning satellite disturbances $[1,2,3]$ and electrostatic probes $[4,5]$. In the last few years much development in experimental results and theories which have been achieved using self-consistent field approach. It is to the review of these new developments that the present paper is ascribed.

Prior to engage in the discussion of body-plasma interaction, it is necessary to be knowledgeable about the properties of the ionospheric medium and the microscopic accommodations of charged particles with solid surfaces that are pertinent to the ionospheric gas dynamics of interest.

\subsection{Physical properties of the ionosphere $[6,7,8]$}

The fact that the earth, along with its atmospheric envelope, is exposed to the solar radiation which contains sufficient energy at short wavelengths to cause appreciable photoionization of the earth's outer atmosphere explains the existence of the ionosphere. It refers loosely to the external, ionized layer of the atmosphere which is subdivided into regions: D, E, F and outer ionosphere in the order of increasing 
altitude and increasing fractional number density of electrons. The higher the altitude, the lower the atmospheric number density, hence the slower the rate of recombination, between free electrons and ions, which depends, among other factors, the collision frequencies of the atmospheric particles present. Since recombination reduces the electron concentration, this explains the altitude dependence of the degree of ionization level of the ionosphere. Inasmuch as the sun's radiation is the source of the ionization process, it is expected that the structure of the ionosphere must have diurnal variation and depends on the periodicity of maxima and minima of sunspot cycles. The dependence of electron density on the latitude of the earth is rather complex considering that geophysical influences must also be taken into account, and is not completely known. In view of these multiple factors of dependence, the designated layers of the ionosphere are not sharply distinct. The outer-most portion of the ionosphere, along with the earth's magnetic field, are determinated by the solar wind or interplanetary plasma.

It is important to describe the results of measurements of the principal ions in the upper ionosphere. It was found that near the lower boundary of the F-region, (160$700 \mathrm{~km}$ in altitude range), the principal ions present are $\mathrm{NO}^{+}$and $\mathrm{O}_{2}^{+}$; at higher altitude of the F-region; $\mathrm{N}^{+}$and $\mathrm{O}^{+}$which predominates. The outer ionosphere which extends from the top of the F-region until the boundary to the interplanetary space has been divided into sub-layers according to the principal ions present, e.g., $\mathrm{O}^{+}$is predominant up to an altitude of $900 \mathrm{~km} ; \mathrm{He}^{+}$, from 900 to $1900 \mathrm{~km} ; \mathrm{H}^{+}$ at higher altitudes.

Much of the information on the electron density at the extreme altitudes, such as several earth radii, are deduced from the measurements and inferences of the dispersion of radio whistlers which are low frequency $(1-30 \mathrm{Kc} / \mathrm{s})$ electromagnetic waves that are generated by lightning strokes and propagate along paths following the earth's magnetic field lines from one hemisphere to the other [9].

The upper ionosphere has been known to be in approximate quasi-equilibrium with unequal electron and ion temperatures, $T_{\mathrm{e}} \neq T_{\mathrm{i}}$, at least during the daytime. The bi-thermal nature of the ionosphere can be theorized in view of the mechanics of particle collision. It was stated earlier that the electrons in the ionosphere are heated as a result of photoionization of the ambient gas by solar ultraviolet radiation. Photoionization produces photoelectrons with energies of tens of electron volts, well in excess of the thermal energies of the other ionospheric constituents. The photoelectrons lose their excess energy as a result of inelastic collisions with the neutral molecules and elastic collisions with electrons and ions. On account of the mass-differential of the colliding partners, on which the energy exchange through collision depends, an electron, because of its small mass, loses very little kinetic energy when it collides with a heavy ion or neutral molecule. On the other hand, energy is exchanged readily in collisions with electrons (note that there are few electrons to share the excess energy from photoionization). Consequently the distribution of thermal speeds of the electrons is very close to the Maxwellian distribution with temperature $\left(T_{\mathrm{e}}\right)$ which is higher than the temperature of ions $\left(T_{\mathrm{i}}\right)$. The hot 
electron gas is cooled primarily by collisions with neutral particles at lower altitude, say below $250 \mathrm{~km}$, because of their relative abundance; by collisions with ions at higher altitudes because of the large collision cross-section of coulomb interaction. The ions act as a heat transfer agent and pass on the heat energy they extract from the electrons to the neutral gas for conduction toward lower altitudes [6].

On account of differences in the magnitudes of collision cross-sections between electrons and electrons, between electrons and ions, between ions and ions, etc., they are, accordingly, different mean free paths, $l_{\mathrm{e}, \mathrm{e}}, l_{\mathrm{e}, \mathrm{i}}, l_{\mathrm{i}, \mathrm{i}}$, etc.

For the purpose of quick reference, the estimated representative values of ionospheric parameters are given in Table I.

TABLE I

Typical values of ionospheric parameters

\begin{tabular}{llll}
\hline Altitude $(\mathrm{km})$ & 300 & 600 & 3000 \\
Electron Density $n_{\mathrm{e}}\left(\mathrm{cm}^{-3}\right)$ & $5 \times 10^{5}$ & $10^{5}$ & $2 \times 10^{3}$ \\
Mean free path $l_{i, n}(\mathrm{~cm})$ & $10^{5}$ & $10^{7}$ & $2 \times 10^{14}$ \\
$l_{\mathrm{e}, \mathrm{i}}(\mathrm{cm})$ & $10^{4}$ & $10^{5}$ & $10^{6}$ \\
Ionic Thermal Velocity $c_{\mathrm{i}}(\mathrm{Km} / \mathrm{s})$ & 1 & 1.5 & 6 \\
Electron Thermal Velocity $c_{\mathrm{e}}(\mathrm{Km} / \mathrm{s})$ & $2 \times 10^{2}$ & $3 \times 10^{2}$ & $4 \times 10^{2}$ \\
Debye length $\lambda_{\mathrm{D}}(\mathrm{cm})$ & 0.5 & 0.5 & 3 \\
Larmor Radius $L_{\mathrm{i}}(\mathrm{cm})$ & $5 \times 10^{2}$ & $8 \times 10^{2}$ & $10^{3}$ \\
$L_{\mathbf{e}}(\mathrm{cm})$ & 3 & 4 & 10 \\
Satellite Velocity $V_{\infty}(\mathrm{Km} / \mathrm{s})$ & 8 & 8 & 8 \\
\hline
\end{tabular}

Some conclusions pertinent to the ionospheric gas dynamics can be made in view of the tabulated ionospheric properties.

(1) Consider a body, having a characteristic size $L=1 \mathrm{~m}$, which moves in an ionosphere. It is obvious that any of the mean free paths at a listed altitude of the ionosphere is considerably larger than $L$. The state of a collision-free flow is therefore established provided the surface of the body is not concave; to be specific, the smallest mean free path $l_{\mathrm{e}, \mathrm{i}}$ is chosen as the characteristic one.

(2) Although the ionosphere is a heterogeneous medium, namely consisting particles of both neutral and charged species, each species, however, can be treated gas dynamically independent of the others in view of the collision-free state for ionospheric gas dynamics of interest here.

(3) When the orbiting speed $\left(V_{\infty}\right)$ of a satellite is mesothermal, namely $c_{\mathrm{i}} \ll V_{\infty} \ll c_{\mathrm{e}}$, where the ionic thermal speed $c_{\mathrm{i}}=\left(2 K T_{\mathrm{i}} / m_{\mathrm{i}}\right)^{1 / 2}$; electronic thermal speed $c_{\mathrm{e}}=$ $\left(2 K T_{\mathrm{e}} / m_{\mathrm{e}}\right)^{1 / 2}$, the gas dynamic effecton electrons is negligible except through their electrostatic coupling with the ions.

(4) Since the Larmor radius of ions $\left(L_{\mathrm{i}}\right)$ is usually much larger than the body size $L$, the earth's magnetic field effect on ionic flow can be neglected.

\subsection{Microscopic PARTICle ACCOMMOdation [10, 11]}

When a charged particle hits a solid surface, one of several alternatives may happen; 
to wit, it may be neutralized and then re-emitted as a neutral particle thus lost to the population of charged species it belongs, or reflect specularly and retains its charge, etc. With high kinetic energy of impact, an incident particle may cause secondary emissions from the surface. It is obvious that the statistical surface accommodations of the incident charged particles at the body would have important effect upon the distribution of particles near the body. Another consequence of this charge input by the incident particles is the resulting electric surface potential of the body. It is therefore important to understand this surface phenomenon in order to prescribe appropriately the boundary conditions of the plasma flow analysis.

The discussion of particle accommodation has been made difficult by the uncertainties of the actual surface conditions. Solid of crystalline material, to which many common solid such as metals belong, is of interest here. An ideal surface is one in which bulk properties of the material persists to the geometric surface. In a real surface which is generally prepared by mechanical working (grinding, machining, polishing, etc.), there is usually a surface layer, about 10 microns thick, which has been violently distorted. Hence the use of ideal solid surface model for particle accommodation must be exercised with caution.

Another property which distinguishes a real from an ideal surface is that the former generally suffers from presence of some sort of adsorbed gas layer. If a clean surface is placed in a gas, it is bombarded by gas particles, some of which stick to it. If they stick very tenaciously and virtually form a chemical compound with the surface atoms, they are said to be chemisorbed.

Chemisorption energies typically are of the same order as chemical reaction energies and ranges from 0.5 to 5 electron volts per atom. If the atoms are not strongly attached to the surface they are said to be physically adsorbed. Energies of physical adsorption are of the same order as latent heat of evaporation and ranges from 0.1 to 0.5 electron volts per atom. Physically adsorbed layers may be removed by moderate heating but chemisorbed layers require very high temperatures for their removal and may not be removed below the melting point in some cases. Adsorption behavior, from a kinetic point of view, is conveniently described by the sticking probability for molecules of the gas striking the surface. Sticking probabilities are usually in the range 0.1 to 1 for the first monolayer and then falls sharply as further layers are adsorbed. The amount of adsorption quickly reaches an equilibrium value which depends upon the partial pressure of the adsorbate and on the temperature, e.g., with a partial pressure of $10^{-7} \mathrm{~mm} \mathrm{Hg}$ of oxygen a clean metallic surface will acquire an oxide monolayer in about a minute.

Fortunately at the energy range of the typical incident particles of the ambient ionosphere on an orbiting satellite, this surface disparity is less consequential in ionospheric gas dynamics. Under the condition with surface potential of a few volts, the incident ions with positive charge are most probably neutralized by picking up an equivalent negative charge; an incident electron, absorbed. These are the accommodations which are used in the discussions here although most of the analyses can be modified to suit a different particle accommodation. 


\subsection{Equilibrium Surface potential without Photoemission $[12,13]$}

To illustrate the nature of the equilibrium surface potential $\left(\phi_{\mathrm{s}}\right)$ problem, a spherical metallic body of radius $R$ in a quiescent equilibrium plasma at temperature $T$ is considered. The incident flux of electrons which on the average out-race the ions will deposit a net amount of negative charges to the body assuming both species of particles are singly charged. The surface charges, being negative in polarity, tend to repel the electrons and attract the ions. This effect leads finally to an equilibrium state of the surface potential $\left(\phi_{\mathrm{s}}\right)$ such that the electronic flux balances the ionic flux in the charge input to the body. The equality of electronic and ionic fluxes can be expressed as

$$
-I_{\mathrm{i}}=\frac{1}{4} e n_{\mathrm{i}} A_{\mathrm{i}}\left(\frac{8 K T}{\pi m_{\mathrm{i}}}\right)^{1 / 2}=I_{\mathrm{e}}=\frac{1}{4} e n_{\mathrm{e}} A_{\mathrm{e}}\left(\frac{8 K T}{\pi m_{\mathrm{e}}}\right)^{1 / 2} \exp \left(-e \phi_{\mathrm{s}} / K T\right),
$$

where $n_{\mathrm{i}}, A_{\mathrm{i}}$ and $n_{\mathrm{e}}, A_{\mathrm{e}}$ denote the densities and effective collection areas for ions and electrons, respectively; $m_{\mathrm{i}}$ and $m_{\mathrm{e}}$, their respective masses. The effective collection area $A_{\mathrm{i}}$ may be a function of potential and the surface area of the body represents a lower limit on $A_{\mathrm{i}}$. It should be mentioned here that other sources of charge inputs, such as the photoemission of electrons from the body due to solar radiation, the intensive charge fluxes input if the body is in the radiation belt of the earth, etc., would change the equilibrium surface potential. The estimation of the equilibrium surface potential of a spherical body moving at mesothermal speeds, $c_{\mathrm{i}} \ll V_{\infty} \ll c_{\mathrm{e}}$, has been made by Brundin [13].

\subsection{Equilibrium SURfaCe POTENTIAL With PHOtoemission $[12,13]$}

When a satellite moves at higher altitude the charge input due to photoemission effect could become dominant. Again for the purpose of illustration, an extreme case is taken in which the photoemission effect is so strong relatively that the equilibrium surface potential $\left(\phi_{\mathrm{s}}\right)$ becomes positive relative to the ambient plasma. Under such condition the charge influx due to ionic thermal velocity becomes negligible. Consider the spherical body of radius $R$ which was described in Section 1.3. Here the equilibrium state is determined by the balance of electronic influx $I_{\mathrm{e}}$ due to thermal velocity of ambient electrons and the photoemission current $\left(I_{\mathrm{ph}}\right)$. The upper limit on the electronic influx, when no shielding is present, can be estimated as

$$
I_{\mathrm{e}}=\pi R^{2} e n_{\mathrm{e}}\left(\frac{8 K T}{\pi m_{\mathrm{e}}}\right)^{1 / 2}\left(1-\frac{e \phi_{\mathrm{s}}}{K T}\right) .
$$

The photoemission current depends on the magnitude of the positive surface potential $\left(\phi_{\mathrm{s}}\right)$ and the photoelectric work function of the body material $\left(\phi_{\mathrm{w}}\right)$. The balance equation for equilibrium (positive) surface potential can be written formally, assuming the photoelectric emission current $I_{\mathrm{ph}}$ at a given potential,

$$
e n_{\mathrm{e}}\left(\frac{8 K T}{\pi m_{\mathrm{e}}}\right)^{1 / 2}\left(1-\frac{e \phi_{\mathrm{s}}}{K T}\right)=\frac{I_{\mathrm{ph}}}{\pi R^{2}} \text {. }
$$


It is estimated that positive satellite potentials of more than a few volts is unlikely even in the interplanetary space. An accurate solution to the problem must involve a self-consistent analysis of the charge fluxes considering their dependence on surface potential.

\section{Methods of Collision-Free Plasma Flows}

The physical phenomena pertaining to the dynamic and electrostatic interaction between a moving body and a tenuous ionospheric medium constitute the scope of ionospheric gas dynamics. When the ratio of the characteristic mean free path $(l)$ to the size of the body $(L)$ is large, as in the case of a satellite in the upper ionosphere, the ionospheric flow relative to the body can be approximated as collision-free. This implies that the collisions, between the particles reflected from and incident on the body in question, have negligible effect on the particle distribution of the incident stream. The significance of this collisionless state is that in treating the body-plasma interaction, it is no longer suitable to treat the medium as a continuum due to rarity in collisions which are responsible for randomizing the particle motions and make the gas continuum-like. Instead, method of kinetic theory which is built on statistical particle dynamics should be used in order to obtain a better microscopic picture of the physical processes involved in the interaction.

The fact that the particles, which take part in the interaction, are of charged species adds considerable complications to the analysis particularly when the magnetic field effect is important. Fortunately in many of the ionospheric gas dynamic problems of interest, the geomagnetic field effect is almost negligible. An electrically conducing body which is immersed in a plasma will acquire an equilibrium surface potential $\left(\phi_{\mathrm{s}}\right)$ as shown in Section 1.3. The motion of the charged particles is now under the influence of the surface potential as well as the space charge potential which, in turn, depends on the motion of the charged particles themselves. It is this coupling of the field potential and the particles that gives the new complexion of the ionospheric gas dynamics. The development in a plasma of significant electric and possibly magnetic fields that exert a strong and frequently decisive influence on its motion is a fundamental feature of ionospheric gas dynamics, distinguishing it from the dynamics of neutral gases. It is noted that the presence of neutral particles can be essentially ignored in the ionospheric gas dynamic analyses. This stems from the fact that in the dynamics of the collisionless flows, the neutral and charged particles can be treated independently.

For simplicity an ionospheric medium consisting of singly charged ions and free electrons only is assumed. It is also postulated that upon collision with a surface, an electron will be absorbed; an ion, neutralized, hence becomes excluded from ion distribution in the flow field.

A body which is charged to a potential different from that of the ambient plasma maintains an electric field that attracts particles of opposite charge and repels those of like charge. The net charge density thus established in a region next to the body will be of opposite polarity to the net surface charges. It will shield the electric field 
of the body from penetrating into the undisturbed plasma which has ambient electron density $n_{\mathrm{e} \infty}$ and temperature $T_{\mathrm{e}}$. This region of charge imbalance is known as a plasma sheath. The thickness of the sheath is an index of the effectiveness of the plasma shielding effect and is defined as Debye shielding length $\left(\lambda_{\mathrm{D}}=\sqrt{\left.K T_{\mathrm{e}} / 4 \pi e^{2} n_{\infty}\right)}\right.$ when the body is stationary and its size is reduced to zero. The thickness of the sheath, which measures in the order of a few $\lambda_{\mathrm{D}}$, plays an important role in the bodyplasma interaction. It is observed from Table I on the typical values of ionospheric parameters that unless the body is unusually slender such as radio antenna, the shielding parameter $\left(\lambda_{\mathbf{D}} / L\right)$ is usually much less than unity. It will be seen that the smallness of the shielding parameter turns out to be a great asset in the simplifications of ionospheric gas dynamic analyses.

It is noted from the discussion in Section 1.3 that a typical satellite, which is not at a very high altitude where a dominant photoemission effect prevails, has a negative surface potential, $\phi_{\mathrm{s}}<0$, of a few volts. Another characteristic of a typical satellite orbiting in the ionosphere is its mesothermal speed which implies that the body velocity $\left(V_{\infty}\right)$ lies immediate between the thermal velocities of the ambient ions $\left(c_{\mathrm{i}}\right)$ and electrons $\left(c_{\mathrm{e}}\right)$, such that $c_{\mathrm{i}} \ll V_{\infty} \ll c_{\mathrm{e}}$ as evidenced in Table I. The body-plasma interaction, which is characterized by the conditions, $\phi_{\mathrm{s}}<0$ and $c_{\mathrm{i}} \ll V_{\infty} \ll c_{\mathrm{e}}$, is very prominent in ionospheric gas dynamics of satellites; they lead to a quasi-equilibrium distribution of the Maxwell-Boltzmann form for the electrons. This is, of course, not generally true with a diagnostic electrostatic probe to which both positive and negative potentials are applied.

\subsection{Governing EQUATIONS}

The statistical representation of particle distributions in a plasma field can be made at various levels of approximations, the appropriateness of which depend on the interaction potentials between the particles and the particle density [14]. In the study of ionospheric gas dynamics, it is found that the single-particle statistics expressed at the Boltzmann level of approximation is most appropriate. Following Boltzmann's description, a distribution function $f_{\alpha}(\mathbf{r}, \mathbf{c}, t)$ may be introduced which denotes, at time $t$, the phase density of the $\alpha$-species ( $\alpha$ refers to ions or electrons) at a point $(\mathbf{r}, \mathbf{c})$ in a six-dimensional phase space $(\mathbf{r}, \mathbf{c})$, known as the $\mu$-space. The statistical dynamics of the $\alpha$-particles, with mass $m_{\alpha}$, charge $Z_{\alpha} e$ in an electric field of potential $\phi(\mathbf{r})$, according to Boltzmann [15] is governed by

$$
\begin{aligned}
\frac{\partial f_{\alpha}}{\partial t}+\mathbf{c} \cdot \frac{\partial f_{\alpha}}{\partial \mathbf{r}}-\frac{Z_{\alpha} e}{m_{\alpha}} \frac{\partial \phi}{\partial \mathbf{r}} \cdot \frac{\partial f_{\alpha}}{\partial \mathbf{c}} & =\left(\frac{\delta f_{\alpha}}{\delta t}\right)_{\mathbf{c o l l} .} \\
& =\sum_{\beta} \iiint\left(f_{\alpha}^{\prime} f_{\beta}^{\prime}-f_{\alpha} f_{\beta}\right)\left|\mathbf{c}_{\beta}-\mathbf{c}\right| b \mathrm{~d} b \mathrm{~d} \varepsilon \mathrm{d}^{3} \mathbf{c}_{\beta}
\end{aligned}
$$

where $f_{\alpha}^{\prime} \equiv f_{\alpha}(\mathbf{r}, \mathbf{c}, t), f_{\beta} \equiv f_{\beta}\left(\mathbf{r}, \mathbf{c}_{\beta}, t\right)$ with subscript ' $\beta$ ' denoting the particle collision 
partner and superscript prime indicating quanty prior to a binary collision. The collision integral in Equation (3) expresses the rate of change of $f_{\alpha}(\mathbf{r}, \mathbf{c}, t)$ due to such collisions in which the velocities of the two colliding particles before collision are $\mathbf{c}^{\prime}$ and $\mathbf{c}_{\beta}^{\prime}$ and the final velocities are $\mathbf{c}$ and $\mathbf{c}_{\beta}$. The integration extends over all values of $\mathbf{c}_{\beta}$ and also over all values of the polar coordinates $b$ and $\varepsilon$ which specify the relative position of the two encountering particles. The terms on the left-hand side of Equations (3) express the total rate of change of $f_{\alpha}(\mathbf{r}, \mathbf{c}, t)$, when the particles stream in the $\mu$-space. The summation $\left(\sum_{\beta}\right)$ in front of the collision integral takes into account the collisions between various species in the medium.

The field potential $\phi(\mathbf{r}, t)$ is governed by the Poisson equation, when the magnetic field effect is neglected, with ions of charge $Z_{\mathbf{i}} e$ and free electrons,

$$
\nabla^{2} \phi=-4 \pi e\left[Z_{\mathrm{i}} n_{\mathrm{i}}(\mathbf{r}, t)-n_{\mathrm{e}}(\mathbf{r}, t)\right]
$$

The terms on the right hand side of Equation (4) denote the space charge effect on the field potential $\phi(\mathbf{r}, t)$. Equations (3) and (4) constitute a self-consistent system of plasma interaction without magnetic field effect. The above system governs not only interaction of plasma with body, waves with plasma and also waves with waves.

It is quite obvious that the simultaneous solution of the coupled non-linear Equations (3) and (4) is mathematically intractable. It will be shown that these equations with their full complexities preserved are not always necessary in treating many problems of interest particularly in the ionospheric gas dynamics of satellite. To illustrate, it is assumed that the surface potential $\phi_{\mathrm{s}}<0$, the body velocity $\left(V_{\infty}\right)$ is mesothermal, $c_{\mathrm{i}} \ll V_{\infty} \ll c_{\mathrm{e}}$, the plasma is neutral, $n_{\mathrm{i} \infty}=n_{\mathrm{e} \infty}=n_{\infty}$ and in thermal equilibrium, $T_{\mathrm{i}}=T_{\mathrm{e}}=T$. Consequently the electron distribution can be approximated by the Maxwell-Boltzmann law in terms of the field potential $\phi(\mathbf{r})$ in view of earlier discussion. The only unknowns left to be determined are the ion distribution function $f(\mathbf{r}, \mathbf{c}, t)$ and the field potential $\phi(\mathbf{r}, t)$.

\subsection{SimILARITY PARAMETERS}

Some physical insight into the Equations (3) and (4) can be gained by rewriting them in terms of dimensionless quantities. In defining these quantities, the following reference magnitudes as standards of comparison are introduced: Ionic thermal velocity $c_{\mathrm{i}}$ as characteristic velocity; body size $L$ (or wavelength as the case may be), macroscopic characteristic length; effective range of interaction between particles ${ }^{\dagger}$ $\sigma$, microscopic characteristic length; ambient ion density, $n_{\infty}$. The dimensionless representations (with superscript $*$ ) are as follows:

$$
\begin{aligned}
& t=\left(L / c_{\mathrm{i}}\right) \mathrm{t}^{*}, \quad \mathbf{r}=L \mathbf{r}^{*}, \quad \phi=(K T / e) \phi^{*} \\
& \mathbf{c}=c_{\mathrm{i}} \mathbf{c}^{*}, \quad b=\sigma b^{*}, \quad f=\left(n_{\infty} / c_{\mathrm{i}}^{3}\right) f^{*} \text {. }
\end{aligned}
$$

$\dagger$ A transverse distance from a scattering center that a scattered particle is deflected by an angle of $90^{\circ}$ or larger. 
In terms of the above representations, Equations (3) and (4) become, with $\alpha \equiv i$,

$$
\begin{aligned}
\frac{\partial f^{*}}{\partial t^{*}} & +\mathbf{c}^{*} \cdot \frac{\partial f^{*}}{\partial \mathbf{r}^{*}}-Z_{\mathrm{i}} \frac{\partial \phi^{*}}{\partial r^{*}} \cdot \frac{\partial f^{*}}{\partial \mathbf{c}^{*}}= \\
& =(\sqrt{2} \pi l / L)^{-1} \sum_{\beta} \iiint \int\left(f^{*^{\prime}} f_{\beta}^{* \prime}-f^{*} f_{\beta}^{*}\right)\left|\mathbf{c}_{\beta}^{*}-\mathbf{c}^{*}\right| b^{*} \mathrm{~d} b^{*} \mathrm{~d} \varepsilon d^{3} c_{\beta}^{*}
\end{aligned}
$$

where the characteristic mean free path $l=\left(\sqrt{2} \pi \sigma^{2} n_{\infty}\right)^{-1}$ and

$$
\left(\frac{\lambda_{\mathrm{D}}}{L}\right)^{2} \nabla^{2} \phi^{*}=e^{\phi^{*}}-n_{\mathrm{i}} / n_{\infty} .
$$

It is observed from Equations (5) and (6) that the dynamic and electrostatic similarity between different systems are governed by two ratios: Knudsen number, $l / L$ and shielding parameter, $\lambda_{\mathrm{D}} / L$. Let us investigate further the physical significance of these dimensionless equations. Consider Equation (5) which says, among other things, that when the condition $l / L \ll 1$ prevails, the collision-term exerts a dominant influence over the streaming-term. This implies that the flow field in question is collision-controlled and hence the particle distribution $f(\mathbf{r}, \mathbf{c}, t)$ is near locally Maxwellian. The significance of this conclusion can be better appreciated by considering the fact that non-trivial solutions to Equation (3) are rare; iterated solution is often sought to treat a physical problem. In view of the conclusion that when $l / L \ll 1$, collision-term dominates, the iteration must start with the locally Maxwellian distribution which is the steady state solution to Equation (3) when $l / L \rightarrow 0$ known as the zeroth order solution. It corresponds to the continuum equation for inviscidlike fluid. To iterate for the first order solution, the terms on the left-hand side of Equation (3) are prescribed in terms of the zeroth order solution. An intergral equation for $f(\mathbf{r}, \mathbf{c}, t)$ of the Fredholm type is thus obtained. Notice that the iteration procedure again is dictated by the collision-dominated condition. This constitutes essentially the approximation of Chapman-Enskog who solved the Boltzmann equation for transport phenomena of neutral gases [15].

The other limiting case, where $l / L \gg 1$, which represents collision-free or almost collision-free flows are of more interest to the ionospheric gas dynamics. A collisionfree flow is governed by Equations (3) or (5) with its collision term set to zero, and Equations (4) or (6). This is the approximation that will be followed in the present review of ionospheric gas dynamics. Should, however, a higher order approximation, an almost collision-free flow, is of interest for any reason, an iteration scheme can be obtained by prescribing the collision-term of Equation (3) in terms of collision-free solution* [16]. The equation, thus obtained, is a first order partial differential equation contrasting, in mathematical structure, to the equation that ChapmanEnskog use for the continuum flow.

* Which, in the calculation of sphere drag in flows of neutral particles [16] is a discontinuous function because of the molecular stream reflected from the body. 


\subsection{Asymptotic Solutions}

The dimensionless Poisson Equation (6) also provides some interesting approximations when the condition $\lambda_{\mathrm{D}} / L \ll 1$ prevails. The zeroth order approximation leads to the quasi-neutral flows where $n_{\mathrm{i}}(\mathbf{r})=n_{\mathrm{e}}(\mathbf{r})$. It describes a plasma state outside of the sheath (see Section 5) and the near wake (see Section 7).

Following Gurevich [17] who used Equation (6) to treat the near wake behind a spherical satellite, the wake is divided into two regions in one of which the ion density is not very small such that $\left(L / \lambda_{\mathrm{D}}\right)^{2} n_{\mathrm{i}}(\mathbf{r}) / n_{\infty}>1$; in the other of which the ion density is so small that $\left(L / \lambda_{\mathrm{D}}\right)^{2} n_{\mathrm{i}}(\mathbf{r}) / n_{\infty}<1$. The former refers to a region far away from the body; the latter, close to the body. The boundary separating them is defined by the condition

$$
\left(L / \lambda_{\mathrm{D}}\right)^{2} n_{\mathrm{i}}(\mathbf{r}) / n_{\infty}=1 .
$$

The field potential $\phi(\mathbf{r})$ in the far-region is approximated by the use of quasi-neutral condition $n_{\mathrm{i}}(\mathbf{r})=n_{\mathrm{e}}(\mathbf{r})=n_{\infty} \exp (e \phi / K T)$ which gives

$$
\phi(\mathbf{r})=-\frac{K T}{e} \ln \left(\frac{n_{\infty}}{n_{\mathbf{i}}(\mathbf{r})}\right) .
$$

The field potential $\phi(\mathbf{r})$ in the near-region is approximated by the use of

$$
\nabla^{2} \phi=0
$$

which is equivalent to assume that comparing with surface charge, the space charges are small in the region. The solution of Equation (9) for the near-region whose boundary is prescribed by Condition (7) and the surface potential at the body can be obtained. Gurevich's approximation, crude as it is, leads to much physical insight into the near-wake problem.

\subsection{WAVE MECHANICAL APPROACH}

An alternative approach to the mesothermal plasma interaction problem was proposed [18] which uses the Schroedinger equation to describe the collision-free plasma flows in lieu of the Boltzmann equation. The discussion will be limited to the steady motion and the absence of magnetic field although both restrictions can be removed. Consider a tenuous plasma, under equilibrium $n_{\mathrm{i} \infty}=n_{\mathrm{e} \infty}=n_{\infty}, T_{\mathrm{i}}=T_{\mathrm{e}}=T$, in which there is a negatively charged body moving at mesothermal speed $\left(V_{\infty}\right)$. In view of the prescribed body conditions stated above, the electron density $\left[n_{\mathrm{e}}(\mathbf{r})\right]$ can be approximated by the Maxwell-Boltzmann distribution

$$
n_{\mathrm{e}}=n_{\infty} \exp [e \phi(\mathbf{r}) / K T] .
$$

The Schroedinger wave equation for an ionic particle of mass $m_{\mathrm{i}}$ and charge $e$ in steady motion is [19]

$$
\hbar^{2} \nabla^{2} \psi(\mathbf{r})+2 m_{\mathrm{i}}[E-e \phi(\mathbf{r})] \psi(\mathbf{r})=0,
$$


where $\hbar=h / 2 \pi$ and $h$ denotes Planck's constant; $\phi(\mathbf{r})$ field potential; $E$, total energy of the particle in question. The wave function $\psi(\mathbf{r})$ has meaning such that $|\psi|^{2} \mathbf{d}^{3} r$ is the probability of finding a particle in volume element $\mathrm{d}^{3} r$ at $\mathbf{r}$. Thus if there are $n_{\infty}$ non-interacting identical particles per unit volume, $n_{\infty}|\psi|^{2}$ represents the probable number density at $\mathbf{r}$. Equation (11) can be used to describe ionic distribution in the field provided the particles are monoenergetic $(E)$ which is approximately true when the body velocity $V_{\infty} \gg c_{\mathrm{i}}$, the ionic thermal velocity.

It can be shown that for the ionic particles of interest in the plasma interaction, the de Broglie wavelength is negligibly small compared with the Debye shielding length over which $\phi(\mathbf{r})$ varies significantly. Hence a short wavelength approximation, known as WKBJ method in wave mechanics, can be introduced to Equation (11) for a simplified solution. In so doing all the diffraction effect which is important for the shadow (wake) zone of the flow field vanishes in this limit as might be expected. It is noted from geometric optics that if the coefficient of the $\psi$-term in Equation (11) were a constant, say $\alpha, \exp [i \alpha(\boldsymbol{\beta} \cdot \mathbf{r})]$ would be a solution to Equation (11), where $\boldsymbol{\beta}$ is a unit vector in the direction of wave propagation. This result suggests the following form for $\psi$

$$
\psi=A \exp [i W(\mathbf{r})]
$$

and if several rays pass through a point, we may write, instead of (12)

$$
\psi=\sum A \exp [i W(\mathbf{r})]
$$

the sum includes one term for each ray through the point. Equation (11), after the substitution of (12), becomes

$$
h^{2}\left[(\nabla W)^{2}-i \nabla^{2} W\right]=2 m_{\mathrm{i}}(E-e \phi)
$$

which reduces by short wave length approximation to

$$
\hbar^{2}(\nabla W)^{2}=2 m_{\mathrm{i}}(E-e \phi) .
$$

The first order partial differential Equation (14) for $W$ is equivalent to a system of ordinary differential equations for the rays (or orbits) of the particles. It is a form of Hamilton-Jacobi equation in classical mechanics. $W$ is proportional to the action. The possible orbits of ions are orthogonal to the surfaces of constant phase of the wave function $\psi$. The formal solution for $\psi$ can therefore be written in terms of $W$

$$
W(\mathbf{r})=\int \sqrt{2 m_{\mathrm{i}}} \hbar\{E-e \phi[\mathbf{r}(S)]\}^{1 / 2} \mathrm{~d} S,
$$

where $S$ is a parameter in terms of which the orbits of ions are described.

The advantage of the present method stems from the fact that the Schroedinger equation for $\psi$ is linear for a given $\phi(\mathbf{r})$. It turns out to be very helpful in obtaining a formal expression for the ion density which is needed to prescribe the space charge 
thus to prepare the Poisson equation for solution (see Section 5). It is admitted that the Hamilton-Jacobi equation rarely yields an exact solution. Nevertheless particular solutions with given initial conditions can often be obtained by numerical or graphical methods.

\section{Stationary Probe in a Quiescent Plasma}

In the present review of the self-consistent theory of ionospheric gas dynamics, we intend to develop the discussion from the simplest, physically significant case and evolve it to more complicated situations. From both the ionospheric diagnostics and gas dynamic view points, the electrostatic probe problem of Langmuir must be considered as an appropriate choice for the initial topic. The significance of Langmuir probe in plasma diagnostics is well known. It will be seen that the BernsteinRabinowitz theory of stationary probe [20] provides the foundation in both the methodology and the physical process of the ionospheric gas dynamics herein presented.

A method of diagnosing the properties of a tenuous plasma, suggested by Langmuir about fifty years ago, consists of measuring the electric current collected at a small wire, called probe, when different voltages that are biased both positive and negative relative to the ambient plasma are applied to the probe. This yields a voltage-current relation which characterizes the plasma under measurement. An electrostatic probe which maintains an electric field will attract particles of opposite charge and repel those of like charge. Thus a region of sheath to the probe will contain a net space charge to neutralize and shield the electric field of the probe from penetrating into the ambient plasma. This region of charge imbalance immediately outside of the probe surface is known as a plasma sheath in which the absolute value of the field potential decays from its probe value to almost nil at the outer edge of the sheath. A basic problem of probe theory is to determine the current collection at the probe of a prescribed surface potential $\left(\phi_{\mathrm{s}}\right)$ as a function of the ambient plasma parameters, e.g., electron density $\left(n_{\mathrm{e} \infty}\right)$ and temperature $\left(T_{\mathrm{e}}\right)$.

\subsection{A PROBE MODEL}

Consider a quiescent, fully ionized plasma of protons, each with charge $Z_{\mathrm{i}} e$ and mass $m_{\mathrm{i}}$; electrons, charge $Z_{\mathrm{e}} e$, and mass $m_{\mathrm{e}}$. It is assumed that the ambient plasma has equal electron and ion densities $\left(n_{\mathrm{i} \infty}=n_{\mathrm{e} \infty}=n_{\infty}\right)$ which is sufficiently hot and rarefied that the collision-free condition $(/ L \gg 1)$ is satisfied where $L$ denotes the probe size. The magnetic field effect is assumed negligible. We shall restrict the discussion to the time-independent case. The probe in question may be either a sphere or a twodimensional cylinder [21].

In view of the discussion in Section 2, we may write the self-consistent system of equations which govern the distributions of the distributions of charged particles and electric field in the sheath region as follows:

$$
\mathbf{c} \cdot \frac{\partial f_{\alpha}}{\partial \mathbf{r}}-\frac{Z_{\alpha} e}{m_{\alpha}} \frac{\partial \phi}{\partial \mathbf{r}} \cdot \frac{\partial f_{\alpha}}{\partial \mathbf{c}}=0
$$


where $\alpha$ denotes ions or electrons and $Z_{\mathbf{e}}=-1$

$$
\nabla^{2} \phi=-4 \pi e\left(Z_{\mathrm{e}} n_{\mathrm{e}}+Z_{\mathrm{i}} n_{\mathrm{i}}\right)=-4 \pi e\left[Z_{\mathrm{e}} \int f_{\mathrm{e}} \mathrm{d}^{3} c+Z_{\mathrm{i}} \int f_{\mathrm{i}} \mathrm{d}^{3} c\right] .
$$

The boundary values of the electric potential $\phi(\mathbf{r})$ and the particle distribution function $f_{\alpha}(\mathbf{r}, \mathbf{c})$ are prescribed accordingly as follows:

$$
\begin{aligned}
& \phi\left(R_{\mathrm{P}}\right)=\phi_{\mathrm{s}}, \quad \phi(\infty)=0 \\
& f_{\alpha}(\infty, \mathfrak{c}) \equiv f_{\alpha \infty}=n_{\infty}\left(m_{\alpha} / 2 \pi K T\right)^{d / 2} \exp \left(-m_{\alpha} c^{2} / 2 K T_{\alpha}\right),
\end{aligned}
$$

where $\alpha$ refers to the ion or electron species; $d=3$ for a sphere, or $d=2$ for a unit length of a cylinder.

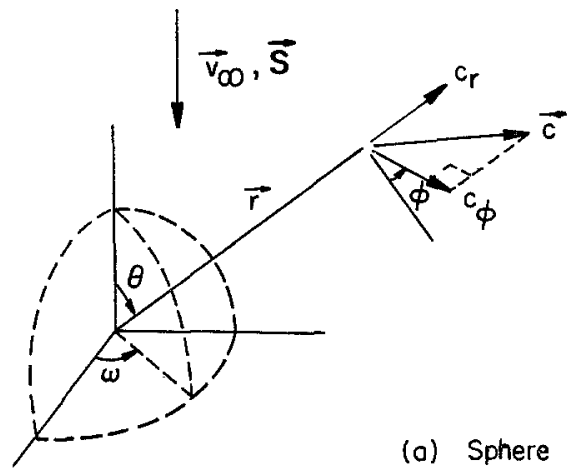

Fig. 1. Spherical coordinates (spherical probe).

So far the geometry of the probe has not been specified. To advance further, it is convenient to choose a coordinate system, fixed to the probe, which is appropriate to its configuration, e.g., use a spherical coordinate for a spherical probe (see Figure 1); cylindrical coordinate, cylindrical probe (see Figure 2). In the theory of probe, the particle densities $n_{\alpha}(\mathbf{r})$ and the current densities to the probe $I_{\alpha}$ are of primary interest

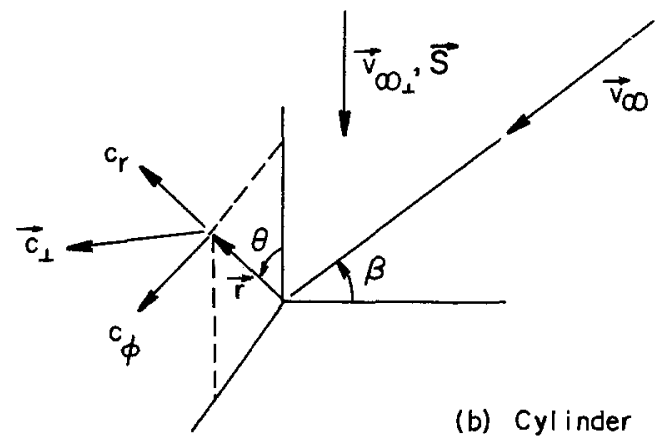

Fig. 2. Cylindrical coordinates (cylindrical probe). 
and can be expressed formally as follows:

$$
n_{\alpha}(\mathbf{r})=\int f_{\alpha}(\mathbf{r}, \mathbf{c}) \mathrm{d}^{3} c
$$

where the volume element in velocity space,

$$
d^{3} c=\left\{\begin{array}{l}
d c_{\mathrm{r}} c_{\phi} \mathrm{d} c_{\phi} \mathrm{d} \phi \\
\mathrm{d} c_{\mathrm{r}} d c_{\phi}
\end{array},\right.
$$

where the top line refers to a sphere; bottom line unit length of cylinder. And

$$
\left.I_{\alpha}\right|_{r=R_{\mathbf{P}}}=\int Z_{\alpha} e c_{\mathrm{r}} f_{\alpha}(\mathbf{r}, \mathbf{c}) \mathrm{d}^{3} c \mathrm{~d} S
$$

where

$$
\mathrm{d} S=\left\{\begin{array}{l}
r^{2} \sin \theta \mathrm{d} \theta \mathrm{d} w \\
r \mathrm{~d} \theta
\end{array},\right.
$$

where the top line refers to a sphere; bottom line, unit length of cylinder. The net current density $I_{\text {net }}=\left|I_{\mathrm{e}}\right|-I_{\mathrm{i}}$, where the electronic current is used as the reference.

\subsection{Method OF SOLUtion}

It is easily recognized in observing the coupled system of Equations (16) and (17) that, with a finite body $\left(R_{\mathrm{P}}>0\right)$ it is not possible to linearize the equations in order to apply the various methods of linear analysis. A usual procedure to unravel this non-linearly coupled system of Equations (16) and (17) is to construct first the formal solutions for $f_{\alpha}$ from Equations (16) and then substitute them into the Poisson equation (17) which is solvable for $\phi(\mathbf{r})$. It is observed that in stellar dynamics there is a theorem by Jeans which establishes the equivalence between an equation like (16) and the equation of motion of a particle and says that the most general solution of Equation (16) is an arbitrary function of the integrals of the equations of particle motion. We may reproduce Jeans theorem as follows [22]:

Consider a first order partial differential equation

$$
\partial f / \partial t+\mathbf{c} \cdot \partial f / \partial \mathbf{r}+\mathbf{F} \cdot \partial f / \partial \mathbf{c}=0
$$

for which the equations of characteristics are

$$
\mathbf{F}=\mathrm{d} \mathbf{c} / \mathrm{d} t, \quad \mathbf{c}=\mathrm{d} \mathbf{r} / \mathrm{d} t
$$

Let the solutions of Equation (25) be

$$
\mathbf{c}=\mathbf{c}\left(A_{1}, A_{2}, A_{3}, A_{4}, A_{5}, A_{6}, t\right) ; \quad \mathbf{r}=\mathbf{r}\left(A_{1}, A_{2}, \ldots, A_{6}, t\right),
$$

where the $A$ 's are the integration constants. The general solution to Equation (24) is expressible as an arbitrary function of $A$ 's.

$$
f(\mathbf{r}, \mathbf{c}, t)=f\left(A_{1}, A_{2} \ldots A_{6}\right) .
$$


The above assertion can be verified by the substitution of Equation (27) into Equation (24).

It should be noted that these integrals are not true constants but adiabatic invariants, thus the resulting solution for $f$ satisfies Equation (24) to within the accuracy of these invariants.

In view of Jeans theorem just described, the mathematical problem of searching for the solution of Equation (24) reduces to deciding the appropriate number of invariants needed for the problem in question and determining the functional forms of these invariants of interest.

It is further observed that with a symmetric body such as a sphere or a two-dimensional cylinder, in a quiescent plasma, the sheath must be symmetric with respect to the origin of the coordinate (r), hence the electric field of the sheath constitutes a central force system. It is known that the orbit of a particle in a central force field is two-dimensional and determined by two constants of the motion, the energy $E$, and the magnitude of the angular momentum $J$ [23] where

$$
E_{\alpha}=\frac{1}{2} m_{\alpha}\left(c_{\mathrm{r}}^{2}+c_{\phi}^{2}\right)+Z_{\alpha} e \phi,
$$

where $\alpha$ denotes ions or electrons.

$$
J_{\alpha}=m_{\alpha} c_{\phi} r .
$$

According to Jeans theorem, we can write the formal solutions to Equations (16).

$$
f_{\alpha}=f_{\alpha}\left(E_{\alpha}, J_{\alpha}\right) .
$$

The substitution of expressions (30) into Equation (17) leads to a non-linear integral-differential equation for $\phi(\mathbf{r})$ which will be solved by a method of iteration.

\subsection{TRANSFormation OF VARIABLeS}

In view of the above discussion it is obviously simpler to use $(E, J)$ instead of $(\mathbf{r}, \mathbf{c})$ as the phase variables to prescribe the orbits of collision-free particles in a central force sheath. The transformation of variables which are related by Equations (28) and (29) must be performed prior to the evaluations of integrals (20) and (22). Inasmuch as $E$ and $J$ are designated now as the phase variables, we shall drop their subscripts to follow the convention herein used.

Let us recapitulate the physical process in the collision-free plasma interaction in question. Consider the particles which move inbound toward the probe from the undisturbed plasma where they have Maxwellian distributions. Some of them, depending on their values of $E$ and $J$, may reach the surface of the probe $\left(r=R_{\mathrm{P}}\right)$ and be absorbed, if it is an electron and becomes neutralized, if an ion (class 1); a second alternative, for which a particle may have an orbit that has a turning point at $r_{\mathrm{T}}$ before reaching the probe, is designated class 2. There is still a third kind (class 3 ) which performs a bound orbit around the probe. It is clear that the distributions of the particles which determine the net space charge density in the sheath depend on how the particle orbits behave. It is also known that the $E, J$-values of a particle 
determine its orbit in a central force field much like the settings of the guidance for a sailing boat in a quiescent ocean. The mathematical problem, though intricate, is tractable. We shall illustrate this by following the change of their distribution functions which starts with

$$
f_{\alpha \infty}=n_{\infty}\left(m_{\alpha} / 2 \pi K T\right)^{d / 2} \exp \left(-E / K T_{\alpha}\right)
$$

where $d=3$ for sphere; $d=2$, unit length of a cylinder at ambient station $(r \rightarrow \infty)$. At a station $(r)$ closer to the probe, the local distribution function can be written

$$
f_{\alpha}=K_{\alpha}\left(E, J^{2}\right) f_{\alpha \infty}(E)
$$

where $K_{\alpha}\left(E, J^{2}\right)$ is a discontinuous function*:

(i) $K_{\alpha}=1$ for particles of class 1

(ii) $K_{\alpha}=2$ for particles of class 2 with $r \geqslant r_{\mathrm{T}}$. Note that this particle orbit forms mirror image relative to its radial turning point $r_{\mathrm{T}}$, hence it doubly contributes to the particle population at $r$ as compared to case (i).

(iii) $K_{\alpha}=0$ particles of class 2 with $r<r_{\mathrm{T}}$. It turns away before reaching station $\mathbf{r}$. It is noted that solution (31) satisfies Jeans theorem (30) and the boundary conditions as above specified. The function $K_{\alpha}\left(E, J^{2}\right)$ can be taken as a 'filter' which designates the degrees of availability for particles of specific $E$ and $J$ to contribute to the population of particles at the station.

The particle densities (20) and current densities (22), after the transformation of variables to $E$ and $J$ from the use of relations (28) and (29), become

$$
n_{\alpha}(r)=\int P_{\alpha}(r ; E, J) f_{\alpha \infty}(E) \mathrm{d} \Gamma
$$

and

$$
I_{\alpha}(r)=\left.\iint Z_{\alpha} c_{\mathrm{r}} P_{\alpha}(r ; E, J) f_{\alpha \infty}(E) \mathrm{d} \Gamma \mathrm{d} S\right|_{r=R_{\mathrm{P}}}
$$

where

$$
\mathrm{d} \Gamma=\left\{\begin{array}{l}
\mathrm{d} \phi \mathrm{d} E J \mathrm{~d} J \\
\mathrm{~d} E \mathrm{~d} J
\end{array}\right.
$$

where the top line refers to a sphere; the bottom line, to unit length of a cylinder. And

$$
P_{\alpha}(r ; E, J)=\frac{\left(m_{\alpha} r\right)^{1-d} K_{\alpha}\left(E, J^{2}\right)}{\left[2 m_{\alpha}\left(E-Z_{\alpha} e \phi\right)-J^{2} / r^{2}\right]^{1 / 2}} .
$$

The limits of integrals (32) and (33) cannot be specified until detailed orbital classifications are made.

* Possible bound orbits are discounted, the implication of which will be discussed later. 


\subsection{ORBITAL CLASSIFICATIONS}

The ultimate aim of an orbital classification for a given particle species in a given central force field, prescribed by a potential $\phi(r)$, is to identify on the $E, J^{2}$-plane limiting regions where the particles can reach. We shall follow the procedure of Bernstein and Rabinowitz. For illustration, consider ions in an attractive field. The convenient starting point is the energy integral for a particle in a central field $\phi(r)$,

$$
E-\left(Z_{\mathrm{i}} e \phi+J^{2} / 2 m_{\mathrm{i}} r^{2}\right)=\frac{1}{2} m_{\mathrm{i}} c_{r}^{2}
$$

obtained by combining relations (28) and (29). Equation (35) suggests that the centrifugal term contributes an equivalent repulsive potential. Accordingly an effective radial potential can be defined

$$
U(r, J)=Z_{\mathrm{i}} e \phi(r)+J^{2} / 2 m_{\mathrm{i}} r^{2}
$$

which governs the radial motion of a particle. To choose an interesting case we specify that the $r$-dependence of $\phi$ is such that $r^{2} \phi$ tends to zero as $r$ decreases, when $r$ is small; $r^{2} \phi=$ constant, when $r$ is large. Note that $U$, as a mathematical function of two variables $J$ and $r$ can be plotted as a function of $r$ for a given $J$. Some of the typical constant $J$ curves are shown in Figure 3 . The physical contents of the plot can be comprehended by remembering that the two terms on the R.H.S. of Equation (36) are opposite in signs; one is attractive; the other repulsive, they are competitive in their influences on the radial motion of a particle. That is why an orbit can have maximum $\left(U_{\max }\right)$ and minimum $\left(U_{\min }\right)$. A particle with angular momentum $J_{2}$ (see Figure 3) which experiences a potential well if the probe radius $R_{\mathrm{P}}$ is small enough not to extend to this region to interfere with it. Then there exist orbits which do not cut the probe, and which are of bounded radial variation, namely having bound orbits. One of these, e.g., has angular momentum $J_{2}$ (see Figure 3) and energy $E=U\left(a, J_{2}\right)=U\left(b, J_{2}\right)$ turns at $r=a$ and $r=b$.

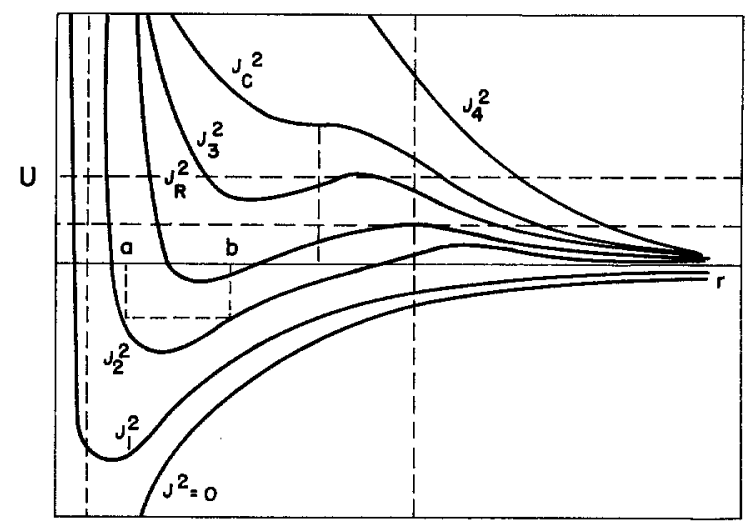

Fig. 3. Orbital classification diagram. Effective potential energy for radial motion vs. radius $U(r)[20]$. 
It is significant to locate the extrema of the $U(r)$ curves which is given by

$$
(\partial U / \partial r)_{J}=0 \quad \text { or } \quad J_{\mathrm{e}}^{2}=\left.m_{\mathrm{i}} r_{\mathrm{e}}^{3} Z_{\mathrm{i}} e(\mathrm{~d} \phi / \mathrm{d} r)\right|_{r=r_{\mathrm{e}}}
$$

which denotes the locus of extrema, $J_{c}\left(r_{\mathrm{c}}\right)$. Note that ions which have energy $E \geqslant U\left(r_{\mathrm{e}}, J\right)$ and angular momentum $J$ with $J^{2}>J_{\mathrm{e}}^{2}$ will reach the probe; $E<U\left(r_{\mathrm{e}}, J\right)$, $J^{2}>J_{\mathrm{e}}^{2}$, will turn away from the probe before reaching it. Furthermore, the ions with energy $E>U\left(r_{\mathrm{e}}, J\right)$ and with $J^{2}<J_{\mathrm{e}}^{2}$ will also be absorbed by the probe. Using the above criterion, a curve $J^{2}=G(E)$ in $E, J^{2}$-plane where

$$
\left.\begin{array}{rl}
J^{2} & =m_{\mathrm{i}} r^{3} Z_{\mathrm{i}} e \mathrm{~d} \phi / \mathrm{d} r \\
E & =Z_{\mathrm{i}} e \phi(r)+\frac{1}{2} Z_{\mathrm{i}} e r \mathrm{~d} \phi / \mathrm{d} r
\end{array}\right\} J^{2} \leqslant J_{\mathrm{e}}^{2}
$$

will define the limiting regions (see Figure 4) for the filtering function $K_{\mathrm{i}}\left(E, J^{2}\right)$.

\subsection{SOLUTIONS TO THE CURRENT COLLECTION}

The limits of integrations $(\mathrm{d} \Gamma$ ) for the particle densities (32) and current densities (33) can now be prescribed referring to Figure 4, namely, to integrate over regions, $C+D$, for $n_{\mathrm{i}}$ (3.17); region $\mathrm{D}$, for $I_{\mathrm{i}}(33)$ assuming that the probe radius $R_{\mathrm{p}}$ is large enough to allow only negligible bound orbits; the limits of integrations for $n_{\mathrm{e}}$ and $I_{\mathrm{e}}$ can be similarly prescribed (for details, see [21]), recall, however, electrons are in a repelling potential field if it is an attractive one for the ions as herein assumed.

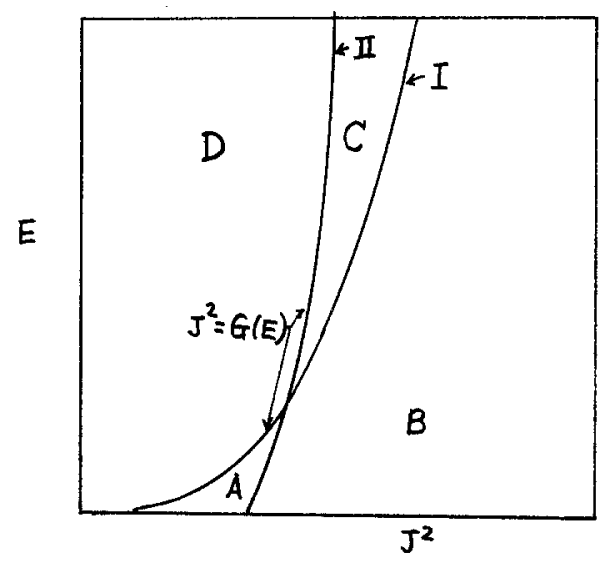

Fig. 4. $E, J^{2}$-phase plane [20]. I: $E=Z_{\mathrm{i}} e \phi(r)+J^{2} / 2 m_{\mathrm{i}} r^{2} ; \Pi: E=Z_{\mathrm{i}} e \phi_{\mathrm{s}}+J^{2} / 2 m_{\mathrm{i}} R_{\mathrm{P}^{2}}$.

With the inbound particles prescribed as Maxwellian distributions at the ambient station. The integrations over $\mathrm{d} \Gamma$ can be made over $\phi$ and $J^{2}$ only; the resultant particle densities and current densities are still definite integrals involving $\phi(r)$ which can be evaluated after $\phi(r)$ is prescribed. To complete the solution to the problem, 
the potential $\phi(r)$ which is governed by the Poisson Equation (17) must be determined. Equation (17), with the space charge terms, now expressed as definite integrals involving unknown $\phi(r)$, can be solved by using a special iteration procedure [21]. The iteration usually starts with an assumed net charge density and proceeds over large cycles of iterations until a self-consistent solution for $\phi(r)$ is obtained. The net current density $I_{\text {nct }}=\left|I_{\mathrm{e}}\right|-I_{\mathrm{i}}$ which represents the current collected at the probe can be integrated once the potential $\phi(r)$ is prescribed.

The numerical functional analysis herein described for both spherical and twodimensional cylindrical probes at different surface potentials $\phi_{\mathrm{s}}\left(\phi_{\mathrm{s}}<0\right.$ and $\left.\phi_{\mathrm{s}} \geqslant 0\right)$ and probe sizes, ignoring, however the presence of bound orbits, have been obtained by Laframboise [21]. Some of Laframboise's results are shown in Figures 5-10 and comparison with experiment [24] (see Figure 11).

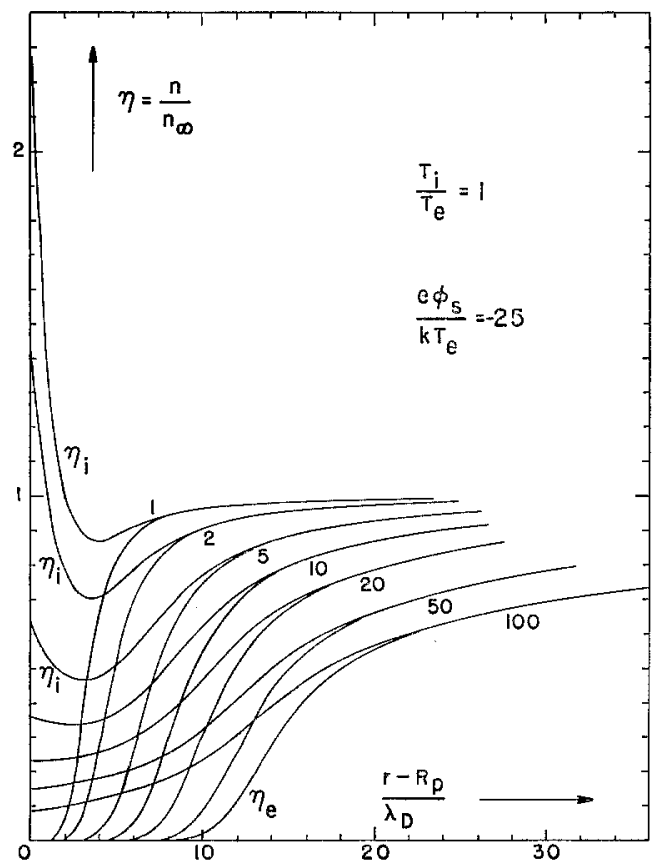

Fig. 5. Ion and electron charge densities vs. distance - spherical probe [21].

It is important to note that under a wide range of plasma states, the use of an isotropic monoenergetic distribution to approximate the ionic Maxwellian distribution at the ambient state leads to reasonable approximations for the probe currents. Bernstein and Rabinowitz used such an approximation for the case of a probe with high negative surface potential $\phi_{\mathrm{s}}[20]$. Extensive calculations were given by Chen [25]. Along the course of monoenergetic approximation, Lam [26] was able to carry out an asymptotic analysis of the Poisson equations for $\phi(r)$ in the limit $R_{\mathrm{P}} \gg \lambda_{\mathrm{D}}$, 
the Debye shielding length and gain analytically for the probe theory of Bernstein and Rabinowitz.

Enlightening comparisons of the contemporary theories of probe for diagnosis plasma under various conditions, including the theory of Allen, Boyd, and Reynolds [37] who used cold ion approximation and the earlier theory of Langmuir which is valid for very low densities and small probes has been given by Chen $[25,4]$. It is also significant to note a recent experiment by Chen, Etievant and Mosher [28] has shown excellent experimental verifications of Laframboise's analysis of probe currents. Further experimental works might be possible to gain insight into the validity of negligible bound orbit effect especially when the probe radius is small.

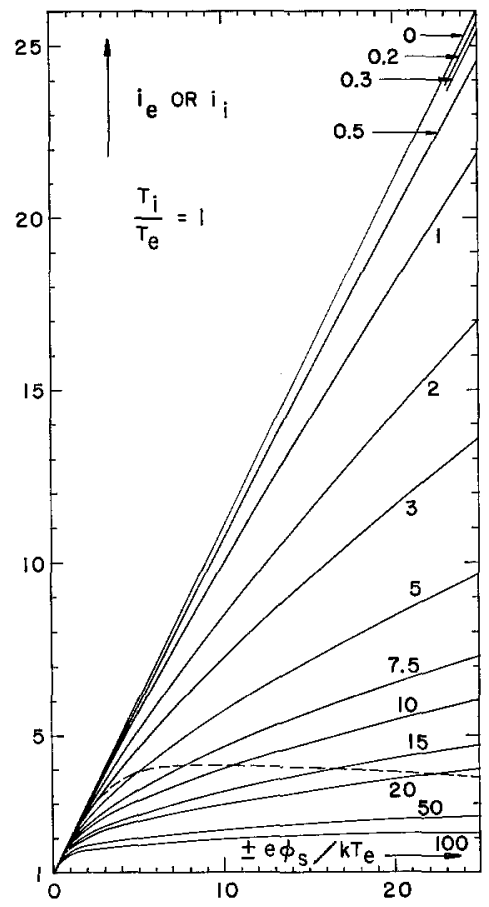

Fig. 6. Ion or electron current vs. probe potential - spherical probe [21].

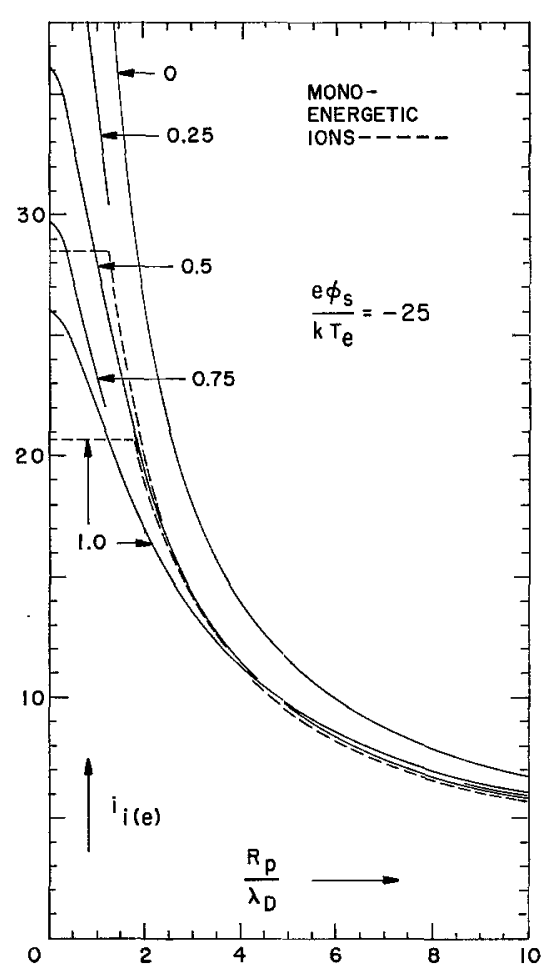

Fig. 7. Ion current vs. probe radius -spherical probe [21] $I_{i(e)}$ (referring to electron temperature).

As a concluding remark on the theory of probes in a quiescent plasma, it is perhaps significant to mention that the purpose of searching for an almost exact solution, such as Laframboise's, is not to merely gain a few percent of accuracy rather to comprehend the precise picture of plasma interaction at work. A further motivation for a study is to use it as a foundation to build the more involved plasma interactions of ionospheric gas dynamic interest (see Section 4). 


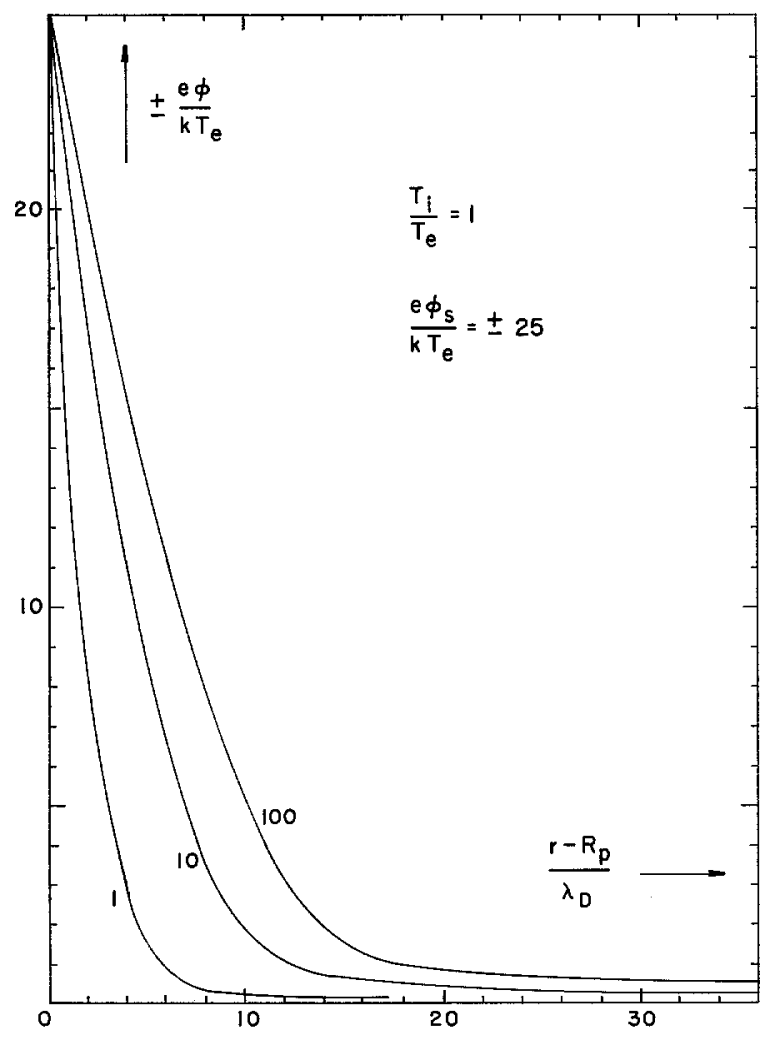

Fig. 8. Potential vs. distance - cylindrical probe [21].

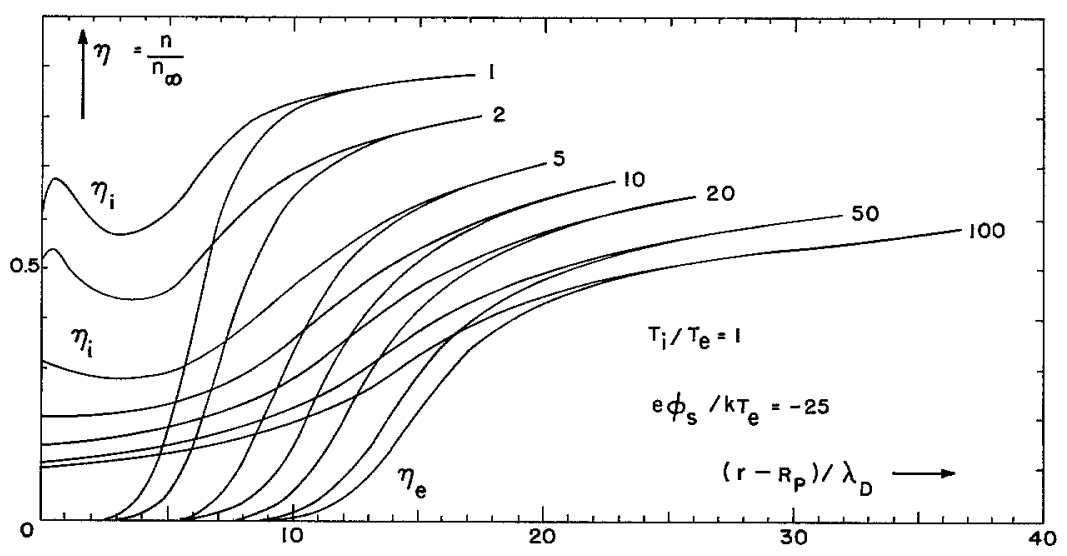

Fig. 9. Ion and electron charge densities vs. distance - cylindrical probe [21]. 


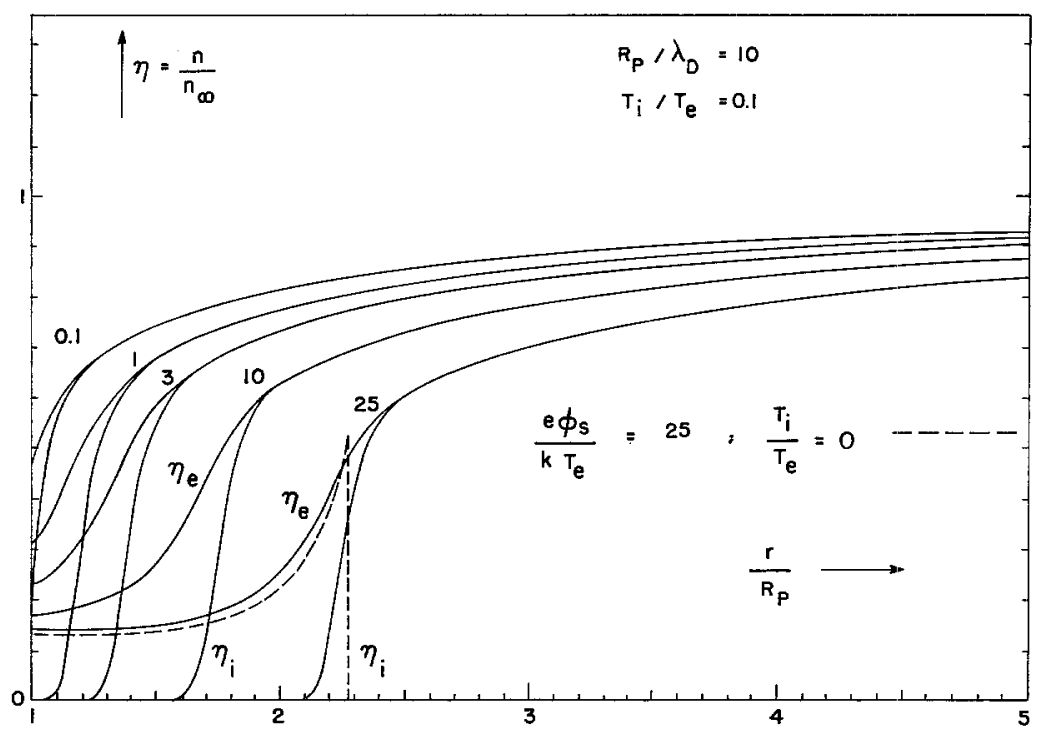

Fig. 10. Ion and electron charge densities vs. radius - electron-attracting cylindrical probe [21].

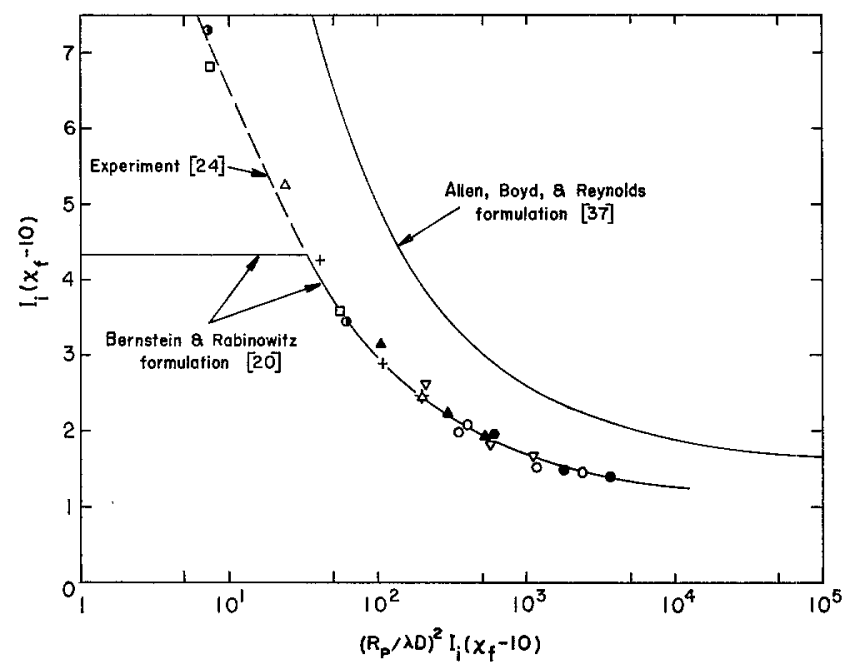

Fig. 11. Comparison between theory and measurements [21]. $T_{\mathrm{i}} / T_{\mathrm{e}} \ll 1, I_{\mathrm{i}} \rightarrow I_{\mathrm{i}(\mathrm{e})}$ [Figure 7]; $\chi=Z_{\mathrm{e}} \phi / K T$.

\section{Electrostatic Probe in Slowly Drifting Plasma}

The condition of a quiescent plasma relative to a diagnostic probe is rarely met either in laboratory plasma or in the ionosphere. In other words, a diagnosing probe is almost invariably exposed to a drifting plasma instead of a quiescent one. The superposition of a directed plasma motion toward a spherical or two-dimensional 
cylindrical probe will destroy the symmetry of the plasma sheath with respect to the probe. The electric field of the sheath will no longer be a central force system which has been the basic requirement for Bernstein-Rabinowitz' $E, J$-representation of particle orbits in the sheath. The transformation of variables to the $E, J^{2}$-plane has been the key of their success in formulating the functionals of probe theory.

It is intended to gain some physical insight of the drift effect without giving up completely the advantage of Bernstein-Rabinowitz approach. It is observed that a case can be made for a probe with a slowly drifting motion the velocity $\left(V_{\infty}\right)$ of which is small compared with the thermal velocity of ions $\left(c_{i}\right)$ in the ambient plasma. For instance, referring to the data on outer ionosphere where the ion temperature is high and the velocity of an orbiting satellite is much lower than if it is in lower altitude, the combination of these circumstances can lead to the condition, $V_{\infty} \ll c_{i}$, for a probe on-board. A somewhat similar case can be established for a probe in a laboratory plasma. Under this assumed condition, it is anticipated that the equipotential lines of the plasma sheath would deform slightly from their spherical (for a spherical stationary probe) into elliptical contours. Corresponding deviations for the other variables, such as particle densities, current densities, etc., from their respective stationary cases are also expected. A suitable perturbation parameter, preferably a dimensionless quantity, on which these deviations uniformly depend can be chosen. For instance, the speed ratio $\left(V_{\infty} / c_{\mathrm{i}}\right)$ apparently meets the requirement. The above discussion makes it possible to consider a perturbation analysis for the slow drift effect on the probe characteristics.

A proper treatment of the perturbation approach as such may involve with the perturbed characteristics in the (r, c) phase space relative to the characteristics defined by the Bernstein-Rabinowitz system. This indeed has been attempted with moderate success [29]. Although the transformation of variables to the $E, J^{2}$-plane helps, nevertheless the tracking of the orbits and their perturbations involves heavy accounting work in orbital classifications. Instead we intend to discuss an alternative approach to the plasma interaction problem, the wave-mechanical formulation using Schroedinger equation [18] which appears simpler mathematically in certain types of plasma interaction phenomena, e.g., the present one. The application of the wave mechanical approach to the probe problem in the discussion herein follows essentially the work of Chmielewski [30] which is an extension to the work [18]. The solution of interest is still left to be improved upon. Hence we would only bring out some features of the work for discussion.

\subsection{INTRODUCTION TO THE WAVE-MECHANICAL APPROACH}

The primary element of any perturbation analysis is the solution of the problem at zero perturbation, i.e., the zeroth order solution relative to which the perturbation at a finite value of the perturbation parameter, say $V_{\infty} / c_{\mathrm{i}}$, is made. Although we have such a zeroth order solution in Bernstein-Rabinowitz' stationary probe theory, it is however not expressed in wave mechanical formulation which is needed here. Accordingly as a preliminary step to attack the drift probe problem, a formulation 
of stationary probe in wave mechanical method is made. It provides an interesting comparison with the result of Section 3; the final results from the two different approaches agree as it is expected.

In the present section, we shall treat the same problem as postulated in Section 3.1. Instead of the collision-free Boltzmann equation (16), the motion of particles can be represented by the Schroedinger equation in wave mechanics (see Section 2.4). Consider a particle of energy $E$ in a central electrostatic field $\phi(r)$ it can be written

$$
\nabla^{2} \psi_{\alpha}+\frac{2 m_{\alpha}}{\hbar^{2}}\left[E-Z_{\alpha} e \phi(r)\right] \psi_{\alpha}=0,
$$

where $\hbar$ is related to Planck's constant $h=2 \pi \hbar$. The wave function $\psi_{\alpha}$ characterizes the motion of particle of mass $m_{\alpha}$. It is interpreted in a statistical sense such that $|\psi|^{2} \mathrm{~d}^{3} r$ represents the probability that the particle $(\alpha)$ will be found in the region between $\mathbf{r}$ and $\mathbf{r}+\mathrm{dr}$. Integration over the whole space thus yields a probability of unity, indicating that the particle is presented somewhere though its precise location is not necessarily known. In a more general sense, $\psi_{\alpha}$ can be considered as characterizing a stream of identical particles $(\alpha)$, then by appropriate normalization, $\left|\psi_{\alpha}\right|^{2}$ can be interpreted as the fractional number distribution of the stream. Although for convenience we continue referring to $\psi_{\alpha}$ in terms of single particle, this latter interpretation is the one of interest in the subsequent development.

\subsection{Spherical Potential Field}

Consider a spherically symmetric field $\phi(r)$. Let

$$
\psi_{\alpha}(r, \theta, w)=R_{\alpha}(r) Y_{\alpha}(\theta, w)
$$

and substitute into Equation (39). Separation of variables yields the equations:

$$
\begin{aligned}
& \frac{\mathrm{d}^{2}}{\mathrm{~d} r^{2}}\left(r R_{\alpha}\right)+\frac{2 m_{\alpha}}{\hbar^{2}}\left[E-Z_{\alpha} e \phi(r)-\frac{\lambda(\lambda+1) \hbar^{2}}{2 m_{\alpha} r^{2}}\right]\left(r R_{\alpha}\right)=0 \\
& \Lambda Y_{\alpha}=-\lambda(\lambda+1) Y_{\alpha},
\end{aligned}
$$

where

$$
A \equiv \frac{1}{\sin \theta} \frac{\partial}{\partial \theta} \sin \theta \frac{\partial}{\partial \theta}+\frac{1}{\sin ^{2} \theta} \frac{\partial^{2}}{\partial w^{2}} .
$$

There is an equivalence between the operator $A$ and the dynamic quantity $J^{2} / \hbar^{2}$ where $J$ is the total angular momentum and $\lambda(\lambda+1) \hbar^{2} \rightarrow J^{2}$ as $\hbar \rightarrow 0$. Their relation serves to link the discrete and continuous quantities which denote the angular momentum in wave mechanics and in classical mechanics, respectively. Hence, in the limit of short wave length approximation, $\hbar \rightarrow 0$, (see Section 2.6). Equation (41) may be rewritten

$$
\frac{\mathrm{d}^{2}}{\mathrm{~d} r^{2}}\left(r R_{\alpha}\right)+\frac{2 m_{\alpha}}{\hbar^{2}}\left[E-Z_{\alpha} e \phi(r)-\frac{J^{2}}{2 m_{\alpha} r^{2}}\right]\left(r R_{\alpha}\right)=0 .
$$


On account of spherical symmetry, this simple equation suffices to determine the particle motion. The solution of Equation (44) can be immediately written in view of the WKBJ approximation

$$
R_{\alpha}(r)=\frac{1}{m_{\alpha} r\left[Q_{\alpha}(r ; E, J)\right]^{1 / 2}} \exp \left[ \pm \frac{i}{\hbar} \int_{\infty}^{r} Q(r ; E, J) \mathrm{d} r\right]
$$

where

$$
Q_{\alpha}(r ; E, J)=\left\{2 m_{\alpha}\left[E-Z_{\alpha} e \phi(r)\right]-J^{2} / r^{2}\right\}^{1 / 2}
$$

Suppose that a stream of particles of species $\alpha$, each with energy $E$ and angular momentum $J$, moves inbound from the ambient station $(r \rightarrow \infty)$ towards the probe $\left(r=R_{\mathrm{P}}\right)$, the probability that a particle can be found in $\mathrm{d} r$ at $r$, where $R_{\mathrm{P}} \leqslant r<\infty$, is

$$
\left|R_{\alpha}(r)\right|^{2} \mathrm{~d} r=\frac{K_{\alpha}\left(E, J^{2}\right)}{\left(m_{\alpha} r\right)^{2} Q_{\alpha}(r ; E, J)} \mathrm{d} r .
$$

The factor $K_{\alpha}\left(E, J^{2}\right)$ has values 0,1 , or 2 as discussed in Section 3.3. It accounts for the two branches of a particle orbit associated with the possible choices of the algebraic sign in the exponential argument in Equation (45). These branches correspond to motions which approach ( - sign) and recede from $(+$ sign) the field center.

Note that the magnitude of the radial probability density $\left|R_{\alpha}(r)\right|^{2}$ would not be affected by any phase shift which amounts to multiplication factor $\exp i \varphi$ included in Equation (45). Observe particularly that $\left|R_{\alpha}(r)\right|^{2}$ is identical to the transition probability $P_{\alpha}$ in Equation (34) with $d=3$ for a spherical probe. Thus we recover the result of Bernstein and Rabinowitz for the spherical case.

\subsection{Cylindrical potential field}

With particles of species $\alpha$ in a cylindrically symmetric field $\phi(r)$, a similar development can be made restricting, however, the discussion to the motion in a transverse plane. Let

$$
\psi_{\alpha}(r, \theta)=R_{\alpha}(r) \Theta_{\alpha}(\theta)
$$

Equation (39), after the substitution of expression (48) leads to the separated system

$$
\begin{aligned}
\frac{\mathrm{d}^{2}}{\mathrm{~d} r^{2}}\left(\sqrt{r} R_{\alpha}\right)+\frac{2 m_{\alpha}}{\hbar^{2}}\left[E-Z_{\alpha} e \phi(r)-\frac{\lambda^{2} \hbar^{2}}{2 m_{\alpha} r^{2}}\right]\left(\sqrt{ } r R_{\alpha}\right) & =0 \\
\frac{\mathrm{d}^{2} \Theta}{\mathrm{d} \theta^{2}} & =-\lambda^{2} \Theta .
\end{aligned}
$$

In the present case, the equivalence $\mathrm{d}^{2} / \mathrm{d} \theta^{2} \sim-J^{2} / \hbar^{2}$ holds where $J\left(=J_{11}\right)$ is the angular momentum of the transverse motion of a particle. Again from Equation (50), 
in the limit $\hbar \rightarrow 0$, it follows that $\lambda^{2} \hbar^{2} \rightarrow J^{2}$ and Equation (49) takes the form

$$
\frac{\mathrm{d}^{2}}{\mathrm{~d} r^{2}}\left(\sqrt{r} R_{\alpha}\right)+\frac{2 m_{\alpha}}{\hbar^{2}}\left[E-Z_{\alpha} e \phi(r)-\frac{J^{2}}{2 m_{\alpha} r^{2}}\right]\left(\sqrt{r} R_{\alpha}\right)=0
$$

Once again by the use of WKBJ approximation, the solution to Equation (51) can be readily obtained from which the radial probability density of the $\alpha$ particles can be written

$$
\left|R_{\alpha}(r)\right|^{2}=\frac{K_{\alpha}\left(E, J^{2}\right)}{m_{\alpha} r Q_{\alpha}(r ; E, J)}
$$

where $Q_{\alpha}(r ; E, J)$ is defined as before namely function (46). It is observed again that the probability density (52) is identical to the transition probability $P_{\alpha}(r ; E, J)$ in Equation (34) for a cylindrical probe following the Bernstein-Rabinowitz formulation.

\subsection{Orbital STUdies}

The rest of the formulations leading to the particle densities and the current densities in the $E, J^{2}$-plane are essentially the same as in Section 3.3 except that with an absorption boundary at $R_{\mathrm{P}}$ for the reflected particles, $\psi_{\alpha}=0$. They are

$$
\begin{aligned}
& n_{\alpha}(r)=\iint f_{\alpha \infty}(E)\left|\psi_{\alpha}\right|^{2} d E \mathrm{~d} J^{d-1} \\
& I_{\alpha}(r)=\left.\iiint Z_{\alpha} e f_{\alpha \infty}(E) c_{\mathrm{r}}\left|\psi_{\alpha}\right|^{2} \mathrm{~d} E \mathrm{~d} J^{d-1} \mathrm{~d} S\right|_{r=R_{P}},
\end{aligned}
$$

where $d=3$ for a sphere; $d=2$ for unit length of a cylinder.

In addition to provide a zeroth order solution for the forthcoming perturbation analysis of the drift effect, the present formulation can throw some light on the bound orbit problem $[18,30]$, which appears important possibly when the plasma sheath is relatively thick. It is observed that when

$$
E-\left[Z_{\alpha} e \phi(r)+J^{2} / 2 m_{\alpha} r^{2}\right]>0
$$

which is the criterion in the orbital classification (Section 3.4), the solutions (45), under condition (55), are exponential in character. On the other hand, bound orbits can occur if

$$
E-\left[Z_{\alpha} e \phi(r)+J^{2} / 2 m_{\alpha} r^{2}\right]<0
$$

under which condition, the solutions to the Schroedinger equation (39), with the WKBJ approximation exhibit a harmonic behavior and are closely related to the problem of potential barrier penetration in wave mechanics. An analysis of the bound orbits effect in the light of potential barrier penetration appears feasible. In 
any event, additional hypothesis pertinent to the degree of 'fullness' of the potential wells associated with the bound orbits must be made.

\subsection{Perturbations of DRIFT EFFECT}

Consider a probe which has been described in Section 3.1. Superpose a directed velocity $V_{\infty}$ to the ambient plasma, given in Section 3.1, such that the distribution of the $\alpha$ particles in the ambient plasma becomes, referring to a reference frame fixed to the body,

$$
f_{\alpha \infty}=n_{\infty}\left(\frac{m_{\alpha}}{2 \pi K T}\right)^{d / 2} \exp \left[-\frac{m_{\alpha}}{2 K T_{\alpha}}\left(\mathbf{c}+V_{\infty}\right)^{2}\right]
$$

where $\alpha$ refers to ions or electrons; $d=3$ for a spherical probe or $d=2$ for a unit length of a cylinder. Notice that the positive streamwise axis points in the direction of body motion, and the particle velocity $\mathbf{c}$ is measured with respect to the body coordinates. It is assumed that $V_{\infty} / c_{\mathrm{i}} \ll 1$ where $c_{\mathbf{i}}$ denotes the thermal velocity of ions, $c_{\mathrm{i}}=\sqrt{2 K T_{\mathrm{i}} / m_{\mathrm{i}}}$.

It is observed that the displaced Maxwellian distribution (57) reduces to the quiescent case (19) when the drift velocity $V_{\infty}$ is set to zero. It is also evident that when $V_{\infty} / c_{\mathbf{i}} \ll 1$ as assumed, the resultant deviations in probe characteristics from their respective stationary case values are expected relatively small. This uniform dependence on a small parameter $V_{\infty} / c_{i}$ makes it fruitful to pursue a perturbation analysis for the slow drift effect on the probe characteristics. Although the perturbation procedure is straightforward [31,32], a judicious choice of functional expansions in terms of the perturbation parameter $\left(V_{\infty} / c_{\mathbf{i}}\right)$ often decides the fruitfulness of the analysis. Inasmuch as the state of the art on the problem of interest here [30] is still rudimentary, only a brief discussion of the formulation for a drift sphere is given here assuming $T_{\mathrm{i}}=T_{\mathrm{e}}=T$.

Let $\theta$ be the angle measured from the streamwise direction in a meridian plane of the sphere. Since $E=m_{\alpha} c^{2} / 2$ the distribution (57) can be rewritten [30]

$$
f_{\alpha \infty}(E)=n_{\infty}\left(\frac{m_{\alpha}}{2 \pi K T}\right)^{3 / 2} \exp \left\{-\frac{1}{K T}\left[E+V_{\infty} \sqrt{2 m_{\alpha} E} \cos \theta+m_{\alpha} V_{\infty}^{2} / 2\right]\right\}
$$

The plasma sheath of a drifting sphere tends to become elongated in the streamwise direction and the potential field is no longer perfectly radial. Particle energy remains a constant of motion but not the total angular momentum. The angular momentum component parallel to the drift direction remains constant as a result of axi-symmetry with respect to the streamwise axis. Sheath asymmetry is the consequence of nonisotrophy of the distribution at the ambient station and distortion of the collective charge distribution within the sheath. 


\subsection{Dimensionless equations}

To convert the Equations (39) and (17), which constitute the selfconsistent system here, we introduce the following dimensionless substitutions of the quantities (denoted by superscript primes)

$$
\begin{aligned}
& \psi^{\prime}=\left[\frac{K T\left(m_{\alpha} R_{\mathrm{P}}\right)^{2}}{c_{\alpha}}\right]^{1 / 2} \psi, \quad \chi=\frac{e \phi}{K T}, \quad \mathbf{r}^{\prime}=\frac{\mathbf{r}}{R_{\mathrm{P}}}, \\
& \hbar^{\prime}=\frac{\hbar}{m_{\alpha} R_{\mathrm{P}} c_{\alpha}}, \quad f_{\alpha \infty}^{\prime}=\frac{f_{\alpha \infty} c_{\alpha}^{3}}{n_{\infty}}, \quad n_{\alpha}^{\prime}=\frac{n_{\alpha}}{n_{\infty}}, \quad i_{\alpha}^{\prime}=\frac{I_{\alpha}}{I_{\mathrm{R} \alpha}}, \\
& c_{\mathrm{r}}^{\prime}=\frac{c_{\mathrm{r}}}{c_{\alpha}} \quad E^{\prime}=\frac{E}{K T} \quad j^{\prime}=\frac{J}{m_{\alpha} R_{\mathrm{P}} c_{\alpha}}, \quad \mathrm{d} S^{\prime}=\frac{\mathrm{d} S}{A_{\mathrm{P}}} \\
& \sigma=\left(\frac{R_{\mathrm{P}}}{\lambda_{\mathrm{D}}}\right)^{2}, \quad \quad \mu=\frac{m_{\mathrm{i}}}{m_{\mathrm{e}}}, \quad S_{\alpha}=\frac{V_{\infty}}{c_{\alpha}}, \quad A_{\mathrm{P}}=4 \pi R_{\mathrm{P}}^{2},
\end{aligned}
$$

where

$$
\lambda_{\mathrm{D}}=\left(\frac{K T}{4 \pi e^{2} n_{\infty}}\right)^{1 / 2}, \quad c_{\alpha}=\left(\frac{2 K T}{m_{\alpha}}\right)^{1 / 2}, \quad I_{\mathrm{R} \alpha}=\frac{Z_{\alpha} e n_{\infty} A_{\mathrm{p}} c_{\alpha}}{2 \sqrt{\pi}} .
$$

$I_{\mathrm{R} \alpha}$ denotes the random flux of species $\alpha$, incident upon a body at zero potential in a Maxwellian gas.

In terms of the above defined dimensionless quantities, the Equations (39) and (17) become

$$
\begin{aligned}
& \nabla^{2} \psi_{\alpha}^{\prime}+\left(1 / \hbar_{\alpha}^{\prime 2}\right)\left[E^{\prime}-Z_{\alpha} \chi\right] \psi_{\alpha}^{\prime}=0 \\
& \nabla^{2} \chi=\sigma\left(n_{\mathrm{e}}^{\prime}-n_{\mathfrak{i}}^{\prime}\right)
\end{aligned}
$$

particle densities

$$
n_{\alpha}^{\prime}(\mathbf{r})=\iint f_{\alpha \infty}^{\prime}\left|\psi_{\alpha}^{\prime}\right|^{2} \mathrm{~d} E^{\prime} \mathrm{d} J^{\prime 2}
$$

current densities

$$
i_{\alpha}=\left.2 \sqrt{\pi} \iiint f_{\alpha \infty}^{\prime} c_{\mathrm{r}}^{\prime}\left|\psi_{\alpha}^{\prime}\right|^{2} \mathrm{~d} E^{\prime} \mathrm{d} J^{\prime 2} \mathrm{~d} S^{\prime}\right|_{r^{\prime}=1},
$$

where

$$
f_{\alpha \infty}^{\prime}=\pi^{-3 / 2} \exp -\left[E+2 S_{\alpha} \sqrt{E} \cos \theta+S_{\alpha}^{2}\right]
$$

net current density

$$
i_{\text {net }}^{\prime}=i_{\mathrm{e}}^{\prime}-i_{\mathrm{i}}^{\prime} / \sqrt{\mu}
$$


particle energy

$$
E^{\prime}=c_{\mathrm{r}}^{\prime 2}+\frac{J^{\prime 2}}{r^{\prime 2}}+Z_{\alpha} \chi
$$

Boundary conditions:

$$
\begin{aligned}
& r=1\left\{\begin{array}{l}
\chi=\chi_{\mathbf{P}} \\
\psi_{\alpha}^{\prime}=0(\mathbf{c} \cdot \mathbf{r}>0)
\end{array}\right. \\
& r \rightarrow \infty\left\{\begin{array}{l}
\chi \rightarrow 0 \\
n_{\alpha}^{\prime} \rightarrow 1 .
\end{array}\right.
\end{aligned}
$$

\subsection{Perturbation expansions}

It is postulated that when a steady flow drift $\left(S_{\mathrm{i}} \ll 1\right)$ is superimposed on the ambient plasma, it impresses a uniform variation on all the sheath properties from their respective quiescent-state values. In terms of the perturbation parameter $S_{\alpha}$, we may perform the following expansions (superscripts primes denoting dimensionless are dropped hereafter)

where

$$
f_{\alpha \infty}=f_{\alpha 0}+S_{\alpha} f_{\alpha 1}+S_{\alpha}^{2} f_{\alpha 2}+\cdots
$$

$$
\begin{aligned}
f_{\alpha 0} & =\pi^{-3 / 2} \exp (-E) \\
f_{\alpha 1} & =-2 \sqrt{E} \cos f_{\alpha 0} \\
f_{\alpha 2} & =\left(2 E \cos ^{2} \theta-1\right) f_{\alpha 0} \\
\psi_{\alpha} & =\psi_{\alpha 0}+S_{\alpha} \psi_{\alpha 1}+S_{\alpha}^{2} f_{\alpha 2}+\cdots \\
n_{\alpha} & =n_{\alpha 0}+S_{\alpha} n_{\alpha 1}+S_{\alpha}^{2} n_{\alpha 2}+\cdots \\
i_{\alpha} & =i_{\alpha 0}+S_{\alpha} i_{\alpha 1}+S_{\alpha}^{2} i_{\alpha 2}+\cdots \\
\chi & =\chi_{0}+S_{\mathrm{i}} \chi_{1}+S_{\mathrm{i}}^{2} \chi_{2} \\
i_{\text {net }} & =i_{\text {net, } 0}+S_{\mathrm{i}} i_{\text {net, } 1}+S_{\mathrm{i}}^{2} i_{\text {net, },} .
\end{aligned}
$$

Note that $S_{\mathrm{i}}=\sqrt{\mu} S_{\mathrm{e}}$, hence $S_{\mathrm{i}} \gg S_{\mathrm{e}}$.

It is important to recall that the energy $E$ appearing in the equations for a drifting probe is measured relative to a reference frame fixed with the moving body (Sections 4.1 and 4.5 ). On the other hand, with a stationary probe, call it $E_{0}$, it refers to particle energy measured with respect to the earthfixed coordinates. Accordingly, $E=E_{0}+$ $+\Delta E\left(S_{\alpha}, \theta\right)$ where $\Delta E$ represents an energy change associated with the drifting motion. The dependence on $\theta$ appears because the energy change differs for particles approaching from different indurations relative to the streamwise direction; $\Delta E$ denotes an energy increment for a particle approaching from the upstream direction and a decrement for one entering the sheath from the downstream. Since $S_{\alpha} \ll 1$, these changes, when summed over all incoming particles, will approximately cancel and are therefore neglected. 


\subsection{ZEROTH-ORDER EQUATIONS}

With the substitution of the expanded functions (70) and (72) into Equations* (59) to (69), the boundary value problem for the perturbed sheath breaks down into a sequence of boundary value systems for terms of successive order in the expansions. The zeroth order system becomes

$$
\begin{aligned}
& h_{\alpha}^{2} \nabla^{2} \psi_{\alpha 0}+\left[E-Z_{\alpha} \chi_{0}(r)\right] \psi_{\alpha 0}=0 \\
& n_{\alpha 0}=\iint f_{\alpha 0}\left|\psi_{\alpha, 0}\right|^{2} \mathrm{~d} E \mathrm{~d} J^{2} \\
& \nabla^{2} \chi_{0}=\sigma\left(n_{\mathrm{e} 0}-n_{\mathrm{i} 0}\right)
\end{aligned}
$$

at $r=1$

$$
\begin{aligned}
\chi_{0} & =\chi_{\mathbf{P}} \\
\psi_{\alpha 0} & =0 \quad(\mathbf{c} \cdot \mathbf{r}>0)
\end{aligned}
$$

at $r \rightarrow \infty$

$$
\begin{aligned}
& \chi_{0} \rightarrow 0 \\
& n_{\alpha 0} \rightarrow 1 \\
& i_{\alpha 0}=2 \sqrt{\pi}\left[\iiint f_{\alpha 0} c_{\mathrm{r}}\left|\psi_{\alpha 0}\right|^{2} \mathrm{~d} E \mathrm{~d} J^{2} \mathrm{~d} S\right]_{r=1} \\
& i_{\text {net }, 0}=i_{\mathrm{e} 0}-i_{\mathrm{i} 0} / \sqrt{\mu} .
\end{aligned}
$$

\subsection{HIGHER ORDER SYSTEMS}

The first order equations are

$$
\hbar_{\alpha}^{2} \nabla^{2} \psi_{\alpha 1}+\left[E-Z_{\alpha} \chi_{0}(r)\right] \psi_{\alpha 1}=a_{1} \chi_{1} \psi_{\alpha 0}
$$

where $a_{1}=Z_{\alpha}$ for ions and $a_{1}=Z_{\alpha} / \sqrt{\mu}$ for electrons

$$
\begin{aligned}
n_{\alpha 1}(\mathbf{r}) & =\iint\left[f_{\alpha 0}\left(\psi_{\alpha 0}^{*} \psi_{\alpha 1}+\psi_{\alpha 1}^{*} \psi_{\alpha 0}\right)+f_{\alpha 1}\left|\psi_{\alpha 0}\right|^{2}\right] \mathrm{d} E \mathrm{~d} J^{2} \\
\nabla^{2} \chi_{1} & =\sigma\left[\left(n_{\mathrm{e} 1} / \sqrt{\mu}\right)-n_{\mathrm{i} 1}\right]
\end{aligned}
$$

at $r=1$

$$
\begin{aligned}
\chi_{1} & =0 \\
\psi_{\alpha 1} & =0 \quad(\mathbf{c} \cdot \mathbf{r}>0)
\end{aligned}
$$

* The subscript primes denoting dimensionless are dropped. 
at $r \rightarrow \infty$

$$
\begin{aligned}
\chi_{1} \rightarrow 0 \\
n_{\alpha 1} \rightarrow 0 \\
i_{\alpha 1}=2 \sqrt{\pi}\left[\iiint f_{\alpha 0}\left(\psi_{\alpha 0}^{*} \psi_{\alpha 1}+\psi_{\alpha 1}^{*} \psi_{\alpha 0}\right)+\left.f_{\alpha 1}\left|\psi_{\alpha 0}\right|^{2} c_{\mathrm{r}} \mathrm{d} E \mathrm{~d} J^{2}\right|_{r=1}\right. \\
i_{\text {net } 1}=\left(i_{\mathrm{e} 1}-i_{\mathrm{i} 1}\right) / \sqrt{\mu}
\end{aligned}
$$

where superscript $*$ denotes complex conjugate. It is noted that the first order solution can be obtained only after the zeroth order solution is given.

The second order equations are

$$
\hbar_{\alpha}^{2} \nabla^{2} \psi_{\alpha 2}+\left[E-Z_{\alpha} \chi_{0}(r)\right] \psi_{\alpha 2}=a_{1} \chi_{1} \psi_{\alpha 1}+a_{2} \chi_{2} \psi_{\alpha 0}
$$

where $a_{2}=Z_{\alpha}$ for ions and $a_{1}=Z_{\alpha} / \mu$ for electrons

$$
\begin{aligned}
n_{\alpha 2}(\mathbf{r})=\iint\left[f_{\alpha 0}\left(\psi_{\alpha 0}^{*} \psi_{\alpha 2}+\psi_{\alpha 2}^{*} \psi_{\alpha 0}+\left|\psi_{\alpha 1}\right|^{2}\right)\right. \\
\left.+f_{\alpha 1}\left(\psi_{\alpha 0}^{*} \psi_{\alpha 1}+\psi_{\alpha 1}^{*} \psi_{\alpha 0}\right)+f_{\alpha 2}\left|\psi_{\alpha 0}\right|^{2}\right] \mathrm{d} E \mathrm{~d} J^{2} \\
\nabla^{2} \chi_{2}=\sigma\left[\left(n_{\mathrm{e} 2} / \mu\right)-n_{\mathrm{i} 2}\right]
\end{aligned}
$$

at $r=1$

$$
\begin{aligned}
\chi_{2} & =0 \\
\psi_{\alpha 2} & =0 \quad(\mathbf{c} \cdot \mathbf{r}>0)
\end{aligned}
$$

at $r \rightarrow \infty$

$$
\begin{aligned}
& \chi_{2} \rightarrow 0 \\
& n_{\alpha 2} \rightarrow 0 \\
& i_{\alpha 2}=2 \sqrt{\pi}\left\{\int \int \int \left[f_{\alpha 0}\left(\psi_{\alpha 0}^{*} \psi_{\alpha 2}+\psi_{\alpha 2}^{*} \psi_{\alpha 0}+\left|\psi_{\alpha 1}\right|^{2}\right)\right.\right. \\
& \left.+f_{1}\left(\psi_{\alpha 0}^{*} \psi_{\alpha 1}+\psi_{\alpha 1}^{*} \psi_{\alpha 0}\right)+f_{\alpha 2}\left|\psi_{\alpha 0}\right|^{2}\right]\left.c_{\mathrm{r}} \mathrm{d} E \mathrm{~d} J^{2}\right|_{r=1} \\
& i_{\text {net } 2}=\mu^{-1}\left[i_{\mathrm{e} 2}-i_{\mathrm{i} 2} / \sqrt{\mu}\right] \text {. }
\end{aligned}
$$

Notice again that the second order equations are prescribed in terms of the zeroth and first order solutions.

4.10. SOLUTIONS to DRIFTING PROBE EQUATIONS

The equations developed in Section 4.9 for an electrostatic probe in a slowly drifting, Maxwellian plasma have been numerically integrated by Chmielewski [30]. Notice that the drift-induced corrections to sheath characteristics are expressed in terms of 
corresponding solutions for a stationary probe. The stationary probe solutions of Laframboise [21] were used to determine the configuration of the locus of extrema in the $E, J^{2}$-plane as a preliminary part of each calculation. Extensive calculations of the first and second order solutions are available [30], only a few samples will be shown here for illustration (see Figures 12,13). In view of the nature of approximations [30], the results are taken as a gross estimation of the drift effects.

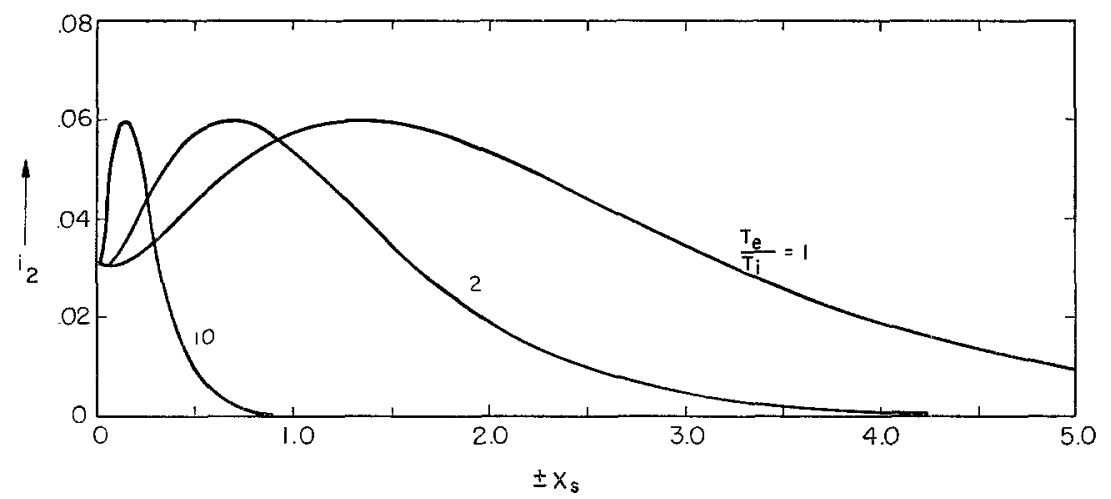

Fig. 12. Second order current (i2) vs. probe potential $\left(\phi_{\mathrm{s}}\right)$ - repelling sphere [30].

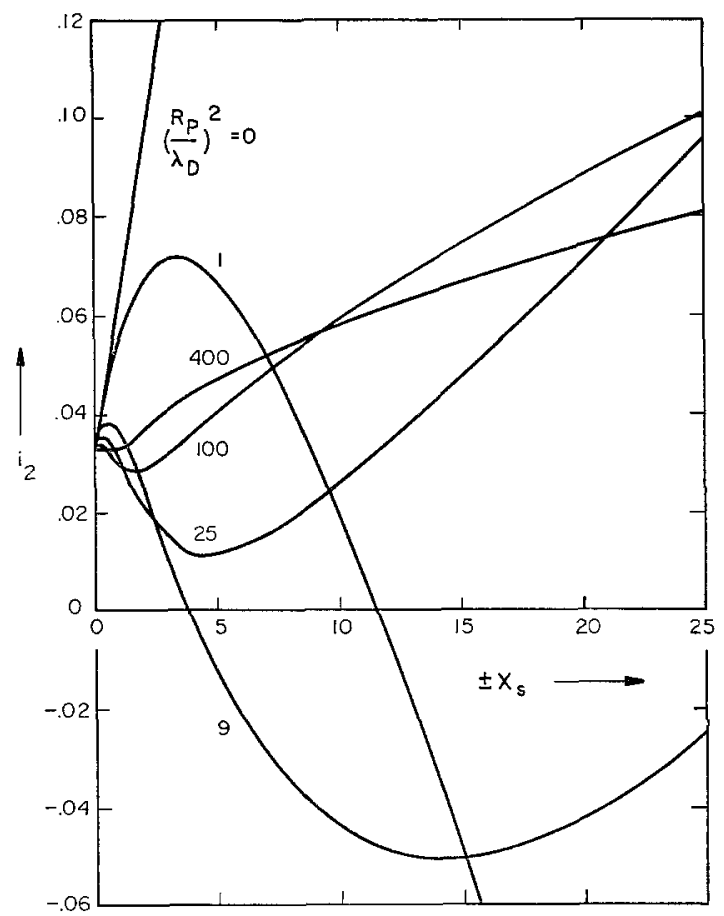

Fig. 13. Second order current $\left(i_{2}\right)$ vs. probe potential $\left(\phi_{\mathrm{s}}\right)-$ attracting sphere [30]. 
Relatively few experimental results which displays the effect of drift on current collection by an electrostatic probe are available. Some works have been reported [24], [33] in which cylindrical probes oriented at different angles relative to directed plasma were used. Their findings verify that drift effects are most readily observable in the ion saturation region. However, any quantitative comparison of the theoretical results [30] and [24] and [33] are unwarrantable because the experiments were not operated with collisionless ionic flows.

In conclusion, it appears that only a small effect on the stationary probe characteristics is expected when a slow plasma drift is imposed. The principal change occurs in the ion saturation region of the current-voltage characteristic curve of the probe. In general, the change in the net current indicated by the theoretical results are much smaller than those predicted by the contemporary theories with ad hoc approximations. The above conclusion can be reconciled with the observation that the electrostatic field effect on the sheath structure is large compared with a small flow perturbation plus the fact that the local current perturbations tend to cancel between the upstream and downstream sides of the probe when the average (over the probe surface) for current is taken. For instance the first order correction for the net current vanishes. Nevertheless sample calculations for a spherical probe does indicate that the effect of drift on the field potential distribution is to steepen the sheath potential on the upstream side of the probe; to make it decay more gradually on the downstream side. Far from the probe, the decay of field perturbation appears to be more gradual at ion-attracting potentials than at electronattracting potentials.

\section{Ionospheric Flows at Mesothermal Speeds. I: Sheath and Quasi-Neutral Flows}

When a satellite moves in the upper ionosphere, the typical body velocity $\left(V_{\infty}\right)$ relative to the ambient medium is mesothermal, namely it is much larger than the thermal velocity of the ions $\left(c_{\mathrm{i}}\right)$ much smaller than that of the electrons $\left(c_{\mathrm{e}}\right)$, $c_{\mathrm{i}} \ll V_{\infty} \ll c_{\mathrm{e}}$. The gas dynamic aspect of such a body-plasma interaction has a unique feature: While the flow of the ion species has hyperthermal characteristics, that of the electron species is low subthermal, in fact, quasi-stationary. The ion and electron species are of course coupled, through an electrostatic field. In other words, the gas dynamic effect of the flow is initially imparted to the distribution of the ion species and transmitted to that of the electron species via the electrostatic coupling.

The extent of plasma disturbances in the neighborhood of a body facing a mesothermal stream can be conveniently divided into the following zones: (I) Sheath and its transition to quasi-neutral flows, (II) Near-wake flow which refers to the wake in the vicinity of the body, (III) Far-wake flow which refers to the wake far downstream from the body.

In the present chapter, the flows in zone I are discussed. To best illustrate the characteristic distributions of particles and field in the flow. A model having the simplest two-dimensional configuration is used [18]. 


\subsection{SheAth}

Consider a collision-free mesothermal plasma stream, which is fully ionized, impinging on an electrically conducting plate at a small angle $\theta$ (Figure 14). It is assumed that the plate has a surface potential $\phi_{\mathrm{s}}$ which is negative relative to the ambient plasma. The incident electrons are absorbed; ions neutralized and re-emitted as neutrals. The plate is large or two-dimensional, therefore the edge effect is nil and the field potential can be treated as a one-dimensional problem $\phi=\phi(x)$. A further simplification is made by assuming that the value of the negative surface potential is high enough that only the electrons at the rare high energy tail of its distribution can reach the plate and be lost. Under such conditions, the electrons maintain quasi-equilibrium and have the Maxwell-Boltzmann distribution.

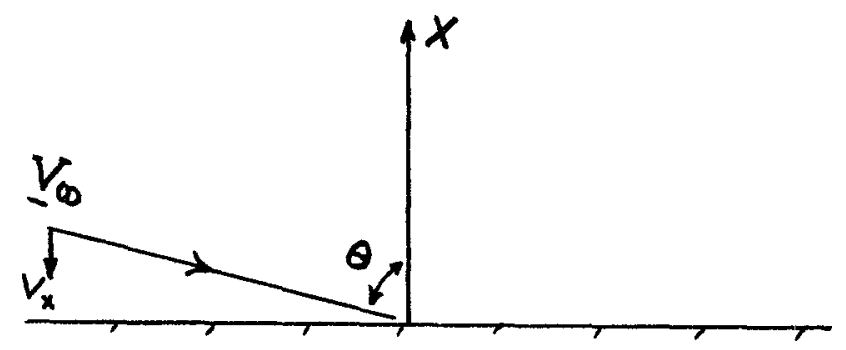

Fig. 14. Plasma sheath at an inclined plane with $\phi_{\mathrm{s}}<0, S_{x}=V_{x} / c_{\mathrm{i}}$.

$$
n_{\mathrm{e}}(\mathbf{r})=n_{\infty} \exp \left[e \phi(\mathbf{r}) / K T_{\mathrm{e}}\right]
$$

where $n_{\infty}$ denotes the electron density in the ambient plasma; it should be equal to the local ion density because the ambient plasma is neutral, i.e., $n_{\mathrm{i} \infty}=n_{\mathrm{e} \infty}=n_{\infty}$.

The self-consistent system of equations which govern the motion of the ions can be formulated [18] using the Schroedinger equation* and the Poisson equation.

$$
\begin{aligned}
& \partial^{2} \psi / \partial x^{2}+\partial^{2} \psi / \partial y^{2}+2 m_{\mathrm{i}} / \hbar^{2}[E-e \phi(x)] \psi=0 \\
& \mathrm{~d}^{2} \phi / \mathrm{d} x^{2}=-4 \pi e\left(n_{\mathrm{i}}-n_{\mathrm{e}}\right)=-4 \pi e n_{\infty}\left[|\psi|^{2}-\exp \left(e \phi / K T_{\mathrm{e}}\right)\right]
\end{aligned}
$$

with the boundary conditions:

$$
x=\infty, \quad \phi=0, \quad \mathrm{~d} \phi / \mathrm{d} x=0
$$

which says that the shield potential decays asymptotically to zero at the ambient plasma; also at

$$
x=0, \phi=\phi_{\mathrm{s}} .
$$

* An alternative method is to use the collision-free Boltzmann equation. 
In Equation (101), $E$ represents the energy of an ion in the incident stream relative to the reference frame fixed to the plate. The stream is approximately monoenergetic $(E)$ since $V_{\infty} \gg c_{\mathbf{i}}$. The discussion on the use of the Schroedinger equation for collisionfree plasma interaction has been given in Section 2.4, and will not be repeated here.

Let the wave function be represented as

$$
\psi(x, y)=\psi_{1}(x) \psi_{2}(y)
$$

as a trial for separation of variables in Equation (101). After the substitution for $\psi(104)$, Equation (101) is resolved into component equations:

$$
\begin{aligned}
& \mathrm{d}^{2} \psi_{1} / \mathrm{d} x^{2}+\left(2 m_{\mathrm{i}} / \hbar^{2}\right)[E-e \phi(x)] \psi_{1}=c_{1}^{2} \psi_{1} \\
& \mathrm{~d}^{2} \psi_{2} / \mathrm{d} x^{2}=c_{1}^{2} \psi_{2},
\end{aligned}
$$

where $c_{1}^{2}$ is a separation constant. Under WKBJ approximation [18] the solutions to Equations (105) and (106) can be readily obtained which, after the introduction of the upstream condition (103a), becomes

$$
|\psi|^{2}=\left(1-e \phi / E \cos ^{2} \theta\right)^{-1 / 2}
$$

It is interesting to note that the ion density function (107) agrees with that obtained by Hays [29] who used the collision-free Boltzmann equation, instead of Schroedinger equation for the ion distribution. It is also of interest to compare the present result with that of Ginzburg [34] who studied a special case $(\theta=0)$ of the problem using hydrodynamic equation suitable for continuum flows. Ginzburg's finding via continuum approach checks with the above result (107) when $\theta$ is set to zero and the pressureterm contribution in Ginzburg's result is neglected to comply with the present hyperthermal assumption.

Equation (102), after rearrangement and the substitution of $|\psi|^{2}(107)$, can be readily integrated [18]

$$
\begin{gathered}
x=\int_{\phi_{\mathrm{s}}}^{\phi}\left(8 \pi n_{\infty}\right)^{-1 / 2}\left\{2 E \cos ^{2} \theta\left(\sqrt{1-e \phi / E \cos ^{2} \theta}-1\right)-\right. \\
\left.-K T_{\mathrm{e}}\left[1-\exp \left(e \phi / K T_{\mathrm{e}}\right)\right]\right\}^{-1 / 2} \mathrm{~d} \phi .
\end{gathered}
$$

The sheath potential $\phi(x)$ (108), for a two-dimensional inclined plate facing a monoenergetic plasma stream, is given in an implicit form which has been plotted (Figure 15). It is observed from Figure 15 that the sheath characteristics, represented by the potential $\phi(x)$, the particle densities $n_{\mathrm{i}}(x)$ [Equation (107)], $n_{\mathrm{e}}(x)$ [Equation $(100)$ ], etc., all exhibit rapid variations nearest to the surface, in fact more than $95 \%$ of the drop in their values occurred in a minute distance from the surface which can 


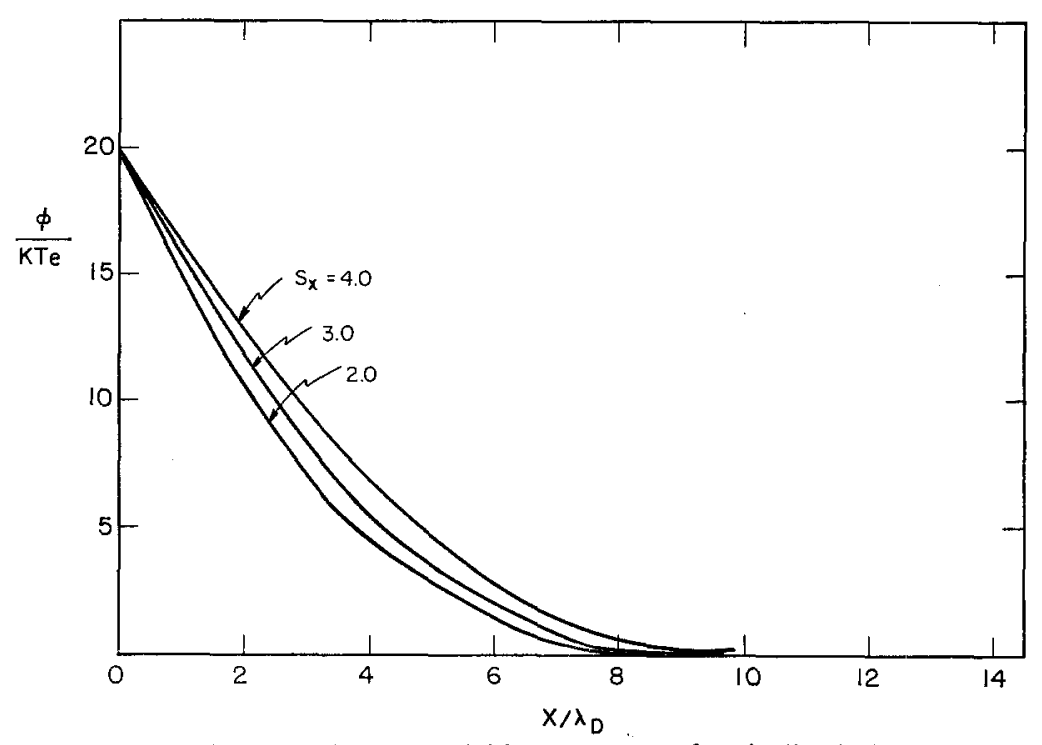

Fig. 15. Field potential in the sheath of an inclined plane.

be approximately defined as the thickness of the plasma sheath. This kind of boundary layer behavior [35] is inherent in the governing Equation (102) for the sheath potential $\phi$. It can be shown by non-dimensionalizing Equation (102) which then has a coefficient equal to $\left(\lambda_{\mathrm{D}} / L\right)^{2}$ in front of the Laplacian term (see Section. 2.2). Recall the ratio of the Debye shielding length $\left(\lambda_{\mathrm{D}}\right)$ to the characteristic dimension of the field $(L)$ is very small as assumed in the problem. This unique feature of the sheath has made it possible for the use of singular perturbation analysis in the stationary probe study [26].

The strong tendency for the variation of sheath characteristics to become onedimensional as shown in the papers of Langmuir [36] and others [29, 37] enhances the usefulness of the inclined plane solution. For instance, the sheath on a moving sphere can be determined by applying the inclined plate solution (107) to the local area of the frontal surface which has been suitably divided according to the local inclination relative to the free stream velocity $\left(V_{\infty}\right)$. The approach would be quite analogous to the Newtonian theory of sphere drag at hypersonic speeds [38] or the use of oblique shock relation to calculate the hypersonic sphere drag [39].

\subsection{Quast-Neutral flows}

It appears from the discussion in Section 5.1 that, beyond the sheath, the densities of ions and electrons are very nearly equal and the electric field is relatively weak, though still significant [21]. Generally speaking when a charged body moving in a tenuous ionosphere the charged particles in the ambient plasma are deflected, from their equilibrium distribution, both dynamically and electrostatically in the vicinity of the moving body. Although the charged particles tend to so arrange themselves in the disturbed zone as to shield the electric field carried by the moving body from 
penetrating into the ambient plasma. This shielding is not perfect. The flow region which is permeated by a weak, yet significant, field extension but with nearly equal ions and electrons densities $\left(n_{\mathrm{i}} \approx n_{\mathrm{e}}\right)$ everywhere is called a quasi-neutral flow. The quasi-neutral condition, $n_{\mathrm{i}} \approx n_{\mathrm{e}}$ essentially de-couple the field dependence on space charge as can be seen from Equation (102). The field however does provide the sole influence on the motion of the collision-free particles. In view of this, it is expected that a collision-free ionic flow in an attractive field is analogous to a corresponding neutral particle isothermal continuum flow with the field effect playing an equivalent role of the pressure gradient effect in the continuum flow as noted by Lam and Greenblatt [40]. In their innovation continuity and momentum equations for a continuum flow were used for a collisionless flow without any given justification. We shall establish this formal analogy using the wave mechanical formulation (see Section 2.4) to validate the continuum-like equations for representing the collisionless quasi-neutral flows.

It is assumed that the body has a surface potential which is negative relative to the ambient plasma and whose value is large enough that the loss of electrons to the surface is negligible. The incident stream is collision-free. It is also assumed that the body moves mesothermally hence the electron mass flow is discounted.

Under the present assumptions, only the ionic flow is considered. Consider the ionic flow which can be described by Schroedinger equation (see Section 2.4)

$$
i \hbar \frac{\partial \psi}{\partial t}=-\frac{\hbar^{2}}{2 m_{\mathbf{i}}} \nabla^{2} \psi+e \phi \psi,
$$

where the wave function $\psi$ depends on the coordinates (r) and of the time $(t)$. Similarly the potential $\phi$ may depend on both $\mathbf{r}$ and $t$. It is recalled that Schroedinger's original idea was to interpret $|\psi|^{2}$ as representing a true density, i.e., to have the particle smeared over space with mass density $\varrho=m_{\mathrm{i}}|\psi|^{2}$ and electric charge density $\varrho_{\mathrm{e}}=e|\psi|^{2}$. Later he reluctantly gave up his original idea and accepted Born's probability interpretation. Schroedinger's original idea can be renewed to interpret $|\psi|^{2}$ thus the classical picture of the system described by the wave function $\psi(\mathbf{r}, t)$ corresponds to a medium with density $\varrho(\mathbf{r}, t)$ spread out over space and moving under the influence of a field potential $\phi(\mathbf{r}, t)$, the 'elastic stresses' occurring inside the medium. Following this line of argument, Schroedinger already obtained the current [41, 42]

$$
n_{\mathrm{i}} \mathbf{v}=-\frac{i \hbar}{2 m_{\mathrm{i}}}\left(\psi^{*} \nabla \psi-\psi \nabla \psi^{*}\right)
$$

Equation (110), together with $n_{\mathbf{i}}=n_{\infty}|\psi|^{2}$ and Equation (109) satisfy the following relation

$$
\partial n_{\mathrm{i}} / \partial t+\operatorname{div}\left(n_{\mathrm{i}} \mathbf{v}\right)=0
$$

which is essentially the continuity equation in hydrodynamics [35] when it is multiplied by the ionic mass $m_{\mathrm{i}}$. 
The rate of change of the velocity ( $v$ ) of a fluid element as it moves about in space (r) can be written

$$
\frac{\mathrm{d} \mathbf{v}}{\mathrm{d} t}=\frac{\partial \mathbf{v}}{\partial t^{!}}+(\mathbf{v} \cdot \nabla) \mathbf{v}
$$

where $\partial v / \partial t$ denotes the change in $\mathbf{v}$ during $\mathrm{d} t$ at a point fixed in space while $(\mathbf{v} \cdot \nabla) \mathbf{v}$ gives the instantaneous difference between the velocities at two points $\mathrm{dr}$ apart, where $\mathrm{d} \mathbf{r}$ is the distance travelled by the given fluid element during $\mathrm{d} t$.

Differentiating relation (110) with respect to time and expressing the time derivatives with the use of Equation (109), we combine Equations (111) and (112) to obtain

$$
m_{\mathrm{i}} n_{\mathrm{i}} \frac{\mathrm{d} \mathbf{v}}{\mathrm{d} t}=-n_{\mathrm{i}} \nabla\left(e \phi-\frac{\hbar^{2}}{2 m_{\mathrm{i}}} \frac{\nabla^{2} \sqrt{n_{\mathrm{i}}}}{\sqrt{n_{\mathrm{i}}}}\right) .
$$

The second term in the parentheses of Equation (113) which appears only when the ion density is non-uniform plays the role of an inner potential as the result of 'deformation' which is of the order $\left(\lambda_{\mathrm{B}} / \lambda_{\mathrm{D}}\right)^{2} \cdot\left(m_{\mathrm{i}} c^{2} / 2\right)$, where $\lambda_{\mathrm{B}}$ is de Broglie wavelength of an ion with velocity $c$, and is negligible. We have now

$$
\varrho \frac{\mathrm{d} \mathbf{v}}{\mathrm{d} t}=-n_{\mathrm{i}} \nabla(e \phi)
$$

where the terms $-n_{\mathrm{i}} \nabla(e \phi)$, due to a force potential, plays an analogous role of a pressure gradient in an inviscid-like continuum flow in the absence of external force.

It is noted that Equations (111) and (114) provide a complete description of motions of a fluid which is incompressible or isothermal compressible.* In other words, if we impose an initial condition upon $\varrho$ and $\mathbf{v}: \varrho(\mathbf{r}, 0)$ and $v(\mathbf{r}, 0)$, the distributions $\varrho(\mathbf{r}, t)$ and $\mathbf{v}(\mathbf{r}, t)$ at a later time $(t)$ can be determined unique from Equations (111) and (113).

The above discussion, which starts from the motion of collision-free ions, leads to a description which is continuum-like. Thus we complete the proof of the formal analogy between a collisionless, quasi-neutral mesothermal flow and a corresponding isothermal compressible flow of continuous medium. A Kelvin-like theorem on conservation of circulation for the plasma can be established, accordingly, as in hydrodynamics [35]. The present discussion leads readily to the interesting hydrodynamic approximation, first suggested by Gurevich [17], for a plasma with $T_{\mathrm{i}}=T_{\mathrm{e}}=T$ by substituting $2 T$ for $T$ in the hydrodynamic equations for ion flow. It is valid because ions are under the influence of the sum of the partial pressures of ions and electrons, $n K\left(T_{\mathrm{i}}+T_{\mathrm{e}}\right)$ or $n K(2 T)$.

* A collisionless quasi-neutral flow can be considered isothermal because the fast-moving electrons help to equalize the 'temperature'. 


\subsection{SOLUTIONS OF QUASI-NEUTRAL FLOWS}

The quasi-neutral condition $n_{\mathrm{i}}=n_{\mathrm{e}}$ and the equilibrium electron distribution $n_{\mathrm{e}}=n_{\infty} \exp \left(e \phi / K T_{\mathrm{c}}\right)$ leads to a simple expression for the field potential

$$
\phi=\frac{K T_{\mathrm{e}}}{e} \ln \left(\frac{n_{\mathrm{i}}}{n_{\infty}}\right) .
$$

Thus in a quasi-neutral plasma, the body-plasma interaction problem reduces to the integration of the collision-free Boltzmann equation (or the Schroedinger equation if wave mechanical approach is used) for the distribution of the ions under a preserved field potential (115).

In analyzing collision-free mesothermal flows over a wedge and a cone with $\lambda_{\mathrm{D}} \ll L[40]$ [43], Lam and Greenblatt have suggested a boundary condition which matches the quasi-neutral flow to the outer edge of the sheath.

From the use of Equation (115) for quasi-neutral plasma, Gurevich and Pitayevsky [44] transformed the collisionless Boltzmann equation in terms of similarity variables to treat similar plasma flows over sharp edged bodies with $L \gg \lambda_{\mathrm{D}}$. Let $c^{*}=c / c_{\mathrm{i}}$, $\tau=[(L-X) / Z]\left(V_{\infty} / c_{\mathrm{i}}\right)$ where $X$ measures normal to the body surface; $Z$, along the undisturbed plasma flow $\left(V_{\infty}\right)$. The transformed equation for ion distribution assumes the form

$$
\left(c^{*}-\tau\right) \frac{\partial f}{\partial \tau}-\frac{1}{2} \frac{\partial f}{\partial c^{*}} \frac{\mathrm{d}}{\mathrm{d} \tau}\left\{\ln \int_{-\infty}^{\infty} f \mathrm{~d} c^{*}\right\}=0 .
$$

The solution of Equation (116) $f=f\left(c^{*}, \tau\right)$ is expressible in terms of similarity variables $c^{*}$ and $\tau$. The ionic distribution $f\left(c^{*}, \tau\right)$ have been obtained by numerical integration of Equation (116) for various values of $\tau$ in order to observe the evolution of $f$ with $\tau$. Some general conclusions concerning collisionless mesothermal flows can be obtained [44]. It should, however, be kept in mind that Equation (116) is valid for quasi-neutral flows only.

\section{Ionospheric Flows at Mesothermal Speeds. II : Near-Wake Flows}

When a charged body of size $L$, e.g., a satellite, drills through an ionospheric medium, having a characteristic mean free path $l$, at a mesothermal speed $V_{\infty}$, it momentarily creates a void region the transverse size of which is of the order of the body size. The rate of particle-filling into the vacuum, which determines its degree of emptiness, depends on the components of particle velocities normal to the direction of body motion, namely the random thermal components. Therefore, other than the electrostatic effect, the important parameter determining the structure of the flow immediately behind a moving body is the speed ratio $V_{\infty} / c_{\mathfrak{i}}$. The study of this particular flow is herein called the near-wake problem. 
The character of the binary species which are greatly different in their thermal speeds adds new complications to the near-wake problem. The electrons from the surroundings endowed with high thermal velocity move in quickly into the void and win the race over the thermally slower moving ions but not by far because of the mutual electrostatic attraction between them. The electron-ion race is, of course, also influenced by the biased influence of the surface potential $\left(\phi_{\mathrm{s}}\right)$. The above mentioned special features tend to make the collision-free plasma flow in the near wake a difficult but fascinating problem. The significance of this problem in the ionospheric diagnostics via satellites cannot be overemphasized. Often it is by necessity that a diagnostic probe for ionosphere or a radio-antenna is inserted into the near wake which is marked by intricate distributions of field and particles that imply complications in measurements and interpretations.

A point of paramount importance concerning theoretical analysis of the near wake is the following. In the earlier works on satellite-ionosphere interactions, to avoid the mathematical difficulty of nonlinear coupling between the collisionless particleEquation (3) and the field Equation (4), it is often assumed that the ions move like neutrals to simplify the space charge term in the field equation. This pseudo-neutral assumption is approximately valid for ions moving in the quasi-neutral and less but still reasonably true in the sheath because the directed kinetic energy $\left(E \approx \frac{1}{2} m_{\mathrm{i}} V_{\infty}^{2}\right)$ is much larger than the electrostatic potential energy $(e \phi)$ due to the field effect. This assumption would have no valid basis in applying to the near-wake study. As it has been stated earlier, the particle velocity that significantly contributes to the filling process for the void in the near wake is the thermal component, in other words the thermal energy $(3 K T / 2)$ which is comparable, in most cases, to the electrostatic potential energy $(e \phi)$. The neglect of the electric field effect, the implication of the pseudo-neutral approximation, in comparison with $K T_{\mathrm{i}}$ is unjustifiable.

A self-consistent approach, which takes account of the non-linear coupling between the particle- and the field-equations deserves even more attention in the case of near wake.

\subsection{A NEAR-WAKE MODEL}

In order to gain physical insight into the interaction between the field potential and the plasma particles associated with a rapidly moving body, an idealized model is introduced. Consider a body of size $L$ with a constant surface potential $\phi_{\mathrm{s}}\left(\phi_{\mathrm{s}} \leqslant 0\right)$ which moves in a rarefied plasma of singly charged particles. It is assumed that the free stream plasma is in a bi-thermal equilibrium which has an electron temperature $T_{\mathrm{e}}$ not necessarily equal to the ion temperature $T_{\mathrm{i}}$. The body velocity $V_{\infty}$ is assumed mesothermal. These conditions comply with typical satellite motion in the upper ionosphere (see Section 1.1). It is assumed that upon collision with the body an electron will be absorbed; an ion, neutralized and emitted as a neutral (see Section 1.2). The present model will satisfy the characteristic condition: $l \gg \mathrm{L} \gg \lambda_{\mathrm{D}}$. Magnetic field effect is considered negligible (see Table I).

In the following analysis, normalized quantities are used; e.g., the linear displace- 
ment $\mathbf{r}$ is in units of $L$; field potential $\phi$, in $K T / e$; velocity, in $c_{\mathrm{i}}$; number density $n$, in $n_{\infty}$ (the ambient electron density).

\subsection{SELF-CONSISTENT FIELD APPROACH}

Under the present assumptions of $\phi<0$ and $V_{\infty} \ll c_{\mathrm{e}}$, the electron density can be approximated by the Maxwell-Boltzmann distributions (in normalized units) [45]

$$
n_{\mathrm{e}}=\exp (\beta \phi)
$$

where $\beta=T_{\mathrm{i}} / T_{\mathrm{e}}$. It is, however, stipulated that $\phi(\mathbf{r})$ is a monotonic function of $\mathbf{r}$ [46]. A non-monotonic variation must be divided into monotonously, varying segments within each of which Equation (117) is applied. It will be seen later that this procedure is followed in the iteration for $\phi$ (see Section 6.4).

In view of the condition $V_{\infty} \gg c_{\mathrm{i}}$, the ion distribution in the wake is expected to deviate significantly from its equilibrium state. The ion distribution $f(\mathbf{r}, \mathbf{c})$ is governed by the collision-free Boltzmann equation, together with the Poisson equation for field potential $\phi(\mathbf{r})$ as follows:

$$
\begin{aligned}
\mathbf{c} \cdot \frac{\partial f}{\partial \mathbf{r}}-\frac{1}{2} \frac{\partial \phi}{\partial \mathbf{r}} \cdot \frac{\partial f}{\partial \mathbf{c}} & =0 \\
\left(\frac{\lambda_{\mathbf{D}}}{R}\right)^{2} \nabla^{2} \phi & =n_{\mathrm{e}}-n_{\mathrm{i}},
\end{aligned}
$$

where $n_{\mathrm{i}}=\int f \mathrm{~d}^{3} c$. It is noted that Equations (118) and (119) provide a self-consistent system.

In view of the earlier discussion, the use of pseudo-neutral particle approximation to decouple the system is not expected to be valid for the wake study. The application of Jeans theorem, expounded in Section 3.2, will be made here to obtain a formal solution for ion density $n_{\mathrm{i}}(\mathbf{r})$ from Equation (118). It is then substituted, together with relation (117), into Equation (119). The poisson equation, thus obtained, is a partial, non-linear, integro-differential equation which is solved by iteration.

\subsection{ION DISTRIBUTIONS}

According to Jeans' theorem, the most general solution to Equation (118) is an arbitrary function of the integrals of the system of characteristics equations deduced from Equation (118) namely

$$
\frac{-\mathrm{d} \mathbf{r}}{\mathbf{c}}=\frac{\mathrm{d} \mathbf{c}}{\frac{1}{2} \frac{\partial \phi}{\partial \mathbf{r}}} .
$$

The construction of the integrals for Equation (120) becomes difficult when the field potential is arbitrary. In fact the only known property of $\phi(\mathbf{r})$ is that, in the case of 


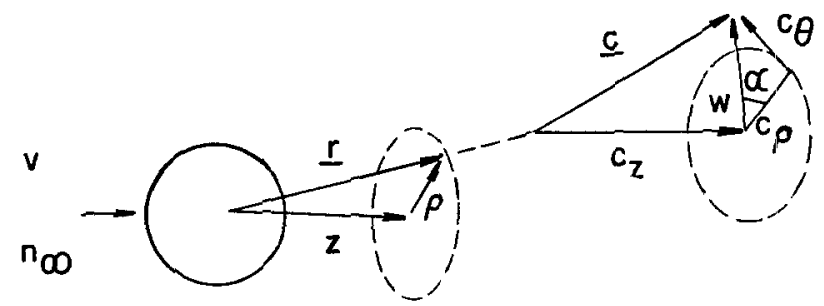

Fig. 16a. Phase space coordinates $\left(z, \varrho ; c_{\ell}, c_{\theta}, c_{z}\right)$ - an axi-symmetric wake (sphere).

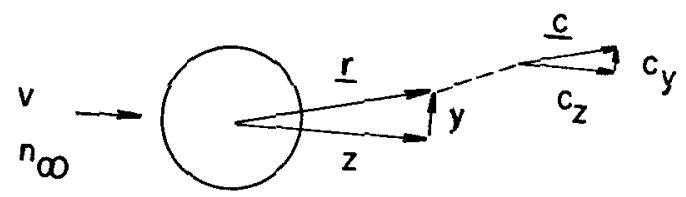

Fig. 16b. Phase space coordinates $\left(z, y ; c_{z}, c_{y}\right)-$ an $y$-symmetric wake (long cylinder).

a spherical body, it is axisymmetric with respect to the streamwise axis $(Z)$ passing through center of the sphere (see Figure 16a); in the case of a two-dimensional cylinder its plane of symmetry is given by the streamwise axis $(Z)$ and the axis of the cylinder $(x)$ (see Figure 16b). Resolve Equation (120) into its component forms: (I) Spherical body in phase space $\left(Z, \varrho ; c_{Z}, c_{\varrho}, c_{\theta}\right.$ ) (see Figure 16a); (II) Cylindrical body in phase space $\left(Z, y ; c_{Z}, c_{y}\right.$ ) (see Figure $16 \mathrm{~b}$ ). The Equations $(120)$ thus obtained can be integrated at once leading to the following adiabatic invariants [47]:

(i) Axisymmetric field $\phi(Z, \varrho)$ :

$$
\begin{aligned}
c_{\varrho}^{2}+c_{\theta}^{2}+c_{Z}^{2}+\phi & =E & & \text { Energy Conservation } \\
\varrho c_{\theta} & =L_{Z} & & \text { Angular Momentum Conservation }
\end{aligned}
$$

(ii) $Y$-symmetric field $\phi(Z, y)$ :

$$
c_{y}^{2}+c_{Z}^{2}+\phi=E \quad \text { Energy Conservation }
$$

where (i) is suitable for a sphere; (ii) for a two-dimensional cylinder along $x$-axis.

It is found that the general solutions to Equation (118) in terms of the above described invariants cannot provide complete descriptions of the ionic motions in the respective fields. One additional invariant in each of the above cases (i) and (ii) is needed. Under the conditions of hypersonic approximation $c_{Z}=V_{\infty}$ and sectionally monotonic $\phi(\mathbf{r})$, Jew [48] has been able to obtain a third integral (121c) for case (i) and a second integral (122b) for case (ii) which are valid for short ranges in $\mathbf{r}$ and are useful for numerical analyses. They are

$$
\begin{aligned}
I_{3}=\varrho-\frac{Z}{2 c_{Z}}\left(\frac{\sqrt{\varrho^{2}\left[E-\phi(Z, \varrho)-c_{Z}^{2}\right]-L_{Z}^{2}}}{\varrho}+\right. \\
+\frac{\sqrt{(\varrho+\eta)^{2}[E-\phi} \frac{\left.(Z-\eta, \varrho+\zeta)-c_{Z}^{2}\right]-L_{Z}^{2}}{\varrho+\zeta}}{}
\end{aligned}
$$


and

$$
I_{2}=y-\frac{Z}{2 c_{Z}}\left\{\sqrt{E-\phi(Z, y)-c_{Z}^{2}}+\sqrt{E-\phi(Z-\eta, y+\zeta)-c_{Z}^{2}},\right.
$$

where $\eta, \zeta \leqslant \lambda_{\mathrm{D}} / R$ denote the respective mesh sizes along $Z, \varrho$-axes with a sphere and $Z, y$-axes with a cylinder in numerical analyses.

The ionic distribution function $f(\mathbf{r}, \mathbf{c})$ which satisfies Equation (118) and the boundary conditions at the body and the free stream can be constructed with the help of Jeans' theorem [48], from which the ion densities are obtained.

(i) Axisymmetric field (sphere)

when $\varrho \leqslant 1$,

$$
\frac{n_{\mathrm{i}}}{n_{\infty}}=\frac{\exp (-\phi)}{\pi^{3 / 2}} \int_{-\infty}^{\beta_{\mathrm{s}}} \mathrm{d} c_{Z} \int_{0}^{2 \pi} \mathrm{d} \alpha \int_{0}^{\infty} \mathrm{d} w w \exp -\left[w^{2}+\left(c_{Z}-V_{\infty}\right)^{2}\right],
$$

where

$$
\beta_{\mathrm{s}}=\frac{Z w \cos \alpha}{2(\varrho+1)}\left[1+\sqrt{1+\frac{\phi(Z, \varrho)-\phi(Z-\eta, \varrho+\zeta)}{w^{2} \cos ^{2} \alpha}}\right]
$$

when $\varrho \geqslant 1$

$$
\begin{aligned}
& \frac{n_{\mathbf{i}}}{n_{\infty}}=\frac{\exp (-\phi)}{\pi^{3 / 2}}\left[\int_{-\infty}^{\infty} \mathrm{d} c_{Z} \int_{\sin ^{-1}(1 / \varrho)}^{2 \pi-\sin ^{-1}(1 / \varrho)} \mathrm{d} \alpha \int_{0}^{\infty} \mathrm{d} w+\int_{-\infty}^{\beta_{\mathrm{s}}} \mathrm{d} c_{Z} \int_{-\sin }^{\sin ^{-1}(1 / \varrho)} \mathrm{d} \alpha\right. \\
& \left.\times \int_{0}^{\infty} \mathrm{d} w+\int_{\gamma_{s}}^{\infty} \mathrm{d} c_{Z} \int_{\sin ^{-1} \operatorname{sil}_{(1 / \varrho)}^{-1}(1 / \varrho)} \mathrm{d} \alpha \int_{0}^{\infty} \mathrm{d} w w \exp -\left[w^{2}+\left(c_{Z}-V_{\infty}\right)^{2}\right]\right]
\end{aligned}
$$

where

$$
\gamma_{s}=\frac{Z w \cos \alpha}{2(\varrho-1)}\left[1+\sqrt{1+\frac{\phi(Z, \varrho)-\phi(Z-\eta, \varrho+\zeta)}{w^{2} \cos \alpha}} .\right.
$$

(ii) $Y$-symmetric field (cylinder)

when $y \leqslant 1$

$$
\begin{aligned}
\frac{n_{\mathrm{i}}}{n_{\infty}}=\frac{\exp (-\phi)}{2 \pi^{1 / 2}}\left[\int_{0}^{\infty} \exp \left(-c_{y}^{2}\right) \operatorname{erfc}\left(V_{\infty}+\gamma_{c}\right) \mathrm{d} c_{y}\right. \\
\left.+\int_{-\infty}^{0} \exp \left(-c_{y}^{2}\right) \operatorname{erfc}\left(V_{\infty}-\beta_{c}\right) \mathrm{d} c_{y}\right],
\end{aligned}
$$

where

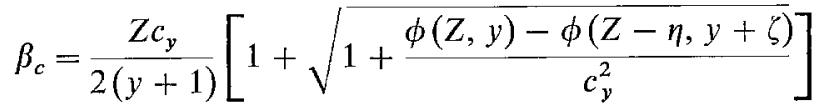


and

$$
\gamma_{c}=\frac{Z c_{y}}{2(y+1)}\left[1+\sqrt{1+\frac{\phi(Z, y)-\phi(Z-\eta, y+\zeta)}{c_{y}^{2}}}\right]
$$

and when $y \geqslant 1$

$$
\begin{gathered}
\frac{n_{\mathrm{i}}}{n_{\infty}}=\frac{\exp (-\phi)}{2 \pi^{1 / 2}}\left\{\int_{0}^{\infty} \exp \left(-c_{y}^{2}\right)\left[\operatorname{erfc}\left(V_{\infty}-\beta_{c}\right)+\operatorname{erfc}\left(\gamma_{c}-V_{\infty}\right)\right] \mathrm{d} c_{y}\right. \\
+\int_{-\infty}^{0} \exp \left(-c_{y}^{2}\right)\left[\operatorname{erfc}\left(V_{\infty}-\gamma_{c}\right)+\operatorname{erfc}\left(\beta_{c}-V_{\infty}\right)\right] \mathrm{d} c_{y} .
\end{gathered}
$$

\subsection{SELF-CONSISTENT FIELD}

The self-consistent solution to Equations (118) and (119) is obtained by successive iteration. The substitution of either set of ion density functionals (123) and (124) into Equation (119) leads to a non-linear integro-differential equation in $\phi(\mathbf{r})$ which does not yield to analytical attack. The boundary value problem can be prescribed as follows:

$$
\begin{aligned}
\left(\lambda_{\mathrm{D}} / R\right)^{2} \nabla^{2} \phi & =\exp \beta \phi-n_{\mathrm{i}}(\phi) / n_{\infty} \\
\phi(R) & =\phi_{\mathrm{s}} \\
\phi(\infty) & =0
\end{aligned}
$$

It is also postulated that the neutral plasma state $(\phi=0)$ exists on the transverse plane $(Z=0)$ external to the body. This amounts to the neglect of the sheath at the plane $Z=0$.

The problem just described is known as a Dirichlet boundary value problem. In the form stated above, it is not yet suitable for numerical analysis until a limited domain in physical space has been prescribed [45] due to finite memory of a computer, e.g., IBM (digital) 7090. To overcome this difficulty, the semi-infinite wake region is mapped into a semi-circular region coordinate inversion transformation [48, 49].

The iteration for $\phi(\mathbf{r})$ starts with a zeroth order solution $\phi^{(0)}$, obtained from $\nabla^{2} \phi^{(0)}=0$ (inner region) and $n_{\mathrm{i}}\left(\phi^{(0)}\right)=n_{\mathrm{e}}\left(\phi^{(0)}\right)$ (outer region) as suggested by Gurevich (see Section 2.3), and proceeds for higher order approximations [48, 49]. The sequence is repeated, each completed sequence is called a cycle. In each calculation, iteration cycles are carried out until a self-consistent state is attained. Normally this takes approximately 30 successive cycles. The mesh size used is smaller than a Debye length $\left(\lambda_{\mathrm{D}}\right)$. A specially devised iteration [48] makes the analysis possible.

The self consistent distributions of ion density $n_{\mathrm{i}}(\mathbf{r})$ and field potential $\phi(\mathbf{r})$ in the near wake of sphere and cylinder are shown separately in Figures 17-20. The electron density is related to $\phi(\mathbf{r})$ by Maxwell-Boltzmann relation (117). 


\subsection{Results AND Discussion}

The results of the self-consistent calculations for the particle and field distributions reveal some insight into the particle-field interactions in the near wake. We shall discuss their significances.

When a large conducting body $\left(L \gg \lambda_{\mathrm{D}}\right)$ with a negative surface potential $\left(\phi_{\mathrm{s}}<0\right)$ moves at a mesothermal speed in a tenuous ionosphere $(L \ll l)$, it is expected that both ions and electrons from the free stream will rush into the wake-cavity created by the moving body. The electrons, on the average, move faster; consequently the electronic and ionic components of the plasma always tend to separate. This gives rise to an uncompensated electronic charge which produces an internal electric field (the space charge potential) the latter retards the faster particles, namely the electrons, and accelerate the slower ones, i.e., the ions, thus hindering the charge separation. As a result, the inhomogeneous front moves and spreads with essentially a common mean speed, intermediate between those of the ions and the electrons; the internal electric field exerts in this case a strong influence on the particle motion as a whole in the near wake. The potential valleys in the wake of a sphere (see Figure 18) and of a cylinder (see Figure 20) are associated with the above mentioned fronts of electron-rich mixture. The locus of potential minima forms a conically shaped surface

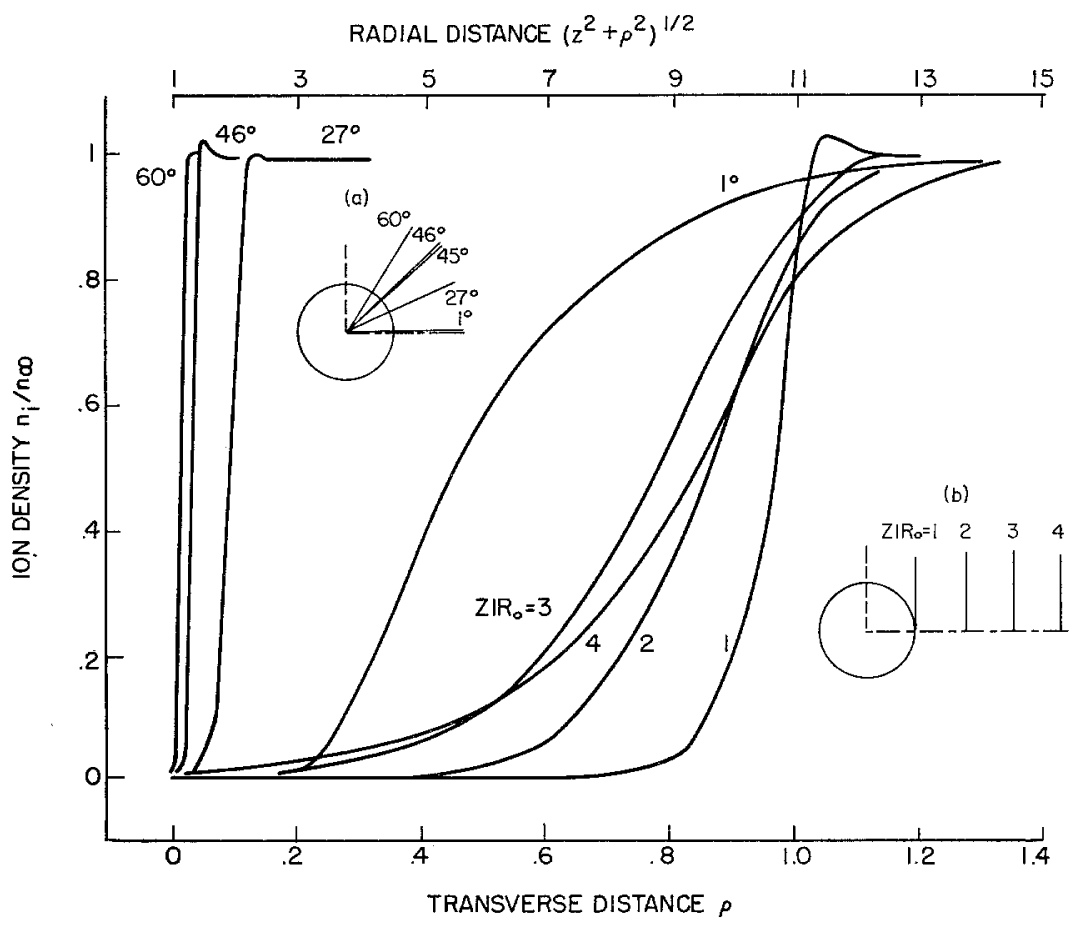

Fig. 17. Ion density in wake behind a sphere: $R / \lambda_{\mathrm{D}}=20, T_{\mathrm{i}} / T_{\mathrm{e}}=1, V_{\infty} / c_{\mathrm{i}}=8, e \phi_{\mathrm{S}} / K T_{\mathrm{i}}=-1$. 


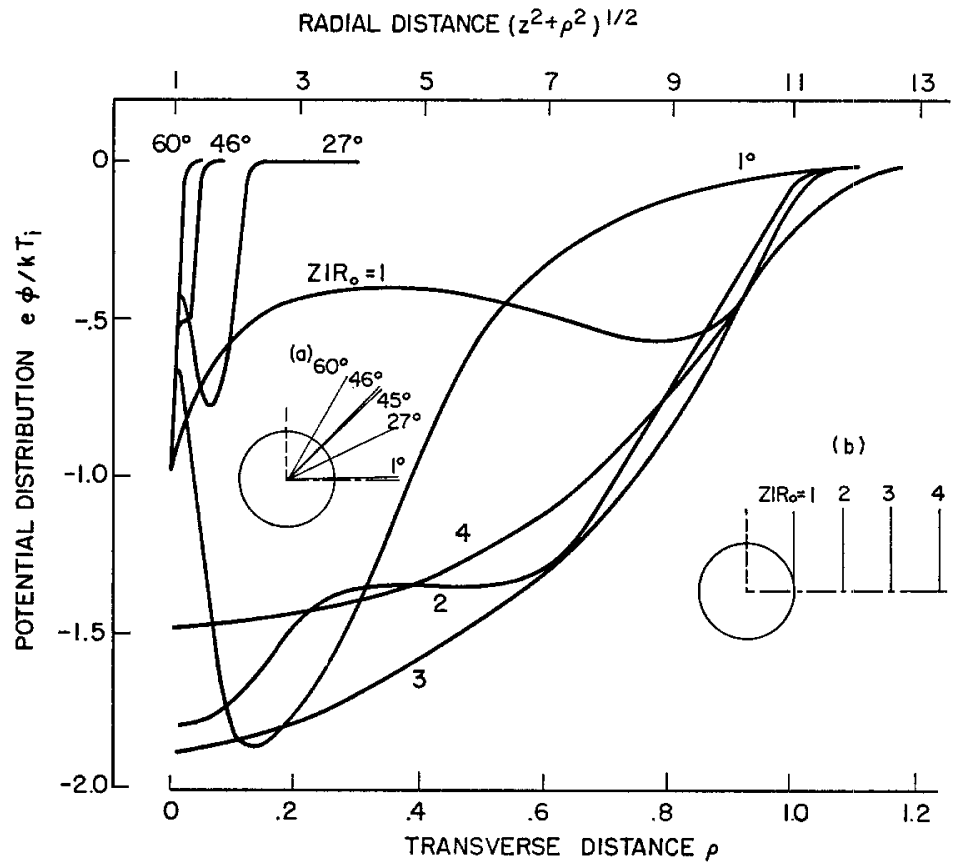

Fig. 18. Potential in wake behind a sphere:

$R / \lambda_{\mathrm{D}}=20, T_{\mathrm{i}} / T_{\mathrm{e}}=1, V_{\infty} / c_{\mathrm{i}}=8, e \phi_{\mathrm{S}} / K T_{\mathrm{i}}=-1$.

RADIAL DISTANCE $\left(z^{2}+y^{2}\right)^{1 / 2}$

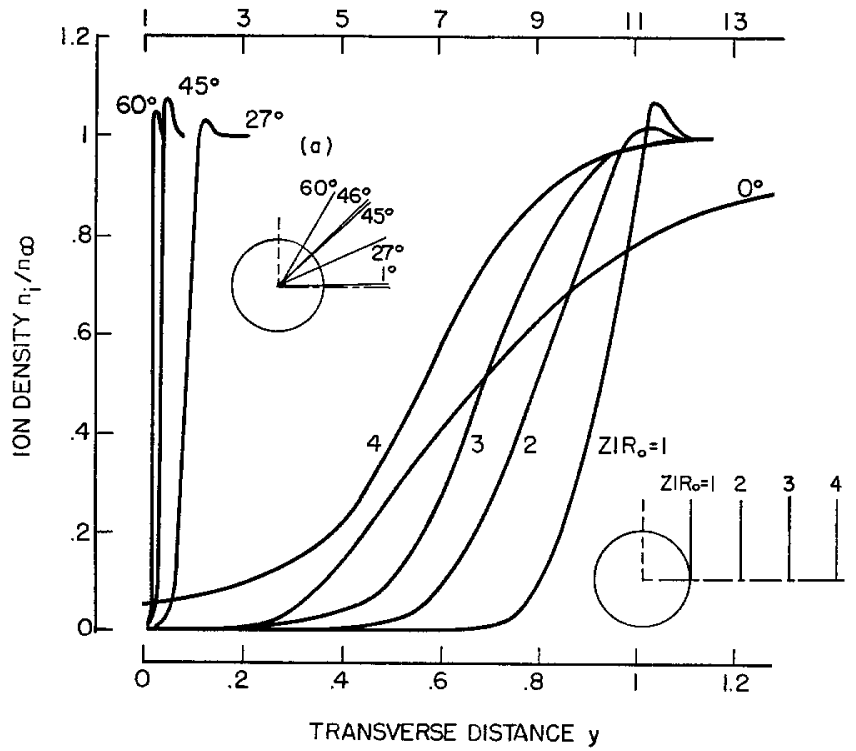

Fig. 19. Ion density in wake behind a transverse cylinder: $R / \lambda_{\mathrm{D}}=20, T_{\mathrm{i}} / T_{\mathrm{e}}=1, V_{\infty} / c_{\mathrm{i}}=8, e \phi_{\mathrm{s}} / K T_{\mathrm{i}}=-1$. 


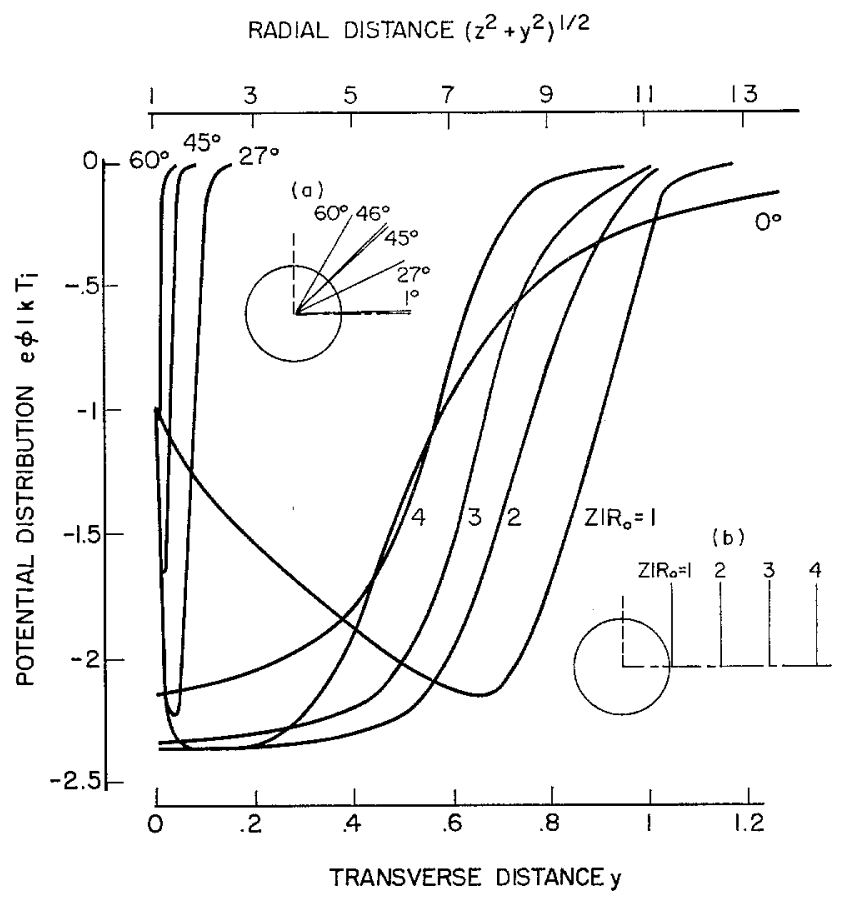

Fig. 20. Potential in wake behind a transverse cylinder: $R / \lambda_{\mathrm{D}}=20, T_{\mathrm{i}} / T_{\mathrm{e}}=1, V_{\infty} / c_{\mathrm{i}}=8, e \phi_{\mathrm{s}} / K T_{\mathrm{i}}=-1$.

for a spherical body; a double wedge for a cylindrical body. It is seen from the figures that the depth of the potential valley decreases as the body size $\left(R / \lambda_{\mathrm{D}}\right)$ decreases.

The presence of such potential valleys in the near wake of a satellite suggests the possible presence of trapped ions in the valley [50,51]. The specific modes of oscillations of the trapped ions and electrons will be discussed in Section 8.1.

\subsection{Photo-emission efFect}

From the discussion of Section 1.3, it is expected that at a relatively higher altitude, the photo-emission due to solar radiation tends to reduce the value of the negative surface potential. This would not affect the present self-consistent theory provided $\phi_{\mathrm{s}} \leqslant 0$. Another effect of the photo-emission stems from the emitted photoelectrons that enter the wake from the surface of the body. The electron-emission effect on the field potential of the near wake can be evaluated using a perturbation analysis [31] considering the present theory without emission effect as the zeroth order solution. The deviation of field and particle distributions due to perturbation emission effect can be obtained.

Assuming that $\phi(\mathbf{r})$ decreases, from $\phi_{\mathrm{s}}$ at the surface, to the $\phi_{\min }$ at the bottom of the potential, the photoelectrons, emitted from the surface, are in a repelling field. Thus the presence of these electrons has the effect of depressing the potential valley even deeper and building up a field which opposes the emission of electrons. 


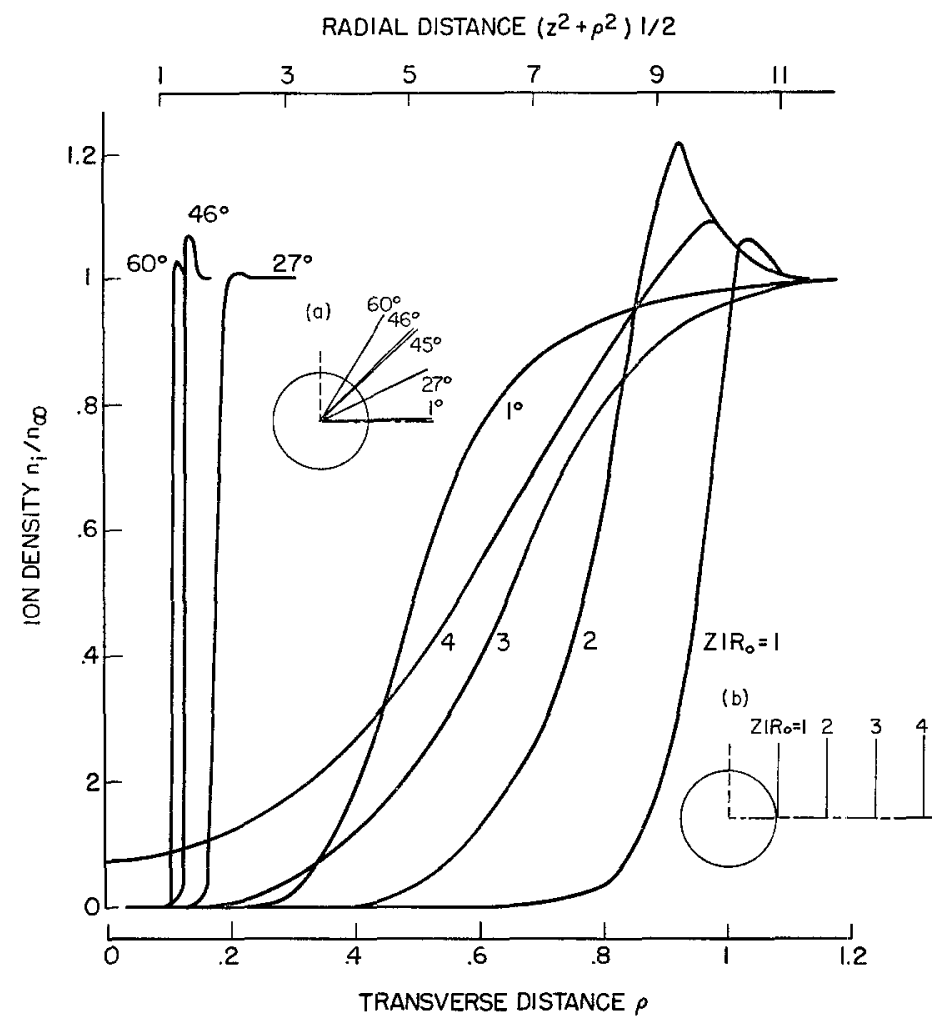

Fig. 21. Ion density in wake behind a sphere: $R / \lambda_{\mathrm{D}}=20, T_{\mathrm{i}} / T_{\mathrm{e}}=0.2, V_{\infty} / c_{\mathrm{i}}=8, e \phi_{\mathrm{s}} / K T_{\mathrm{i}}=-1$.

Consider the discussion in Section 1.1 on the bi-thermal nature $\left(T_{\mathrm{e}}>T_{\mathrm{i}}\right)$ of the upper ionosphere which is brought forth by the presence of high-energy photoelectrons. Assume that the ionosphere is in bi-thermal quasi-equilibrium with $T_{\mathrm{e}}=5 T_{\mathrm{i}}$, the surface potential $e \phi_{\mathrm{s}} / K T_{\mathrm{i}}=-1$, and the surface photo-emission is negligible, the results of self-consistent field calculation are shown in Figures 21 and 22.

\section{Ionospheric Flows at Mesothermal Speeds. III: Far-Wake Flows}

It is recalled that a dominant characteristic of the ionospheric gas dynamics herein discussed is its large Knudsen number i.e., $l / L \gg 1$ which, of course, implies rarity in binary collisions and hence leads to the collision-free state. On account of the rarity in collisions hence slower rate of dissipation in spite of collisionless damping, such as Landau damping, the disturbances, once generated, can persist for a long time or last a long distance behind the body. The structure of the disturbances undergoes much variation particularly in the near wake region which extends a distance of only a few body diameters. Thereafter the disturbed flow becomes almost quasi-neutral and persists for a relatively long period of time, during which collision-free damping 


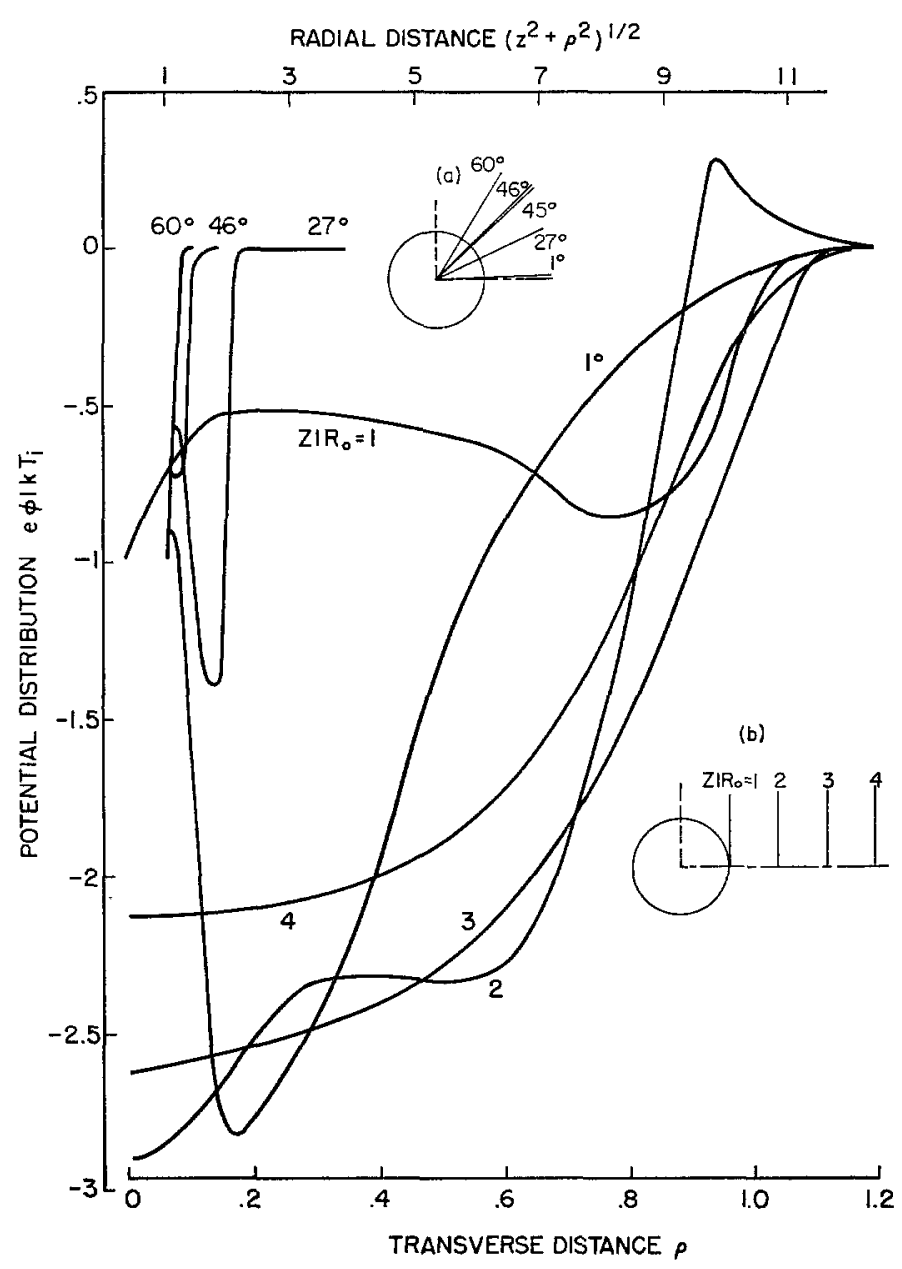

Fig. 22. Potential in wake behind a transverse cylinder: $R / \lambda_{\mathrm{D}}=20, T_{\mathrm{i}} / T_{\mathrm{e}}=0.2, V_{\infty} / c_{1}=8, e \phi_{\mathrm{S}} / K T_{\mathrm{i}}=-1$.

process acts to nullify the disturbances. The body-ionosphere interaction can be viewed in a different manner: The coulomb interaction of the charges carried by the moving body with the ambient plasma will excite oscillations in the wake of the body. The significant plasma disturbances will involve low frequency collective oscillations of the positive ions with the electrons adjusting themselves about the ions to form a Debye screening cloud. The long, yet shorter the mean free path $l_{\text {ei }}$, trail extending beyond the near wake is the subject of study in the far wake problem.

It is of interest to note that the unique feature of a great extension of plasma disturbances behind a moving body is not without technical significance as can be expected. For instance, it constitutes an excellent target for radiowave tracking of the moving body when the body itself does not provide large enough scattering cross 
section for the wave pulses because of long range. The plasma trail which has perturbation of the electron density that leads to the variation of the plasma dielectric constant which is responsible for the scattering effect. Although the dielectric constant of the trail is lower than a metallic body, it compensates in total scattering effect many fold over by its relatively large size.

The quasi-neutral, hence weak-field, nature of the far wake makes it possible to linearize the governing equations of the flow. The susceptibility of the far wake to linear analysis breeds much mathematical exercises which may not always lead to physical enlightening. On the other hand it is gratifying to explore an interesting physical phenomenon with a tool which is endowed with the luxury of mathematical elegancy.

\subsection{FAR-WAKE MODEL}

Consider a collisionless mesothermal flow far behind a moving body. Since the directed plasma velocity $\left(V_{\infty}\right)$ is negligibly small compared with the electron thermal velocity, the direct gas dynamic effect on the electron distribution is small. However the electron distribution is indirectly influenced through electrostatic field, e.g.,

$$
n_{\mathrm{e}}=n_{\infty} \exp (e \phi / K T)
$$

following the law of Maxwell-Boltzmann distribution.

It is of interest to study the nature of the disturbed plasma flow which has been influenced by the transport of disturbances orginating from far upstream. Only steady state properties are of concern here. The interesting, but quite difficult, problem of transcient phenomena is thereby avoided. Self-consistent field approach will be used to treat the collisionless flow of singly-charged ions which have interacted with a body; an energy of interaction $(\Omega)$ between them is introduced [52].

Let the coordinate system be fixed to the body. The collisionless Boltzmann equation for ionic distribution has the form

$$
c \cdot \frac{\partial f}{\partial \mathbf{r}}-\left(\frac{e}{m_{\mathbf{i}}} \frac{\partial \phi}{\partial \mathbf{r}}+\frac{1}{m_{\mathbf{i}}} \frac{\partial \Omega}{\partial \mathbf{r}}\right) \cdot \frac{\partial f}{\partial \mathbf{c}}=0 .
$$

Let $\mathbf{u}=\mathbf{c}-V_{\infty}$ which is substituted into Equation (126) that leads to

$$
\left(\mathbf{u}-V_{\infty}\right) \cdot \frac{\partial f}{\partial \mathbf{r}}-\left(\frac{e}{m_{\mathrm{i}}} \frac{\partial \phi}{\partial \mathbf{r}}+\frac{1}{m_{\mathrm{i}}} \frac{\partial \Omega}{\partial \mathbf{r}}\right) \cdot \frac{\partial f}{\partial \mathbf{u}}=0
$$

The field potential $\phi(\mathbf{r})$ in Equation (127) is governed by

$$
\nabla^{2} \phi=-4 \pi e\left[\int f \mathrm{~d}^{3} u-n_{\infty} \exp (e \phi / K T)\right]
$$




\subsection{Perturbation analysis}

It is expected that, at far downstream from the body, the ionic distribution must be close to the displaced Maxwellian distribution of the free stream

Assume

$$
f_{\infty}=n_{\infty}\left(\frac{m_{\mathrm{i}}}{2 \pi K T}\right)^{3 / 2} \exp -\frac{m_{\mathrm{i}} u^{2}}{2 K T}
$$

$$
f=f_{\infty}+f^{\prime},
$$

where the perturbation $f^{\prime}$ is small compared with $f_{\infty}$. The substitution of expression (130) into Equation (127) leads to

$$
\left(\mathbf{u}-V_{\infty}\right) \cdot \frac{\partial f^{\prime}}{\partial \mathbf{r}}-\left(\frac{e}{m_{\mathrm{i}}} \frac{\partial \phi}{\partial \mathbf{r}}+\frac{1}{m_{\mathrm{i}}} \frac{\partial \Omega}{\partial \mathbf{r}}\right) \cdot \frac{\partial f^{\prime}}{\partial \mathbf{u}}-\left(\frac{e}{m_{\mathrm{i}}} \frac{\partial \phi}{\partial \mathbf{r}}+\frac{1}{m_{\mathrm{i}}} \frac{\partial \Omega}{\partial \mathbf{r}}\right) \cdot \frac{\partial f_{\infty}}{\partial \mathbf{u}}=0 .
$$

Fourier transform of Equation (131) leads to

where

$$
\begin{aligned}
i \mathbf{\kappa} \cdot\left(\mathbf{u}-V_{\infty}\right) f_{\kappa}^{\prime} & -\left(e \phi_{\kappa}+\Omega_{\kappa}\right) \frac{\mathbf{\kappa}}{m_{\mathbf{i}}} \cdot \frac{\partial f_{\infty}}{\partial \mathbf{u}} \\
& +\frac{1}{(2 \pi)^{3} m_{\mathrm{i}}} \int\left[-i\left(e \phi_{\kappa^{\prime}}+\Omega_{\kappa^{\prime}}\right) \mathbf{\kappa}^{\prime}\right] \cdot \frac{\partial f_{\kappa-\kappa^{\prime}}^{\prime}}{\partial \mathbf{u}} \mathrm{d}^{3} \kappa^{\prime}=0,
\end{aligned}
$$

$$
\left[\begin{array}{l}
f_{\kappa}^{\prime} \\
\phi_{\kappa} \\
\Omega_{\kappa}
\end{array}\right]=\int \exp (-i \mathbf{\kappa} \cdot \mathbf{r})\left[\begin{array}{l}
f^{\prime} \\
\phi \\
\Omega
\end{array}\right] \mathrm{d}^{3} u .
$$

The far wake, where $r \rightarrow \infty$, corresponds to the condition $\kappa \rightarrow 0$ in the wave number ( $\kappa)$ space. The setting of $\kappa \rightarrow 0$ in Equation (132) leads to [52]

where

$$
i\left(\mathbf{u}-V_{\infty}\right) \cdot f_{\kappa}^{\prime}-i \frac{e}{m_{\mathrm{i}}} \mathbf{\kappa} \cdot \frac{\partial f_{\infty}}{\partial \mathbf{u}} \phi_{\kappa}=I(\mathbf{u})
$$

$$
\begin{aligned}
I(\mathbf{u}) & =\frac{1}{(2 \pi)^{3} m_{\mathrm{i}}} \int i\left(e \phi_{\kappa^{\prime}}+\Omega_{\kappa^{\prime}}\right) \boldsymbol{\kappa}^{\prime} \cdot \frac{\partial f_{\kappa-\kappa^{\prime}}}{\partial \mathbf{u}} \mathrm{d} \kappa^{\prime} \\
& =\frac{1}{m_{\mathrm{i}}} \int \frac{\partial f^{\prime}}{\partial \mathbf{u}} \cdot \frac{\partial}{\partial \mathbf{r}}(\Omega+e \phi) \mathbf{d}^{3} r .
\end{aligned}
$$

The application of similar Fourier transform and asymptotic analysis to Equation (128) yields

$$
\phi_{\kappa}=4 \pi e\left(\lambda_{\mathrm{D}}\right)^{2} \int f_{\kappa}^{\prime} \mathrm{d}^{3} u,
$$

where $\lambda_{\mathrm{D}}=\left(K T / 4 \pi e^{2} n_{\infty}\right)^{1 / 2}$ is the Debye shielding length. 
Divide Equation (134) by $i \kappa \cdot\left(u-V_{\infty}\right)$ and integrate over $\mathrm{d}^{3} u$. The improper integral whose integrand involving the factor $1 / i \mathbf{k} \cdot\left(\mathbf{u}-V_{\infty}\right)$ must be treated with special care [54]. Replace $\mathbf{\kappa} \cdot V_{\infty}$ by $\mathbf{\kappa} \cdot V_{\infty}+i \delta$, where $\delta \rightarrow \mathrm{O}^{+}$. The following equation is obtained $[52,53]$

$$
\int f_{\kappa}^{\prime} \mathrm{d}^{3} u-\frac{e}{m_{\mathbf{i}}} \phi_{\kappa} \int \frac{\mathbf{\kappa} \cdot \frac{\partial f_{\infty}}{\partial \mathbf{u}} \mathrm{d}^{3} u}{\mathbf{\kappa} \cdot\left(\mathbf{u}-V_{\infty}\right)-i \delta}=\frac{1}{i} \int \frac{I(\mathbf{u}) \mathrm{d}^{3} u}{\boldsymbol{\kappa} \cdot\left(\mathbf{u}-V_{\infty}\right)-i \delta} .
$$

Eliminating $\phi_{k}$ from Equations (134) and (136), it is found

$$
n_{\kappa}=\frac{\frac{1}{i \kappa} \int \frac{I(\mathbf{u}) \mathrm{d}^{3} u}{\mathbf{n} \cdot\left(\mathbf{u}-V_{\infty}\right)-i \delta}}{1+\left(\frac{m_{\mathrm{i}}}{2 \pi K T}\right)^{3 / 2} \int \frac{\mathbf{n} \cdot \mathbf{u}}{\mathbf{n} \cdot\left(\mathbf{u}-V_{\infty}\right)-i \delta} e^{-\frac{m_{\mathrm{i}} u^{2}}{2 K T}} \mathrm{~d}^{3} u},
$$

where $\mathbf{n} \equiv \mathbf{\kappa} /|\mathbf{k}|$

The Hilbert transform of Gaussian is defined

$$
Z(a)=\frac{1}{\pi i} \int_{-\infty}^{\infty} \frac{e^{-u^{2}}}{u-a-i \delta} \mathrm{d} u=e^{-a^{2}}\left(1+\frac{2 i}{\sqrt{\pi}} \int_{0}^{a} e^{t^{2}} \mathrm{~d} t\right) .
$$

In terms of $Z$-function, Equation (138) becomes

$$
n_{\kappa}=\frac{\frac{1}{i \kappa} \int \frac{I(\mathbf{u}) \mathrm{d}^{3} u}{\mathbf{n} \cdot\left(\mathbf{u}-V_{\infty}\right)-i \delta}}{2+i a Z(a)},
$$

where $a=\left(\mathbf{n} \cdot V_{\infty}\right) / c_{\mathrm{i}}$ and the ionic thermal velocity $c_{\mathrm{i}}=\sqrt{2 K T / m_{\mathrm{i}}}$.

\subsection{SOURCE INTEGRaL $I(\mathbf{u})$}

Pitayevsky and Kresin [52] named $I(\mathbf{u})$ 'collision integral' because it fills the place of the binary collision term in the Boltzmann equation when Equation (134) is transformed back to the earth-fixed coordinates. Looking from the standpoint of a far wake, $I(\mathbf{u}) \mathrm{d}^{3} u$ represents the number of particles per unit time which acquire, via scattering by the body, velocities in the range $(\mathbf{u}, \mathbf{u}+\mathrm{d} \mathbf{u})$, hence $I(\mathbf{u})$ is, instead, called a source integral [1] in order not to confuse with Boltzmann's collision integral. The introduction of $I(\mathbf{u})$ [52] gives an important conceptual advantage in the discussion of far wake. It also makes possible the convenient comparisons of the influences of bodies on the wake. Several forms of $I(\mathbf{u})$, each of which describes a special case of scattering, are available [52]. Only one of them will be discussed here for illustration.

In the case of a mesothermally moving body with a characteristic dimension $(L)$ large compared with Debye shielding length $\left(\lambda_{D}\right)$, the influence of the electric field in the sheath of the body upon the free stream ionic scattering would be small. Consider 
a sphere of radius $R_{0}$ and assume that ions incident on the spherical body are completely neutralized. $I(\mathbf{u})$ then denotes simply the number of the ions incident on the surface of the sphere in a unit time and is approximately [53]

$$
I(\mathbf{u}) \approx \pi R_{0}^{2} f_{\infty}(\mathbf{u})
$$

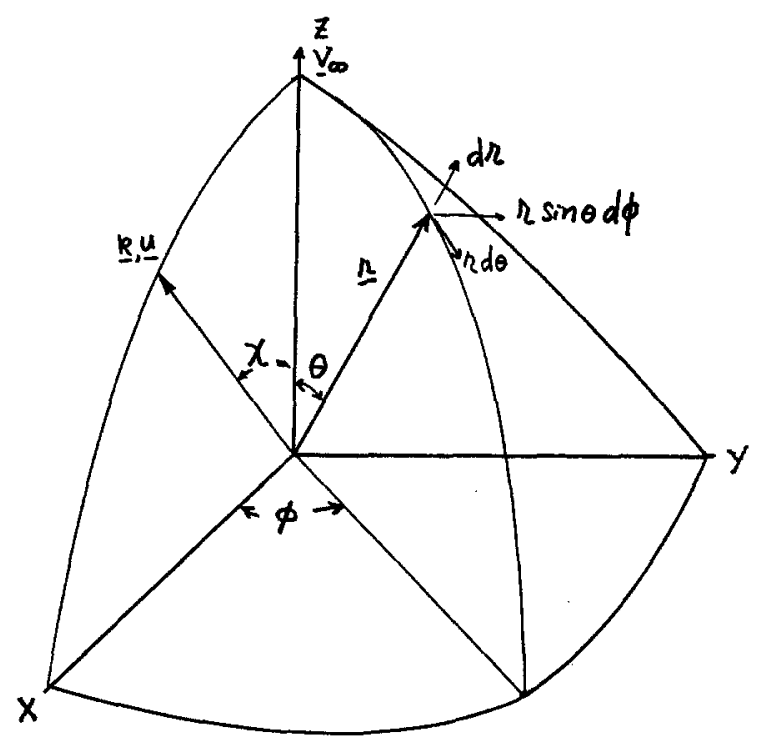

Fig. 23. Orientation of wave number space (к).

The Fourier-transformed ionic density in the far wake as given by Equation (138) can be simplified in the case of a large moving body $\left(L \gg \lambda_{\mathrm{D}}\right)$. Consider $V_{\infty}$ pointing in the direction of polar axis of a spherical coordinate (Figure 23); $\chi$ is the aximuth angle between the polar axis $\left(V_{\infty}\right)$ and the velocity $\mathbf{u}$ which is assumed parallel to к. Equation (138) becomes

$$
n_{\kappa}=\frac{\frac{1}{i \kappa} \int \frac{I(\mathbf{u}) \mathrm{d}^{3} u}{u-V_{\infty} \cos \chi-i \delta}}{2+\frac{V_{\infty} \cos \chi}{n_{\infty}}\left(\frac{m_{\mathbf{i}}}{2 \pi K T}\right)^{3 / 2} \int \frac{\exp \left(-m_{\mathrm{i}} u^{2} / 2 K T\right) \mathrm{d}^{3} u}{u-V_{\infty} \cos \chi-i \delta}} .
$$

The substitution of Equation (141) into Equation (142) gives, using function (139),

$$
n_{\kappa}=-\pi R_{0}^{2} S_{\mathrm{i}}^{2} \frac{n_{\infty}}{\kappa} \frac{Z\left(S_{\mathrm{i}} \cos \chi\right)}{2+i S_{\mathrm{i}} \cos \chi Z\left(S_{\mathrm{i}} \cos \chi\right)},
$$

where the speed ratio $S_{\mathrm{i}}=V_{\infty} / c_{\mathrm{i}}$. 
Let

$$
\Phi\left(S_{\mathrm{i}} \cos \chi\right)=\frac{Z\left(S_{\mathrm{i}} \cos \chi\right)}{2+i S_{\mathrm{i}} \cos \chi Z\left(S_{\mathrm{i}} \cos \chi\right)}
$$

Equation (143) can be written

$$
n_{\kappa}=-\pi R_{0}^{2} S_{\mathrm{i}}^{2} \frac{n_{\infty}}{\kappa} \Phi\left(S_{\mathrm{i}} \cos \chi\right)
$$

The study of the far wake structure would have been completed with $n_{\kappa}$ given by Equation (145), had the motivation been simply to investigate the scattering of an electromagnetic wave by the wake. This stems from the fact that, according to perturbation theories [52], the effective scattering cross-section depends among other factors on the Fourier-transformed electron-density perturbation which is approximately equal to that of the ion density in a far wake.

\subsection{ION DISTRIBUTION IN THE FAR WAKE}

The ion density in the far wake is of interest itself. To obtain an ion density from its counterpart in Fourier-transform $\left(n_{\kappa}\right)$, an inverse Fourier-transform must be performed. Again consider a large sphere, where $R_{0} \gg \lambda_{\mathrm{D}}$, at mesothermal speed, in the far wake

$$
n(\mathbf{r})=\frac{-\pi R_{0}^{2}}{(2 \pi)^{3}} S_{\mathrm{i}}^{2} n_{\infty} \int \Phi\left(S_{\mathrm{i}} \cos \chi\right) \exp (i \mathbf{\kappa} \cdot \mathbf{r}) \kappa^{-1} \mathrm{~d}^{3} \kappa
$$

Referring to Figure 23

$$
\begin{aligned}
& \mathrm{d}^{3} \mathbf{\kappa}=\sin \chi \mathrm{d} \chi \mathrm{d} \phi \kappa^{2} \mathrm{~d} \kappa \\
& \mathbf{\kappa} \cdot \mathbf{r}=\kappa r(\cos \theta \cos \chi+\sin \theta \sin \chi \cos \phi)
\end{aligned}
$$

which are substituted into integral (146).

$$
\begin{aligned}
n(r, \theta) & =\frac{-\pi R_{0}^{2}}{(2 \pi)^{2}} S_{\mathrm{i}}^{2} n_{\infty} \int_{0}^{\pi} \Phi\left(S_{\mathrm{i}} \cos \chi\right) \sin \chi \mathrm{d} \chi \int_{0}^{\infty} \kappa \mathrm{d} \kappa \int_{0}^{2 \pi} \exp \\
& \times\{i \kappa r(\cos \theta \cos \chi+\sin \theta \sin \chi \cos \varphi)\} \mathrm{d} \varphi .
\end{aligned}
$$

Introducing the relation

$$
\int_{0}^{2 \pi} e^{\mathrm{i} \alpha \cos \varphi} \mathrm{d} \varphi=2 \pi J_{0}(\alpha)
$$


to integral (147) which becomes

$$
\begin{array}{r}
n(r, \theta)=\frac{-\pi R_{0}^{2}}{(2 \pi)^{2}} S_{\mathrm{i}}^{2} n_{\infty} \int_{0}^{\pi} \Phi\left(S_{\mathrm{i}} \cos \chi\right) \sin \chi \mathrm{d} \chi \int_{0}^{\infty} \exp (i \kappa r \cos \theta \cos \chi) \\
\times J_{0}(\kappa r \cos \theta \cos \chi) \kappa \mathrm{d} \kappa .
\end{array}
$$

In the integration over $\mathrm{d} \kappa$, the following substitution* in the exponent of the integrand is made:

$$
\cos \theta \cos \chi \rightarrow \cos \theta \cos \chi+i \delta \quad\left(\delta \rightarrow \mathrm{O}^{+}\right)
$$

where $\delta$ has an infinitesimal positive value [54], the integration over $\mathrm{d} \kappa$ can now be performed as follows:

$$
\int_{0}^{\infty} e^{-\beta_{1} \kappa} J_{0}(\gamma \kappa) \kappa \mathrm{d} \kappa=\frac{\beta_{1}}{\left(\beta_{1}^{2}+\gamma^{2}\right)^{3 / 2}}, \text { for } R_{\mathrm{e}} \beta_{1}>0
$$

when integral (149) is inserted in the integral (148)

$$
\begin{aligned}
n(r, \theta) & =\frac{-\pi R_{0}^{2}}{(2 \pi)^{2} r^{2}} S_{\mathrm{i}}^{2} n_{\infty} \int_{0}^{\pi} \Phi\left(S_{\mathrm{i}} \cos \chi\right) \frac{\cos \theta \cos \chi \sin \chi \mathrm{d} \chi}{\left(\cos ^{2} \chi-\sin ^{2} \theta+2 \mathrm{i} \delta \cos \theta \cos \chi\right)^{3 / 2}} \\
& =-\frac{\pi R_{0}^{2} S_{\mathrm{i}}^{2} n_{\infty}}{(2 \pi r)^{2}} F_{\mathrm{L}}(\theta)
\end{aligned}
$$

where

$$
F_{\mathrm{L}}(\theta)=\int_{0}^{\pi} \Phi\left(S_{\mathrm{i}} \cos \chi\right) \frac{\cos \theta \cos \chi \sin \chi \mathrm{d} \chi}{\left(\cos ^{2} \chi-\sin ^{2} \theta+2 i \delta \cos \theta \cos \chi\right)^{3 / 2}}
$$

In the evaluation of $F_{L}(\theta)$, the following considerations are made. Since $\Phi\left(S_{\mathrm{i}} \cos \chi\right)$ is a function of analytic continuation in the entire domain of $\chi$, the singularities exist in the denominator of the integrand. Following Landau's concept of integration contour, the sign of the imaginary part of the denominator determines the integration path around the singularities. In what follows we shall consider only the region to the rear of the moving body i.e., $\pi / 2<\theta<\frac{3}{2} \pi$ hence $\cos \theta<0$ and the sign of the imaginary part in the parenthesis of the denominator is negative. Consequently the integration contour of interest take a path along the real axis in the positive imaginary plane [67].

* I am grateful to Mr. R. J. Hung for his help in evaluating the inverse Fourier transform. 
Let $\cos \theta=t$, the integral (151) becomes, after letting $\delta \rightarrow \mathrm{O}^{+}$,

$$
F_{\mathrm{L}}(\theta)=\cos \theta \int_{-1}^{1} \frac{\Phi\left(S_{\mathrm{i}} t\right) t \mathrm{~d} t}{[(t-\sin \theta)(t+\sin \theta)]^{3 / 2}} .
$$

Further advance in the evaluation of integral (152) can be made by identifying its singularities at the points : $t=-\sin \theta$ and $t=\sin \theta$ and using Cauchy integral.

$$
\begin{aligned}
F_{\mathrm{L}}(\theta) & =\cos \theta\left\{R_{\mathrm{e}} \Phi^{\prime}\left(S_{\mathrm{i}} \sin \theta\right) \ln \sin \theta+\int_{\sin \theta}^{1} R_{\mathrm{e}} \Phi^{\prime \prime}\left(S_{\mathrm{i}} t\right) \ln \left(t+\sqrt{t^{2}-\sin ^{2} \theta}\right)\right. \\
& \times \mathrm{d} t+\left[\frac{\pi}{2} I_{\mathrm{m}} \Phi^{\prime}\left(S_{\mathrm{i}} \sin \theta-\int_{0}^{\sin \theta} I_{\mathrm{m}} \Phi^{\prime}\left(S_{\mathrm{i}} t\right) \sin ^{-1} \frac{t}{\sin \theta} \mathrm{d} t\right]\right\}
\end{aligned}
$$

where $\Phi^{\prime}(x) \equiv \mathrm{d} \Phi / \mathrm{d} x$ and $\Phi^{\prime \prime}(x)=\mathrm{d}^{2} \Phi / \mathrm{d} x^{2}$.

This essentially completes the evaluation of $n(r, \theta)$. It is noted that $n(\mathbf{r})$ varies with $1 / r^{2}$ when $r$ is large (see Equation (150)).

The applications of the far wake plasma study will be given in Section 8.2.

\section{Special Topics of Ionospheric Gas Dynamics}

There are numerous ionospheric gas dynamic problems which do not fit directly into the scope of studies in the previous sections. An encyclopedical coverage of these problems is not the intent of the present review. It might be, however, of interest to both ionospheric physicists and gas dynamicists, to note that some problems of keenest interest in ionosphere can be treated effectively in the light of ionospheric gas dynamics, e.g., the problems of radio meteors, comet dynamics, etc. The choice of topics herein discussed is obviously dictated by personal interest. It is, however, intended that each topic should have a unique feature.

\subsection{Particle trapping in The NEAR WAKE}

Recent measurements of the ionosphere using satellites have produced some interesting but unexpected results: the electrostatic probe studies of electron density and temperature of the ionosphere in the wake of the Ariel I satellite showed the possible presence of low-frequency plasma oscillations [55]. Plasma resonances found with the Alouette topside sounder satellite occur at multiples of certain characteristic frequencies of high frequency plasma oscillations [56, 57, 58]. A hypothesis has been advanced [59] that longitudinally oscillating ions in the potential well behind a mesothermally moving satellite are responsible for the above-mentioned low-frequency oscillations measured in the wake of Ariel I. It also suggests that the high frequency electronic plasma oscillations shown in the ionograms of Alouette satellite are associated with the low-frequency oscillations of the trapped ions. 
This hypothesis has been made in view of the following observations: (1) The consistency and persistence of the characteristic oscillations observed in the Alouette ionogram suggests that the source and site of these plasma oscillations might be of limited extent and of long duration, possibly associated with the motion of the satellite. (2) A self-consistent field theory of near wake behind a mesothermally moving satellite [45] reveals a negative potential well, the depth of which amounts to three volts with a negative surface potential less than one volt. This is about the level of surface potential for the satellites in question (see Figure 18). On the basis of the above-mentioned observations of experimental and theoretical results, it was concluded that ion particles must have been trapped in the potential well of the near wake executing longitudinal oscillations in the potential well.

To furnish a quantitative and therefore more convincing evidence for his hypothesis, Liu [50] used a one-dimensional model, what he called a diode analogue, with the free stream identified as its cathode; satellite surface, its anode, to derive a dispersion for the standing waves of the ionic oscillations in the potential well. This derivation uses an approximation similar to the Langmuir-Tonks derivation for a homogeneous plasma that uses a simplified two-fluid hydrodynamic approach. Although Liu's dispersion relation shows that the theoretically predicted ionic oscillations does agree in the order of magnitude with the oscillations measured on Ariel I, it leaves much unanswered questions, e.g., the validity of hydrodynamic approach, the microscopic instability of the plasma in the potential well, etc. A kinetic theory of non-homogeneous plasma oscillations, such as the plasma in a potential well, and microscopic instability problem is being prepared by Liu and Hung [61]. It is trusted that this new theory will be able to answer some of these pertinent questions pertaining to particles trapped in a well.

\subsection{ElECTRO-GAS DYNAMIC THEORY OF METEOR}

A meteor is a stony or metallic body that has fallen to the earth from outer space. A metallic meteor moving in the ionosphere must accrete charges from the incident ions and electrons of the ambient plasma much like an orbiting satellite (see Section 1.3). A charged meteor behaves electro-gas dynamically as a satellite except that most of them move at a higher speed before they disintegrate and evaporate by entering into the denser atmosphere.

It would be of interest to study the far wake of a metallic meteor particularly one in a meteor shower where a cloud of meteoric particles is found. It is noted that when a meteor (B) is located in the wake of a meteor (A), the plasma in the wake of B could develop parametric resonance due to the presence of $A$. It is recalled that parametric resonance condition can be met for a plasma in an external periodic electric field. Here the external periodic field for B is provided by the disturbance field of $A$. This resonance-like plasma phenomenon has been shown to occur with two bodies in tandem arrangement aligned with a mesothermal stream [60]. The presence of this resonance with the multiple scattering of plasma stream by a meteor shower could account for the seemingly anomalous scattering cross section of an electro-magnetic wave. 
In the study of the above-mentioned resonance phemonema [60], Liu and Hung use two charged bodies in a mesothermal stream. The ionospheric medium is assumed bi-thermal, namely the electron temperature $\left(T_{\mathrm{e}}\right)$ not necessarily equal to the ion temperature. Each particle species is in thermal quasi-equilibrium. Their analysis for the double scattering is essentially a continuation of the simple scattering analysis in Section 7.4. The body (B), which is situated in the far wake of body (A), receives a disturbed incident stream from $\mathrm{A}$. Another way of viewing the body-scattering problem is to consider the steady state plasma wave excitation. With mesothermal speed and absence of magnetic field effect, the only mode of significance is the ionic longitudinal oscillation. Hence the scattering process can be treated mathematically in the Fourier-transformed space as a linear system with incident stream as input and longitudinal ionic oscillations in the far wake as output of the system; the transfer function which links the output to the input is provided by the equations of selfconsistent field approach, namely the collisionless Boltzmann equation and the Poisson equation. This system analysis can be extended to any steps of successive scatterings in a straightforward manner.

\subsection{INITIAL RADIUS OF AN IONIZED METEOR TRAIL}

When a meteor enters into the earth's atmosphere, the aerodynamic heating of the meteor surface is so intense that evaporation of meteoric atoms takes place. The atoms which evaporate from the meteor are dispersed in the surrounding atmosphere. Their kinetic energies are essentially determined by the velocity of the meteor, and for iron atoms will vary from about 100 to 1000 electron volts for velocities from 20 to $60 \mathrm{~km} / \mathrm{sec}$ [62]. The collisions occurs between the evaporated meteoric atoms and the atoms of the atmosphere, also between the atmospheric atoms incident on and reflected from the meteoric surface. The probabilities for excitation and ionization in these collisions determine the eventual electron line density of the meteor trail [63] which, in turn, determines the detectability of radio echo from the meteor in question [64]. One of the problems pertaining to the detectability of radio echo is the initial radius of ionized meteor trails [65].

The ions and electrons produced by the above-mentioned collisions in a meteor trail form a cloud of a quasi-neutral plasma whose concentration at the time of plasma formation is higher than the concentration of ionospheric plasma at those altitudes. It is often assumed that the ionization process is instantaneous and the meteor-generated plasma cloud dissipates by turbulent or ambipolar diffusion. Since the initial widening of the trail occurs mainly as a result of the first few mean free paths of the evaporated or reflected particles, the 'diffusion' process during the initial mean free time* becomes important. It is well-known that the diffusion equation in parabolic form does not represent the true physical process near the initial instant of time $(t=0)[66,67]$ because the classical diffusion equation results from an asymptotic approximation of the process when time $(t)$ is large compared with the mean free time [68].

* Equal to the mean free path length divided by the particle mean speed. 
To provide a more rigorous description of diffusion, the Boltzmann equation (with binary collision term included) should be used. This naturally involves with heavy mathematical computation. It is of interest to note that when the distribution function $f(\mathbf{r}, \mathbf{c}, t)$ is expanded in terms of spherical harmonics and substituted into the Boltzmann equation, and moments are made to take advantage of the orthogonality of harmonics, a system of continuum-like equations at various orders of spherical harmonics will be obtained [69]. At the level of first order approximation, a generalized diffusion equation is obtained in the form of telegraph equation, which has, in addition to the dispersion-term of classical diffusion equation, a wave-propagation term. This is very significant because at the initial instant $(t>0)$ prior to the first (mean) molecular collision, the imposed initial concentration discontinuity should be propagated wave-like at approximately thermal speed of the molecules.

This telegraph equation for diffusion can be easily derived following either Weinberg and Wigner [69] or Goldstein [68]. The latter uses random function method to treat stochastic processes [47]. The telegraph equation for concentration of diffusing particles $C(\mathbf{r}, t)$ can be written

$$
\nabla^{2} C=\frac{1}{D} \frac{\partial C}{\partial t}+\frac{1}{A^{2}} \frac{\partial^{2} C}{\partial t^{2}},
$$

where $A=v / \sqrt{3}$ with $v$ denoting the monoenergetic particle velocity; the extraneous factor $1 / \sqrt{3}$ is a consequence of the first order spherical harmonic approximation. A most recent work by Liu [72] who uses the equation of transfer of Maxwell [15] shows that $A^{2}=k T / m$ where $T$ is the effective kinetic temperature of the evaporated meteor atoms.

Notice that in Equation (154), the wave-term is usually negligibly small compared with the dispersion-term except near $t=0$ when the rate of change of concentration is extremely high in the time scale of a mean free time.

The Green's function of Equation (154) which describes the concentration at $\mathbf{r}$ caused by a unit point source at the origin $r=0$ [32]

$$
\begin{aligned}
C(r, t)=\frac{A}{r} & \exp \left(-\frac{1}{2} \frac{A^{2}}{D} t\right)\{\delta(A t-r) \\
& \left.+\frac{A r}{2 D \sqrt{r^{2}-A^{2} t^{2}}} J_{1}\left[\frac{1}{2} \frac{A}{D} \sqrt{r^{2}-A^{2} t^{2}}\right] H(A t-r)\right\},
\end{aligned}
$$

where the step function

$$
H(x)= \begin{cases}0 ; & x<0 \\ 1 ; & x>0\end{cases}
$$

and $J_{1}$ denotes the first-order Bessel function of the first kind [32]. 
For illustration of the physical process, the source solution to the one dimensional form of Equation (154) is used [32]

$$
C_{1}\left(x_{1} t\right)=2 \pi A \exp \left(-\frac{1 A^{2}}{2 D} t\right) J_{0}\left[\frac{A}{2 D} \sqrt{x^{2}-A^{2} t^{2}}\right] H(A t-x),
$$

where $J_{0}$ denotes the zeroth-order Bessel function of the first kind. It is observed from solution (156) that the concentration disturbance that gives the initial discontinuity is confined to within the signal zone, $x \leqslant A t$. The concentration at a point $x_{1}$ remains undisturbed until the instant when $x_{1}=A t_{1}$. At this time which is that required for a disturbance to travel from the origin $(x=0)$ to $x_{1}$, a concentration wave with intensity $\sim \exp \left(-A^{2} t_{1} / 2 D\right)$ passes through $x_{1}$. After this initial wave the slower dispersion process takes over. For comparison of concentration at a point, $x_{1}=D / 2 A$, can be made between the concentration $C_{1}(D / 2 A, t)$ described by the telegraph equation and by the classical diffusion equation (Figure 24) for the initial conditions:

$$
\partial C / \partial t \mid \begin{aligned}
& =0 \\
& t=0
\end{aligned}
$$

for all $x, C(x, 0)=0$ for $x>0$ and $C(x, 0)=1$ for $x<0$ which represent appropriately a meteor source of interest.

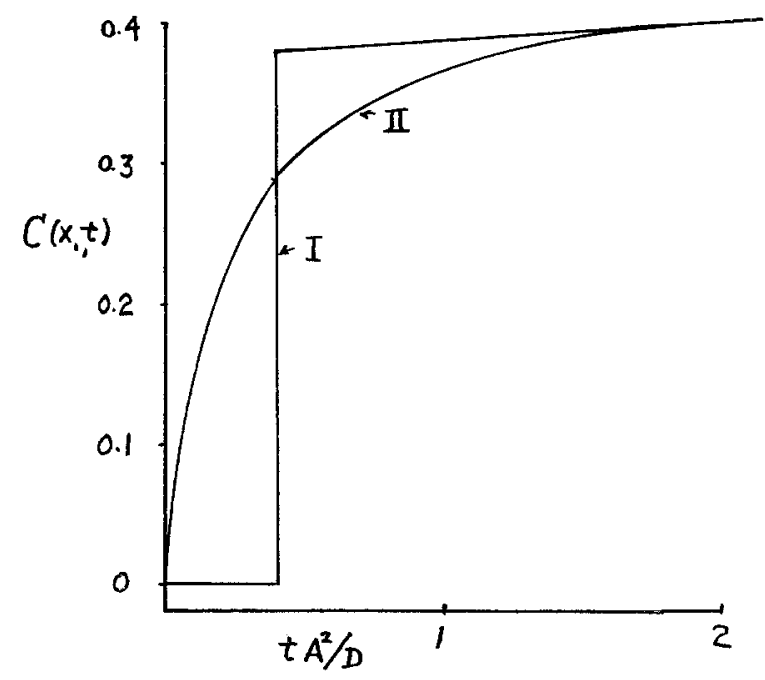

Fig. 24. Comparison of fixed-point concentration variations $C\left(x_{1}, t\right)$ - telegraph equation (I) vs. classical diffusion equation (II).

Notice that the time scale $D / A^{2}$ is on the order of a mean free time. The results shown in Figure 24 imply that the two solutions become undistinguishable after 1.5 mean free time. This is an extremely small time for gases at normal density. On the 
other hand at a high altitude where the meteor trail dispersion is of interest, it may become significant. The above discussion of dispersion is based on a coordinate fixed to meteor body, hence it is a stationary source. In actual application to a moving meteor, a transformation of the equation to the earth-fixed system must be made [70]. This would make the disparity between the two solutions in Figure 24 more pronounced. No matter how the initial radius of the ionized meteor trail is defined, the discrepancy in concentration profiles near $t=0$ should be of interest.

\section{Acknowledgment}

This work and our earlier results on ionospheric gas dynamic studies are made possible by the NASA Grant NsG-660 and NGR 23-005-094.

\section{References}

\section{A. CITED REFERENCES}

[1] Al'pert, Ya. L., Gurevich, A. V., and Pitayevsky, L. P.: 1963, Space Sci. Rev. 2, 680; also Soviet Phys. USPEKHI 6, 13.

[2] Al'pert, Ya. L.: 1965, Space Sci. Rev. 4, 375.

[3] Chopra, K. P.: 1961, Rev. Mod. Phys. 33, 153.

[4] Chen, F. F.: 1965, Plasma Diagnostic Techniques (ed. by R. H. Huddlestone and S. L. Leonard) Academic Press, New York, ch. 4.

[5] Kagan, Y. M. and Perel, V. I.: 1964, Soviet Phys. USPEKHI 6, 767.

[6] Brace, L. H., Spencer, N. W., and Dalgarno, A.: 1965, Planetary Space Sci. 13, 647.

[7] Schmerling, E. R.: 1966, Rev. Geophys. 4, 329.

[8] Gringauz, K. I., Bezrukikh, V. V., and Ozerov, V. D.: 1961, Artificial Earth Satellites (ed. by L. V. Kurnosova), Plenum Press, New York, vol. VI, p. 77 (Transl.).

[9] Helliwell, R. A.: 1965, Whistlers and Related Ionospheric Phenomena, Stanford University Press, Stanford.

[10] Massey, H. S. W., and Burhop, E. H. S.: 1952, Electronic and Ionic Impact Phenomena, Oxford University Press, London.

[11] McDaniel, E. W.: 1964, Collision Phenomena in Ionized Gases, John Wiley, New York.

[12] Kurt, P. G. and Moroz, V. I.: 1962, Planetary Space Sci. (Transl.) 9, 259.

[13] Brundin, C. L.: 1963, Am. Inst. Aeron. Astronaut. J. 1, 2529.

[14] Montgomery, D. C. and Tidman, D. A.: 1964, Plasma Kinetic Theory, McGraw-Hill, New York.

[15] Chapman, S. and Cowling, T. G.: 1939, The Mathematical Theory of Non-Uniform Gases, Cambridge University Press, New York.

[16] Liu, V. C., Pang, S. C., and Jew, H.: 1965, Phys. Fluids 8, 788.

[17] Gurevich, A. V.: 1962, Planetary Space Sci. (Transl.) 9, 321.

[18] Liu, V. C.: 1965, Nature 208, 883.

[19] Schiff, L. I.: 1955, Quantum Mechanics, McGraw-Hill, New York.

[20] Bernstein, I. B. and Rabinowitz, I. N.: 1959, Phys. Fluids 2, 112.

[21] Laframboise, J. G.: 1966, UTIAS Rept. No. 100 (University of Toronto).

[22] Watson, K. M.: 1956, Phys. Rev. 102, 12.

[23] Goldstein, H.: 1959, Classical Mechanics, Addison-Wesley, Reading, Mass.

[24] Sonin, A. A.: 1966, Am. Inst. Aeron. Astronaut. J. 4, 1588.

[25] Chen, F. F.: 1965, J. Nucl. Energy C7, 47.

[26] Lam, S. H.: 1965, Phys. Fluids 8, 73.

[27] De Leeuw, J. H.: 1963, Physico-Chemical Diagnostics of Plasma (ed. by T. P. Anderson, R. W. Springer, and R. C. Warder Jr.), Northwestern University Press, Evanston, Ill.

[28] Chen, F. F., Etievant, C., and Mosher, D.: 1968, Phys. Fluids 11, 811.

[29] Hays, P. B.: 1964, A Study of Some Problems in the Kinetic Theory of Rarefied Gases in an 
Externa ]Force Field, Ph.D. Thesis, University of Michigan. (Available from University Microfilm Co., Ann Arbor, Michigan).

[30] Chmielewski, G. E.: 1968, Electrostatic Probes in a Slowly Drifting Rarefied Plasma, Ph.D. Thesis, University of Michigan (Available from University Microfilm Co., Ann Arbor, Michigan).

[31] Van Dyke, M.: 1964, Perturbation Methods in Fluid Mechanics, Academic Press, New York.

[32] Morse, P. M. and Feshbach, H.: 1953, Methods of Theoretical Physics, vols. I and II, McGrawHill, New York.

[33] French, J. B.: 1965, UTIA Rept. No. 79 (University of Toronto).

[34] Ginzburg, M. A.: 1962, ARS J. (Suppl.) 32, 1794.

[35] Landau, L. D. and Lifshitz, E. M.: 1959, Fluid Mechanics, Addison-Wesley, Reading, Mass.

[36] Langmuir, I.: 1961, Collected Works of Irving Langmuir 5 (ed. by G. Suits) Macmillan, New York.

[37] Allen, J. E., Boyd, R. L. F., and Reynolds, P.: 1957, Proc. Phys. Soc. (London) B70, 297.

[38] Hayes, W. D. and Probstein, R. F.: 1959, Hypersonic Flow Theory, Academic Press, New York.

[39] Liu, V. C.: 1958, J. Appl. Phys. 29, 194.

[40] Lam, S. H. and Greenblatt, M.: 1968, Rarefied Gas Dynamics, vol. II, (ed. by J. H. deLeeuw) Academic Press, New York, p. 45.

[41] Madelung, E.: 1926, Z. Physik 40, 332.

[42] Schroedinger, E.: 1926, Ann. Physik. 79, 361, 489; 80, 437; 81, 109.

[43] Lam, S. H. and Greenblatt, M.: 1965, Am. Inst. Aeron. Astronaut. J. 3, 1850.

[44] Gurevich, A. V. and Pitayevsky, L. P.: 1965, Phys. Rev. Letters 15, 346.

[45] Liu, V. C. and Jew, H.: 1967, Rarefied Gas Dynamics, vol. II, (ed. by C. L. Brundin), Academic, Press, New York, p. 1703.

[46] Lam, S. H.: 1967, Rarefied Gas Dynamics, vol. II, (ed. by C. L. Brundin), Academic Press, New York, p. 1603.

[47] Chandrasekhar, S.: 1960, Principles of Stellar Dynamics, Dover, New York.

[48] Jew, H.: 1968, Numerical Studies of the Rarefied-Plasma Interactions at Mesothermal Speeds, Ph.D. Thesis, University of Michigan (Available from University Microfilm Co., Ann Arbor, Michigan).

[49] Liu, V. C. and Jew, H.: 1968, A.I.A.A. Preprint paper No. 68-169.

[50] Liu, V. C.: 1967, Nature 215, 127.

[51] Liu, V. C.: 1969, Space Res., vol. IX, North-Holland Publ. Co., Amsterdam.

[52] Pitayevsky, L. P. and Kresin, V. Z.: 1961, Soviet Phys. JETP 13, 185.

[53] Pitayevsky, L. P.: 1961, Geomagnetizm i Aeronomiya 1, 172.

[54] Jackson, J. D.: 1960, J. Nucl. Energy C1, 171.

[55] Samir, U. and Willmore, A. P.: 1965, Planetary Space Sci. 13, 285.

[56] Fejer, J. A. and Calvert, W.: 1964, J. Geophys. Res. 69, 5049.

[57] Crawford, F. W., Harp, R. S., and Mantei, T. D.: 1967, J. Geophys. Res. $72,57$.

[58] Dougherty, J. P. and Monaghan, J. J.: 1965, Proc. Roy. Soc. (London) 289, 214.

[59] Sturrock, P. A.: 1965, Phys. Fluids 8, 88.

[60] Liu, V. C. and Hung, R. J.: 1968, Planetary Space Sci. 16, 845.

[61] Liu, V. C. and Hung, R. J.: 1969, 'Oscillations of Electrostatically Trapped Particles' (to be submitted).

[62] Öpik, E. J.: 1958, Physics of Meteor Flight in the Atmosphere, Interscience Publishers, New York.

[63] Liu, V. C.: 1954, Rept. on the Study of Meteoric Ionization, Project M679 Problem 145, Radiation Lab. Internal Memo. (unpublished), University of Michigan.

[64] Kaiser, T. R.: 1953, Adv. in Phys. (Phil. Mag. Suppl.) 1, 495.

[65] Hawkins, G. S. and Hall, J. E.: 1960, Monthly Notices Roy. Astron. Soc. 121, 183.

[66] Jaffe, G.: 1942, Phys. Rev. 61, 643.

[67] Weymann, H. D.: 1967, Am. J. Phys. 35, 488.

[68] Goldstein, S.: 1951, Quart. J. Mech. Appl. Math. 4, 129.

[69] Weinberg, A. M. and Wigner, E. P.: 1958, The Physical Theory of Neutron Chain Reactors, University of Chicago Press, Chicago.

[70] Carslaw, H. S. and Jaeger, J. C.: 1959, Conduction of Heat in Solids, Oxford University Press, London.

[71] Allis, W. P. and Rose, D. J.: 1954, Phys. Rev. 93, 84.

[72] Liu, V. C.: 1969, 'Initial Radius of an Ionized Meteor Trail' (to be submitted). 
B. GENERAL REFERENCES

\section{Physical Properties of the Ionosphere}

1. Friedman, H.: 1962, Proc. Intern. Conf. Ionosphere, vol. III, (ed. by A. C. Stickland), Inst. Phys. London., p. 3.

2. Bowhill, S. A.: 1962, Proc. Intern. Conf. lonosphere, vol. III, (ed. by A. C. Stickland), Inst. Phys, London, p. 43.

3. Ferraro, V. C. A., Gliddon, J. E. C., and Kendall, P. C.: 1962, Proc. Intern. Conf. Ionosphere, vol. III, (ed. by A. C. Stickland), Inst. Phys. London, p. 71.

4. Hines, C. O.: 1962, Proc. Intern. Conf. Ionosphere, vol. III, (ed. by A. C. Stickland), Inst. Phys. London. p. 233.

5. Matsushita, S.: 1962, Proc. Intern. Conf. Ionosphere, vol. III, (ed. by A. C. Stickland), Inst. Phys. London, p. 120.

6. Willmore, A. P., Boyd, R. L. F., and Bowen, P. J.: 1962, Proc. Intern. Conf. Ionosphere, vol. III (ed. by A. C. Stickland), Inst. Phys. London, p. 517.

7. Sayers, J.: 1962, Proc. Intern. Conf. Ionosphere, vol. III, (ed. by A. C. Stickland), Inst. Phys. London, p. 523.

8. Chapman, J. H.: 1965, Electron Density Profles in Ionosphere and Exosphere (ed. by J. Frihagen), North-Holland Publ. Co., Amsterdam, p. 264.

9. Bauer, S. J.: 1965, Electron Density Profiles in Ionosphere and Exosphere (ed. by J. Frihagen), North-Holland Publ. Co., Amsterdam, p. 270.

10. Calvert, W.: 1965, Electron Density Profles in Ionosphere and Exosphere (ed. by J. Frihagen), North-Holland Publ. Co., Amsterdam, p. 280.

11. Thomas, J. O., Rycroft, M. J., Colin, L., and Chan, K. L.: 1965, Electron Density Profiles in Ionosphere and Exosphere (ed. by J. Frihagen), North-Holland Publ. Co., Amsterdam, pp. $299,322$.

12. Brace, L. H. and Reddy, B. M.: 1965, Electron Density Profiles in Ionosphere and Exosphere (ed. by J. Frihagen), North-Holland Publ. Co., Amsterdam, p. 397.

13. Doupnik, J. R. and Schmerling, E. R.: 1965, J. Atmospheric Terrest. Phys. $27,917$.

14. Spencer, N. W., Brace, L. H., Carigan, G. R., Taeusch, D. R., and Nieman, H.: 1965, J. Geophys. Res. 70, 2665.

15. Storey, L. R. O.: 1953, Phil. Trans. Roy. Soc. (London) A246, 113.

16. Ratcliffe, J. A., Schmerling, E. R., Setty, C. S. G. K., and Thomas, J. O.: 1956, Phil. Trans. Roy. Soc. (London) A248. 621.

17. King, J. W., Smith, P. A., Eccles, D., Fooks, G. F., and Helm, H. : 1964, Proc. Roy. Soc. (London) A281, 464.

18. Nagy, A. F., Brace, L. H., Carigan, G. R., and Kanal, M.: 1963, J. Geophys. Res. 68, 6401.

19. Gringauz, K. I.: 1958, Soviet Phys. Doklady, 3, pt. 2, 620.

20. Gringauz, K. I.: 1961, Space Res., vol. II, p. 583.

21. Hanson, W. B.: 1962, Space Res., vol. III, p. 282.

22. Hanson, W. B. and Johnson, F. S.: 1961, Mem. Soc. Roy. Sci. (Liège) 4, 390.

23. Hanson, W. B.: 1962, J. Geophys. Res. 67, 183.

24. Hinteregger, H. E. and Watanabe, K.: 1962, J. Geophys. Res. 67, 3373.

25. Dalgarno, A., McElroy, M. B., and Moffett, R. J.: 1963, Planetary Space Sci. $11,463$.

26. Evans, J. V. and Lowenthal, M.: 1964, Planetary Space Sci. 12, 915.

27. Bauer, S. J.: 1963, Nature 197, 36.

28. Bourdeau, R. E.: 1964, Space Res., vol. V, p. 77.

29. Bourdeau, R. E. and Donley, J. L.: 1964, Proc. Roy. Soc. (London) A281, 487.

30. Bourdeau, R. E., Whipple, E. C., Donley, J. L., and Bauer, S. J.: 1962, J. Geophys. Res. $67,467$.

31. Bowen, P. J., Boyd, R. L. F., Henderson, C. L. and Willmore, A. P.: 1964, Proc. Roy. Soc. (London) A281, 504.

32. Bowen, P. J., Boyd, R. L. F., Raitt, W. J., and Willmore, A. P.: 1964, Proc. Roy. Soc. (London) A281, 526.

33. Kurt, V. G.: 1964, Soviet Phys. USPEKHI 6, 701.

34. Lagow, H. E. and Horowitz, R.: 1958, Phys. Fluids 1, 478.

35. Boyd, R. L. F. and Laflin, S.: 1968, Proc. Roy. Soc. A307, 449.

36. Walker, J. C. G. and Spencer, N. W.: 1968, Science 162, 1437. 


\section{Microscopic Surface Accommodation}

1. Hagstrum, H. D.: 1954, Phys. Rev. 96, 325, 336.

2. Francis, G.: 1960, Ionization Phenomena in Gases, Academic Press, New York.

3. Von Engel, A.: 1965, Ionized Gases, Oxford University Press, London.

4. Fletcher, N. H. and Haydon, S. C.: 1964, Fundamental Surface Process in Discharge and Plasma Physics (ed. by S. C. Haydon), Department of Univ. Ext., University of New England, Armidale, Australia.

5. Wehner, G. K.: 1955, Advances in Electronics and Electron Physics (ed. by L. Marton), vol. VII, Academic Press, New York, p. 239.

6. Görlich, P.: 1959, Advances in Electronics and Electron Physics (ed. by L. Marton), vol. VII, Academic Press, New York, p. 11.

\section{Equilibrium Surface Potential}

1. Weissler, G. L.: 1956, Photoionization in Gases and Photoelectric Emission from Solids, Handbuch der Physik, vol. XXI, Springer Verlag, Berlin, p. 304.

2. Bordeau, R. E., Donley, J. L., Serbu, G. P., and Whipple, E. C.: 1961, J. Aeronaut. Sci. 8, 65.

3. Gringauz, K. I. and Zelikman, M. Kh.: 1957, USPEKHI Fiz. Nauk. 63, 108.

4. Hinteregger, H. E., Damon, K. R., and Hall, L. A.: 1959, J. Geophys. Res. 64, 961.

5. Rees, M. H. and Rense, W. A.: 1959, J. Geophys. Res. 64, 1251.

\section{Methods of Plasma Kinetics}

1. Bubkev, G. I. and Beliaev, S. T.: 1957, Plasma Physics and Problems of Controlled Thermonuclear Reactions, vol. II, (USSR), Pergamon Press, New York, p. 431.

2. Landau, L. D.: 1946, J. Phys. (USSR) 10, 25.

3. Spitzer, Jr., L.: 1956, Physics of Fully Ionized Gases Interscience, New York.

4. Rostoker, N. and Rosenbluth, M.: 1960, Phys. Fluids 3, 1.

5. Bernstein, I. B. and Trehan, S. K.: 1960, Nucl. Fusion 1, 3.

6. Van Kampen, N. G.: 1955, Physica 21, 949.

7. Case, K. M.: 1959, Ann. Phys. (N. Y.) 7, 349.

8. Dungey, J. W.: 1958, Cosmic Electrodynamics, Cambridge University Press, Cambridge.

9. Thompson, W. B.: 1962, An Introduction to Plasma Physics, Pergamon Press, New York.

\section{Wave-Mechanical Approach}

1. Bohm, D. and Vigier, J. P.: 1954, Phys. Rev. 96, 208.

2. Takabayasi, T.: 1952, Progr. Theoret. Phys. (Japan) 8, 143.

3. Takabayasi, T.: 1953, Progr. Theoret. Phys. (Japan) 9, 187.

4. Bohm, D.: 1953, Phys. Rev. 89, 1458.

5. Keller, J.: 1953, Phys. Rev. 89, 1040.

6. Bohm, D.: 1952, Phys. Rev. 85, 166, 180.

7. Babikov, V. V.: 1967, Soviet Phys. USPEKHI 92, 271.

8. Van Horn, H. M. and Salpeter, E. F.: 1967, Phys. Rev. 157, 751.

6. Electrostatic Probe

1. Langmuir, I.: Collected works of Irving Langmuir, vol. III (ed. by G. Suits), Macmillan, New York, p. 4.

2. Hall, L. S. and Freis, R. P.: 1965, Univ. Calif. Lawrence Rad. Lab. Rept. No. UCRL-12450.

3. Hall, L. S.: 1961, Univ. Calif. Lawrence Rad. Lab. Rept. No. UCRL-6535.

4. Hall, L. S.: 1964, Univ. Calif. Lawrence Rad. Lab. Rept. No. UCRL-7660-T.

5. Walker, E. H.: 1965, Interactions of Space Vehicles with an ionized Atmosphere (ed. by S. F. Singer), Pergamon Press, London.

6. Bettinger, R. T. and Walker, E. H.: 1965, Phys. Fluids 8, 748.

7. Medicus, G.: 1961, J. Appl. Phys. 32, 2512.

8. Medicus, G.: 1962, J. Appl. Phys. 33, 3094.

9. Chen, F. F.: 1962, Nuovo Cimento 26, 698.

10. Chen, F. F.: 1965, J. Nucl. Energy, C7, 47.

11. Self, S. A.: 1963, Phys. Fluids 6, 1762. 
12. Bohm, D.: 1949, The Characteristics of Electrical Discharges in Magnetic Fields (ed. by A. Guthrie and R. K. Wakerling), McGraw-Hill, New York.

13. Ecker, G. and McClure, J. J.: 1962, Z. Naturforsch. 17a, 705.

14. Harrison, E. R. and Thompson, W. B.: 1959, Proc. Phys. Soc. (London) 14, pt. 2, 145.

15. Auer, P. L.: 1961, Nuovo Cimento 22, 548.

16. Caruso, A. and Cavaliere, A.: 1962, Nuovo Cimento 26, 1389.

17. Allen, J. E. and Magistrelli, F.: 1960, Nuovo Cimento 18, 1138.

18. Dote, T., Takagama, K., and Ichimiya, T.: 1962, J. Phys. Soc. (Japan) 17, 174.

19. Allen, J. E. and Thonemann, P. C.: 1954, Proc. Phys. Soc. (London) B67, 768.

20. Waymouth, J. F.: 1964, Phys. Fluids 7, 1843.

21. Sonin, A. A., Graf, K. A., Laframboise, J. G., and De Leeuw, J. H.: 1965, 7th Int. Conf. on Phenomena in Ionized Gases.

22. Kanal, M.: 1964, J. Appl. Phys. 35, 1697.

\section{Plasma Sheath}

1. Wharton, C. B.: 1963, Physico-Chemical Diagnostics of Plasma (ed. by T. P. Anderson, R. W. Springer and R. C. Warder, Jr.), vol. III, Northwestern University Press, Evanston, Ill.

2. Loeb, L. B.: 1955, Basic Processes of Gaseous Electronics, University of Calif. Press, Berkeley, Calif.

3. Wasserstrom, E., Su, C. H., and Probstein, R. F.: 1965, Phys. Fluids 8, 56.

4. Hohl, F. and Wood, G. P.: 1963, Rarefied Gas Dynamics, vol. II, (ed. by J. Laurmann), Academic Press, New York, p. 45.

5. Cox, R. N.: 1963, Rarefied Gas Dynamics, vol. II, (ed. by J. Laurmann), Academic Press, New York, p. 1.

6. Bienkowski, G. K.: 1966, A.I.A.A. Preprint paper No. 66-6.

7. Moskalenko, A. M.: 1964, Geomagnetizm i Aeronomiya 4, 798.

8. Quasi-Neutral Flows

1. Gurevich, A. V., Pitayevsky, L. P., and Pariyskaya, L. V.: 1966, Soviet Phys. JETP 22, 449

2. Gurevich, A. V. and Pitayevsky, L. P.: 1964, Geomagnetizm i Aeronomiya 4, 687.

3. Al'pert Ya. L., Gurevich, A. V., and Pitayevsky, L. P.: 1965, Space Physics with Artificial Satellites, Consultant Bureau, New York.

4. Panchenko, Yu. M. and Pitayevsky, L. P.: 1964, Geomagnetizm i Aeronomiya 4, 199.

9. Near-Wake Flows

1. Davis, A. H. and Harris, I. : 1961, Rarefied Gas Dynamics (ed. by L. Talbot), Academic Press, New York, p. 691.

2. Sawchuk, W.: 1963, Rarefied Gas Dynamics (ed. by J. Laurmann), Academic Press, New York, p. 34.

3. Maslennikov, M. V. and Sigov, Yu. S.: 1967, Rarefied Gas Dynamics, vol. II, (ed. by C. L. Brundin), Academic Press, New York, p. 1657.

4. Clayden, W. A. and Hurdle, C. V.: 1967, Rarefied Gas Dynamics, vol. II, (ed. by C. L. Brundin), Academic Press, New York, p. 1717.

5. Taylor, J. C.: 1967, Planetary Space Sci. 15, 155, 467.

6. Prager, D. J. and Rasmussen, M. L.: 1967, SUDAAR Rept. No. 299 Stanford University.

7. Shea, J. J.: 1967, Rarefied Gas Dynamics, vol. II, (ed. by C. L. Brundin), p. 1671.

8. Moskalenko, A. M.: 1964, Geomagnetizm i Aeronomiya 4, 203, 404.

10. Far-Wake Flows

1. Dolph, C. L. and Weil, H.: 1961, Electromagnetic Effects of Re-entry, Pergamon Press, London, p. 123.

2. Kraus, L. and Watson, K. M.: 1958, Phys. Fluids 1, 480.

3. Rand, S.: 1960, Phys. Fluids 3, 588.

4. Yoshihara, H.: 1961, Phys. Fluids 4, 588.

5. Rand, S.: 1960, Phys. Fluids 3, 265.

6. Vaglio-Laurin, R. and Pan, Y. S.: 1967, Rarefied Gas Dynamics, vol. II, (ed. by C. L. Brundin), Academic Press, New York, p. 1687. 
7. Ryhming, I. L.: 1961, Magnetohydrodynamics (ed. by A. B. Cambel), Northwestern University Press, Evanston, Ill., p. 381.

8. Vas'kov, V. V.: 1966, Soviet Phys. JETP 23, 748.

9. Pitayevsky, L. P.: 1963, Soviet Phys. JETP 17, 658.

10. Al'pert, Ya. L.: 1961, Soviet Phys. USPEKHI 4, 479.

\section{Plasma Resonances (Alouette and Ariel Satellites)}

1. Bernstein, I. B.: 1958, Phys. Rev. 109, 10.

2. Stix, T. H.: 1962, The Theory of Plasma Waves, McGraw-Hill, New York.

3. Bernstein, I. B., Trehan, S. K., and Weenink, M. P. H.: 1964, Nucl. Fusion 4, 61.

4. Calvert, W. and Goe, G. B.: 1963, J. Geophys. Res. 68, 6113.

5. Fried, B. D. and Conte, S. D.: 1961, The Plasma Dispersion Function, Academic Press, New York.

6. Ginzburg, V. L.: 1961, Propagation of Electromagnetic Waves in Plasma, Gordon and Breach, New York.

7. Johnston, T. W. and Nuhall, J.: 1964, J, Geophys. Res. 69, 2305.

8. Knecht, R. W. and Russell, S.: 1962, J. Geophys. Res. 67, 1178.

9. Knecht, R. W., Van Fandt, T. E., and Russell, S.: 1961, J. Geophys. Res. 66, 3078.

10. Lockwood, G. E. K.: 1963, Can. J. Phys, 41, 190.

11. Calvert, W. and Van Fandt, T. E.: 1966, J. Geophys. Res. 71, 1799.

12. Nuttall, J.: 1965, J. Geophys. Res. 70, 1119.

13. Wallis, G.: 1965, J. Geophys. Res. 70, 1113.

14. Crawford, F. W.: 1965, Nucl. Fusion 5, 73.

15. Deering, W. D. and Fejer, J. A.: 1965, Phys. Fluids 8, 2066.

16. Harp, R. S.: 1965, Appl. Phys, Letters 6, 51.

17. Shkarofsky, I. P. and Johnston, T. W.: 1965, Phys. Rev. Letters 15, 51.

18. Farley, D. T., Dougherty, J. P., and Barron, D. W.: 1961, Proc. Roy. Soc. (London) A263, 238.

19. Kuehl, H. H.: 1962, Phys. Fluids 5, 1095.

20. Kuehl, H. H.: 1963, Phys. Fluids 6, 1465.

\section{Meteor - Radio Echo}

1. Millman, P. M. and McKinley, D. W. R.: 1963, The Moon, Meteorites, and Comets (ed. by B. M. Middlehurst and G. P. Kuiper), University of Chicago Press, Chicago, ch. 1.

2. Jacchia, L. G.: 1963, The Moon, Meteorites, and Comets (ed. by B. M. Middlehurst and G. P. Kuiper), University of Chicago Press, Chicago, ch. 22.

3. Greenhow, J. S.: 1963, Smithsonian Contrib. Astrophys. 7, 5, Smithsonian Institution, Washington, D. C.

4. Kashcheyev, B. L. and Lebedinets, V. N.: 1963, Smithsonian Contrib. Astrophys., 7, 19, Smithsonian Institution, Washington, D.C.

5. Weiss, A. A.: 1963, Smithsonian Contrib. Astrophys. 7, 41, Smithsonian Institution, Washington, D.C.

6. Dubin, M., Alexander, W. M., and Berg, O. E.: 1963, Smithsonian Contrib. Astrophys, 7, 109, Smithsonian Institution, Washington, D.C.

7. Francey, J. L. A.: 1963, Australian J. Phys. 16, 500.

8. Kalinin, Yu. K.: 1965, Geomagnetizm i Aeronomiya 5.

9. Kalinin, Yu. K.: 1963, Geomagnetizm i Aeronomiya 3, 779.

10. Lebedinets, V. N. and Portnyagin, Yu. I.: 1966, Geomagnetizm i Aeronomiya 4.

11. Dokuchaev, V. P.: 1960, Soviet Astron. A. J. 4, 106.

12. Furman, A. M.: 1964, Soviet Astron. A. J. 7, 559.

13. Furman, A. M.: 1967, Soviet Astron. A. J. 10, 844.

14. Dubin, M.: 1955, Meteors (ed. by T. R. Kaiser), Pergamon Press, London, p. 29.

15. Moiseevich, B. L.: 1955, Meteors (ed. by T. R. Kaiser), Pergamon Press, London, p. 23.

16. Sida, D. W.: 1955, Meteors (ed. by T. R. Kaiser), Pergamon Press, London, p. 26.

17. Evans, S. and Hall, Y. E.: 1955, Meteors (ed. by T. R. Kaiser), Pergamon Press, London, p. 18.

18. Massey, H. S. W. and Sida, D. W.: 1955, Phil. Mag. 46, 190.

19. Herlofson, N.: 1948, Rept. Prog. Phys. 11, 444.

20. Kaiser, T. R. and Closs, R. L.: 1952, Phil. Mag. 43, 1.

21. Sandler, S. I. and Dahler, J. S.: 1964, Phys. Fluids 7, 1743. 
13. Comet

1. Biermann, L.: 1951, Z. Astrophys. 29, 274.

2. Biermann, L.: 1952, Z. Naturforsch. 8a, 127.

3. Biermann, L.: 1960, Rev. Mod. Phys. 32, 1008.

4. Marochnik, L. S.: 1964, Soviet Phys. USPEKHI 7, 80.

5. Whipple, F. L.: 1950, Astrophys. J. 111, 375.

6. Whipple, F. L.: 1951, Astrophys. J. 113, 464.

7. Roemer, E.: 1963, The Moon, Meteorites, and Comets (ed. by B. M. Middlehurst and G. P. Kuiper), University of Chicago Press, Chicago, ch. 15.

8. Wurm, K.: 1963, The Moon, Meteorites, and Comets (ed. by B. M. Middlehurst and G. P. Kuiper), University of Chicago Press, Chicago, ch. 17.

9. Biermann, L. and Lüst, R.: 1963, The Moon, Meteorites, and Comets (ed. by B. M. Middlehurst and G. P. Kuiper), University of Chicago Press, Chicago, ch. 18.

10. Whipple, F. L.: 1963, The Moon, Meteorites, and Comets (ed. by B. M. Middlehurst and G. P. Kuiper), University of Chicago Press, Chicago, ch. 19.

11. Oort, J. H.: 1963, The Moon, Meteorites, and Comets (ed. by B. M. Middlehurst and G. P. Kuiper), University of Chicago Press, Chicago, ch. 20.

12. Biermann, L. and Trefftz, E.: 1960, Z. Astrophys. 49, 111.

13. Blackwell, D. E. and Ingham, M. F.: 1961, Monthly Notices Roy. Astron. Soc. 122, 113, 129, 143.

14. Harwit, M. and Hoyle, F.: 1962, Astrophys. J. 135, 867, 875.

15. Finson, M. L. and Probstein, F. R.: 1968, Astrophys. J. 154, 327, 353. 